\title{
Biodegradable Polymers for Microencapsulation Systems
}

\author{
Joana Filipa Parente $(\mathbb{D}$, Vânia Isabel Sousa $(\mathbb{D}$, Juliana Filipa Marques $(\mathbb{D}$, \\ Marta Adriana Forte $\mathbb{D}$, and Carlos José Tavares $\mathbb{D}$ \\ Centre of Physics of the Minho and Porto Universities (CF-UM-UP), University of Minho, Campus of Azurém, \\ 4835-386 Guimarães, Portugal
}

Correspondence should be addressed to Carlos José Tavares; ctavares@fisica.uminho.pt

Received 12 August 2021; Revised 3 January 2022; Accepted 27 January 2022; Published 14 February 2022

Academic Editor: Bingang Xu

\begin{abstract}
Copyright (C) 2022 Joana Filipa Parente et al. This is an open access article distributed under the Creative Commons Attribution License, which permits unrestricted use, distribution, and reproduction in any medium, provided the original work is properly cited.
\end{abstract}

\begin{abstract}
Environmentally friendly alternatives have become sought after upon the development of scientific research and industrial processes. Recent trends suggest biodegradable polymers as the most promising solution for synthetic microcapsule systems. Safety, efficiency, biocompatibility, and biodegradability are some of the properties that biodegradable systems in microencapsulation can provide for a broad spectrum of applications. The controlled release of encapsulated active agents is a research field that, over the years, has been constantly innovating due to the promising applications in the areas of pharmaceutical, cosmetic, textile industry, among others. This article presents an overview of different polymers with potential for microcapsule synthesis, namely, biodegradable polymers. First, natural polymers are discussed, which are divided into two categories: polysaccharide-based polymers (cellulose, starch, chitosan, and alginate) and protein polymers (gelatin). Second, synthetic polymers are described, where biodegradable polymers such as polyesters, polyamides, among others appear as examples. For each polymer, this review presents its origin, relevant properties, applications, and examples found in the literature regarding its use in biodegradable microencapsulation systems.
\end{abstract}

\section{Introduction}

Over the years, polymers have undergone several advances in the improvement of their synthesis and biodegradability [1]. These materials have attracted several researches worldwide in therapeutic administration, in particular on the administration of drugs and in biomedical field applications [2, 3]. However, many problems have arisen because some polymers are not biodegradable. Some nondegradable polymers have several disadvantages, as the prominent toxicity. This disadvantage has concerned several researchers to circumvent this problem; thus, the emergence of biodegradable polymers. Biodegradable polymeric matrices have been developed for sustained release, controlled release, and delayed release [4].

Biodegradation is a natural process where organic chemicals in the environment are converted into simpler compounds, mineralized, and redistributed by elementary cycles. Biodegradable polymers undergo biodegradation in vivo, enzymatic, or nonenzymatic, and produce biocompatible or harmless by-products [3].

Since the majority of biodegradable polymers are not harmful to the body or the environment, they have achieved a remarkable focus for new chemical macromolecules scheme medication administration [5], revealing the absence of inflammatory effects, good permeability, and inhibition of toxic degradation products [6]. In addition to the pharmaceutical industry, these biodegradable polymers have important applications in medicine, agriculture, and packaging.

The application of biodegradable polymers in these different areas is carried out mainly through microparticles, such as microcapsules and microspheres (1-1000 $\mu \mathrm{m}$ in diameter). These small particles are made up of an active agent surrounded by a synthetic polymeric shell, which protects the internal part from the external environment [7]. The encapsulated substances are isolated from various conditions such as light, oxygen, heat, humidity, and interactions with other substances. Furthermore, the microencapsulation technique is 
widely used for the controlled release of the active agent and to control the release of volatile substances. The encapsulated substance can be released through several mechanisms that involve specific conditions such as $\mathrm{pH}$, temperature, diffusion, vapour pressure, and mechanical actions [8].

Microencapsulation is a technology that has gained prominence over time. Currently, it is used in several industrial areas, mainly in pharmaceutical, medical, food, textile, and cosmetics industries [9]. Several scientific studies demonstrate a growing interest of the scientific community in the microencapsulation of active agents using biodegradable and biocompatible polymeric materials due to extensive search for environmentally and human health-friendly formulations [10].

This review reports the origin of biodegradable polymers, being divided into two categories, natural polymers, and synthetic polymers (Figure 1). Several characteristics and applications of these materials are approached, as well as a review of the most relevant scientific works in their applicability in microencapsulation technology.

\section{Biodegradable Polymers Used for Microencapsulation}

2.1. Natural Polymers. Natural biodegradable polymers are formed in nature during the growth cycles of organisms. These polymers have several advantages, such as ease to obtain, biocompatibility, and biodegradability. Natural polymers are planted and animal-based and most have solubility in water. As main properties, it can be highlighted their capacity to emulsify oils, stabilize complex formulas, and prolong the effectiveness of active agents. Polymerization reactions in growth chains catalysed by enzymes are present in the synthesis of these polymers. Natural polymers are versatile for controlled drug delivery, gene delivery, regenerative medicine, and other biomedical applications. Natural polymers show less toxicities when compared with synthetic polymers [12].

2.1.1. Polysaccharide-Based Polymers. Polysaccharides are carbohydrates made up of units of monosaccharides in their backbone and are usually joined by O-glycosidic bonds of the ether bond. They are biodegradable, very stable, biocompatible, nontoxic, hydrophilic, and easily modified. Regarding the application, polysaccharides are used in the administration and targeting of drugs and the most used are alginate, chitosan, among others [13, 14]. For material applications, the main polysaccharides of interest are cellulose and starch.

(1) Starch. Starch (Figure 2) is a white, monotonous, and odourless powder, insoluble in cold water or alcohol. This polymer is widely found in plants, being the most common carbohydrate in a variety of human food, such as potatoes, rice, and wheat.

Amylose is the linear constituent of starch, which was used to make mixed films of amylose and ethyl cellulose as coatings [15]. It has also been applied for the delivery of antiarthritic drugs and for the delivery of pesticides [16].

Probiotics are living microorganisms that have been administered to consumers in adequate amounts. Resistant starch (RS) is a prebiotic substance that provides a beneficial physiological effect on the host by stimulating the growth or favourable activity of a limited number of bacteria [17]. Foods that contain both probiotics and prebiotics are referred to as symbiotic or functional foods [18]. In the colon, prebiotic compounds are consumed by probiotics as an energy source, increasing the number of cells, while pathogenic microorganisms decrease [19]. In this particular case, microencapsulation can be applied to protect probiotic foods and microorganisms during processing, storage, and transition in the acidic environment of the stomach [20-22]. The effects of RS on encapsulation materials have been studied and are described in Figure 3. A certain combination of encapsulation materials, such as alginate and whey protein with RS, can be used to improve the tolerance of probiotics and microcapsules against digestive stresses [22-24]. These microcapsules maintain greater viability of microorganisms and do not show side effects on sensory characteristics during storage periods $[20,22]$. Resistant starch has been studied to improve clinical conditions related to inflammatory bowel diseases, immunostimulatory activities, and to protect against colon cancer [25]. There are other studies on the encapsulation of nutraceutical components, such as folic acid, with RS-biofilm combinations that support the data obtained with living organisms [26].

In 2013, Ying et al. compared the survival of spray-dried microencapsulated Lactobacillus rhamnosus GG (LGG) added to apple juice or citrate buffer ( $\mathrm{pH} 3.5)$ [24]. The LGG was encapsulated through three different matrices:

(i) Whey protein isolate (WPI) alone

(ii) WPI in combination with physically modified resistant starch (RS) in different proportions $(4: 1,1: 1$, and $1: 4)$

(iii) RS alone

The authors found that LGG microcapsules containing WPI alone or WPI in combination with RS provided better protection to LGG in apple juice or citrate buffer, compared to the formulation containing RS alone. This fact can be explained by the ability of WPI to create a buffered microenvironment within the hydrated colloid particle around the incorporated LGG. Through confocal laser scanning microscopy (CLSM) technique, it was verified that WPI and the physically modified RS can form a gel matrix when using LGG microcapsules dispersed in apple juice for 24 hours (Figure 4). Most of LGG was trapped within the encapsulation matrix after 24 hours.

Li et al. prepared starch microcapsules using a premixed membrane emulsification method [27]. To manufacture microcapsules with a uniform and controllable size, the authors optimized the conditions of the process. A water insoluble pesticide-controlled release system was developed using avermectin (Av) as active agent. Scanning electron 


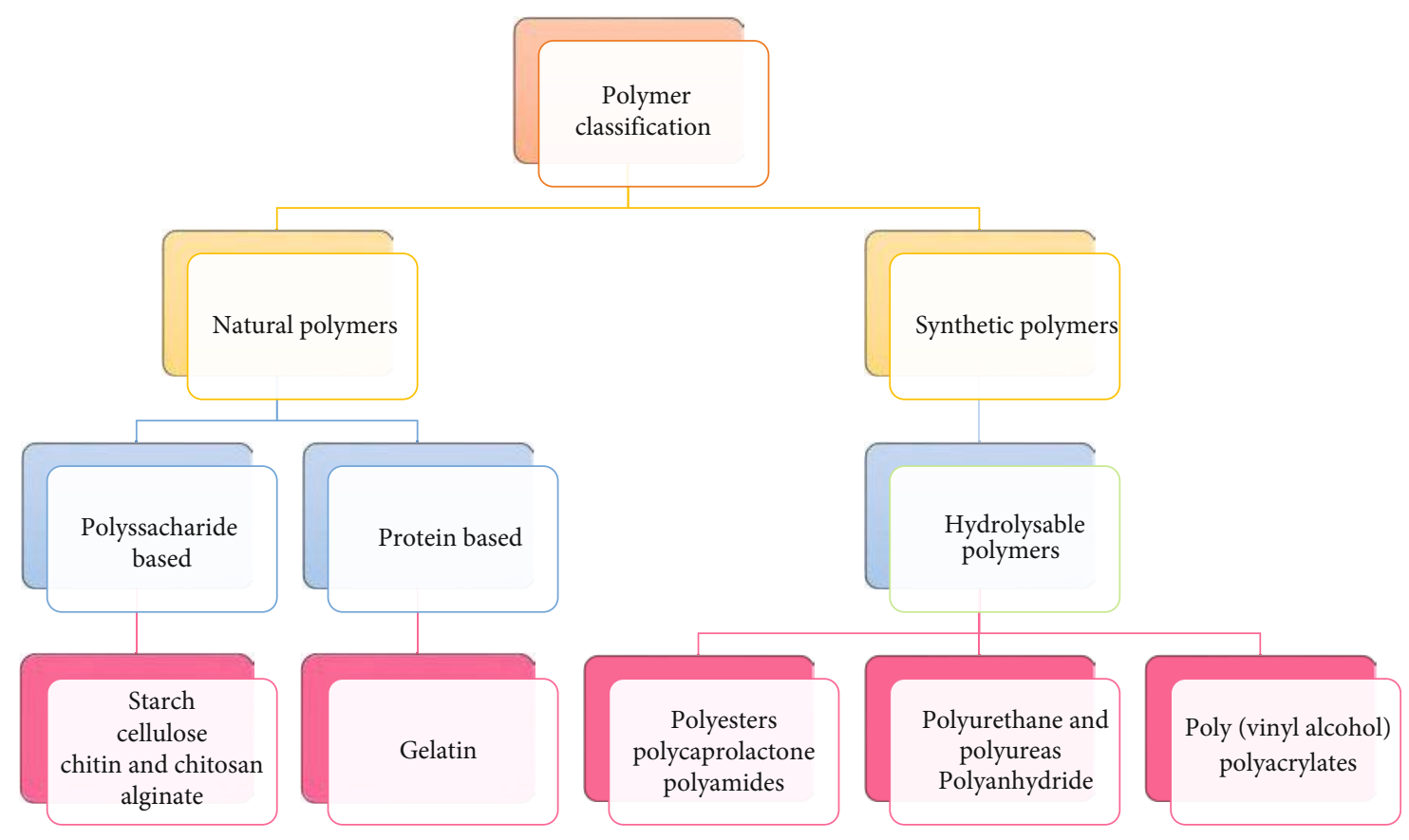

Figure 1: Polymer classification [11].<smiles>OCC1O[C@H](O)[C@H](O)C(O)[C@@H]1O[C@H]1OC(CO)[C@H](O)[C@@H](O)[C@H]1O</smiles>

Figure 2: Chemical structure of starch.

microscopy (SEM) observations showed that the resultant microcapsules were uniform when dichloromethane (DCM) was used as an oil phase, and a stable emulsion was produced (Figure 5(a)). When ethyl acetate (EA) was used to replace pure water, a milky emulsion was obtained, and the microcapsules presented a more heterogeneous size distribution (Figure 5(b)). With these results, it was concluded that DCM was preferred to make the starch microcapsules with a better size control.

Etchepare et al. used resistant starch (Hi-maize) in the microencapsulation of Lactobacillus acidophilus on alginate beads, in a simulated digestive system, and under different storage temperatures [23]. These authors found that $\mathrm{Hi}$ maize provided better protection for probiotics after exposure to simulated gastrointestinal juice, for wet and lyophilized microcapsules. Through optical microscopy (OM), it was found that the wet microcapsules were spherical with the core material distributed throughout the matrix (Figure 6). Once the active material was located inside the particle, alginate particles and microorganisms were observed inside the microparticle, showing that the microorganism microencapsulation was effective in both treatments.

Cai et al. developed carboxymethyl starch (CMS)/ xanthan gum (XG) combination systems to encapsulate blueberry anthocyanins (ANS) and improve their stability [28]. The study of the physical and chemical properties, thermal stability, and storage stability of the system allowed to conclude that the microcapsules showed high encapsulation efficiency and thermal stability. ANS stability was increased to $76.11 \%$ after 30 days storage $\left(37^{\circ} \mathrm{C}\right)$. In addition to being a low-cost material, it was concluded that CMS/XG, as a microcapsule wall material, can protect blueberry anthocyanin. Moreover, in vitro release experiments of anthocyanins in microcapsules were performed under simulated gastric and intestinal fluid. These studies showed that ANS were retained within the CMS/XG-ANS microcapsules in the stomach and released in the intestine proving that the CMS/XG combination improves the stability of ANS and promotes target distribution in the intestine (Figure 7).

There are only a few studies on the preparation and stability of CMS/XG-ANS microcapsules. Hence, this field requires additional research and development, such as more in vivo analysis, to guarantee the improvement of the stability of blueberry anthocyanins and the correct target distribution in the intestine.

(2) Cellulose. Many researchers defend that polymer chemistry started with the characterization of cellulose. Cellulose is a long-chain polymer composed of a single monomer (glucose) and can be classified as a polysaccharide (Figure 8). Cellulose is one of the main constituents of plant cell walls, being a highly crystalline polymer with high molecular weight.

Cellulose presents different properties according to the surrounding environment, particularly in the aerobic environment of soil, which usually contains pools of several types of bacteria and fungi that cooperate. Primary 


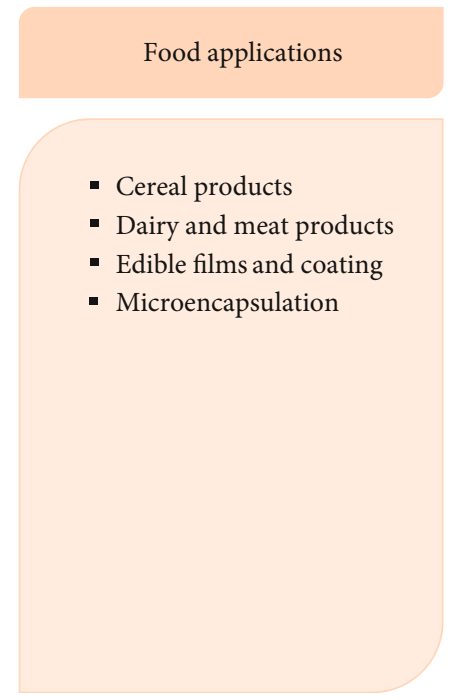

Effects on product properties

- Inert fiber source

- Stable structure in storage period

- Increase in loaf volume of gluten-free breads, and pasta firmness

- Water structuring and entrapping ability in dairy products

- Compact and hydrophobic microcapsule surface

- Stronger film form and good process ability

FigURE 3: Resistant starch applications and quality effects in some food products.

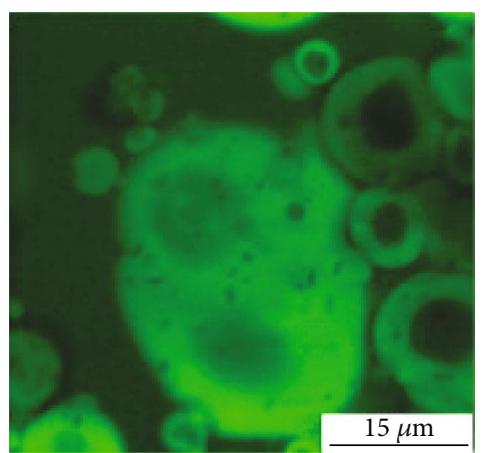

(a)

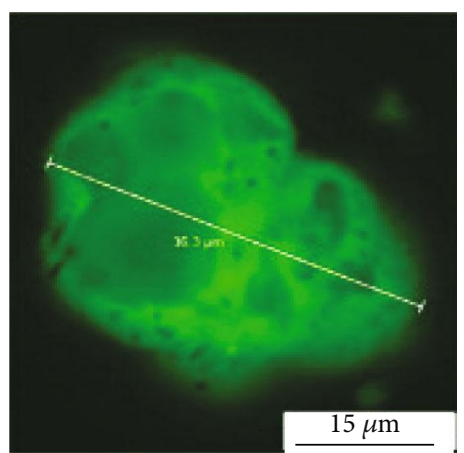

(b)

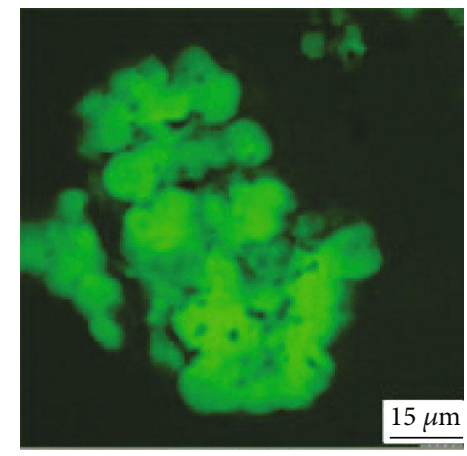

(c)

FIGURE 4: Confocal laser scanning microscopy micrographs showing the integrity of microencapsulated LGG formulations containing (a) WPI, (b) 1WPI:1RS, and (c) RS dispersed in apple juice. The micrographs were taken 24 hours after dispersion of the microencapsulated LGG [24].

microorganisms break down cellulose into glucose and cellodextrins, and secondary microorganisms provide enzymes that break down cellodextrins into glucose. The consumption of glucose supports the growth of primary microorganism and prevents the accumulation of cellodextrins that can inhibit glucanases. Carbon dioxide and water are the final products of this process. In anaerobic environment, a varied number of final products are formed, in which carbon diox- ide can be produced by oxidative reactions using inorganic compounds. The hydrogen produced by some anaerobic bacteria can be used by autotrophic bacteria to reduce oxidized compounds.

Another class of polymers that can be used is cellulose esters because of its potential to participate in the carbon cycle. Cellulose acetate is used in applications, ranging from fibers to films and thermoplastics for injection molding. This 


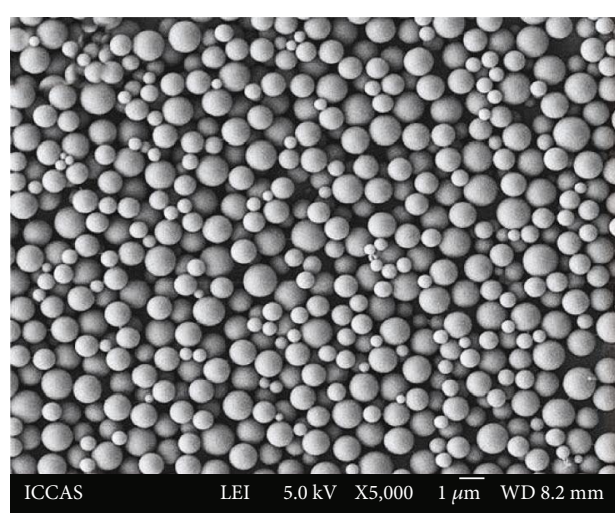

(a)

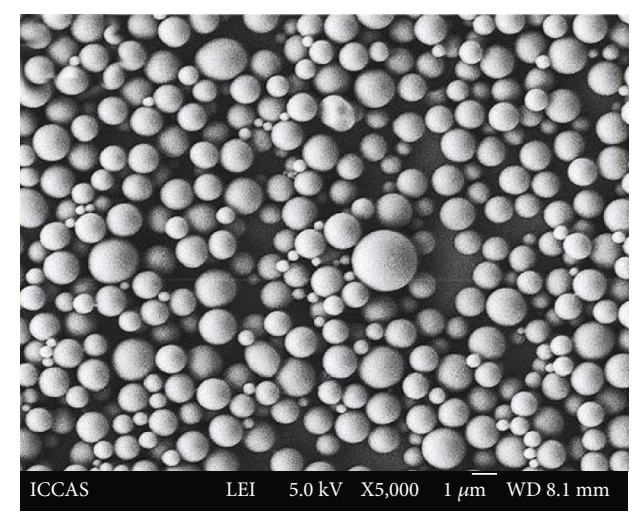

(b)

FIGURE 5: SEM micrographs of starch microcapsules prepared with (a) DCM and (b) EA [27].

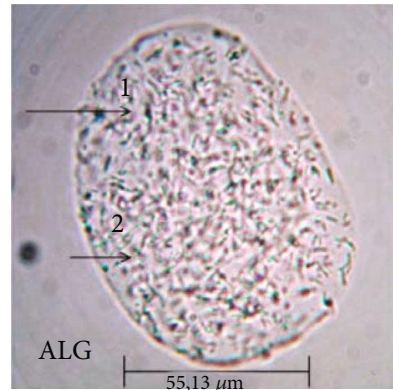

(a)

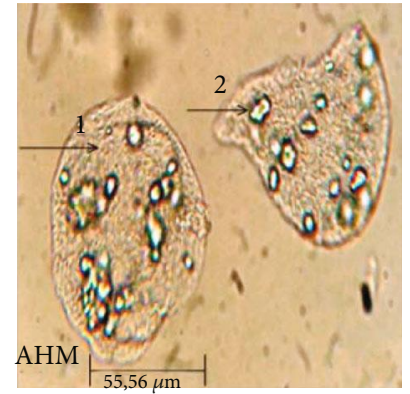

(b)

FIgURE 6: Optical micrographs of both alginate and alginate $+\mathrm{Hi}-$ maize (AHM) microparticles (a) alginate microparticle. Label 1 indicates sodium alginate inside the particle, and label 2 indicates the microorganism within the particle (100× magnification); (b) AHM microparticles, in which label 1 shows the sodium alginate in the interior of the particle, and label 2 shows the prebiotic Hi-maize (60×). Pictures are representative of triplicates [23].

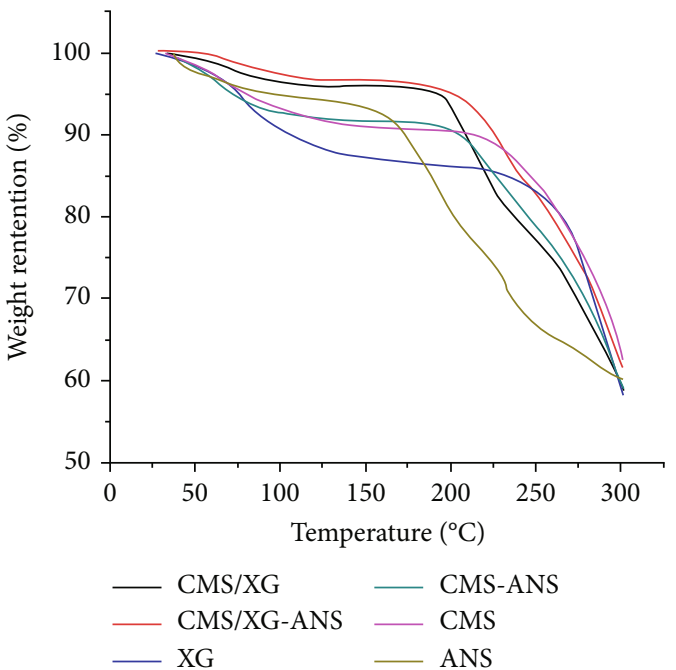

(a)

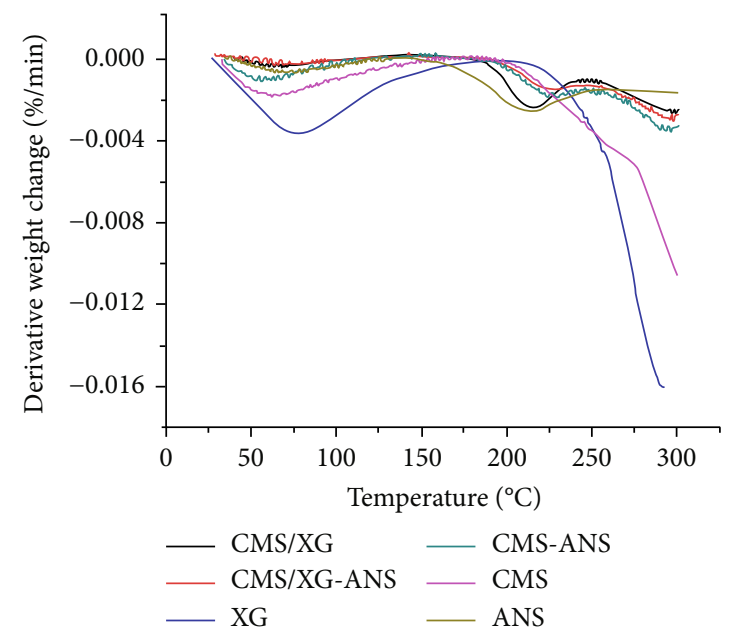

(b)

FIgURe 7: In vitro release kinetics of anthocyanins from (a) CMS-ANS, (b) CMS/XG(150/1)-ANS, (c) CMS/XG(120/1)-ANS, (d) CMS/ XG(90/1)-ANS, (e) CMS/XG(60/1)-ANS, and (f) CMS/XG(30/1)-ANS under stimulated gastric (a) and intestinal (b) conditions [28].

material has relatively low physical properties and material costs, which tend to exclude other biodegradable polymers from being widely accepted on the market.
Tangsongcharoen et al. studied the preparation of cellulose-based microcapsules having as active agent gallic acid (GA), which is an important antioxidant of the 


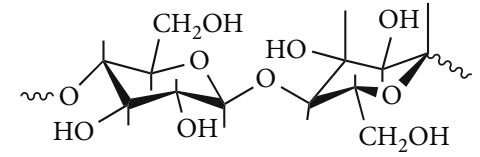

Figure 8: Chemical structure of cellulose.

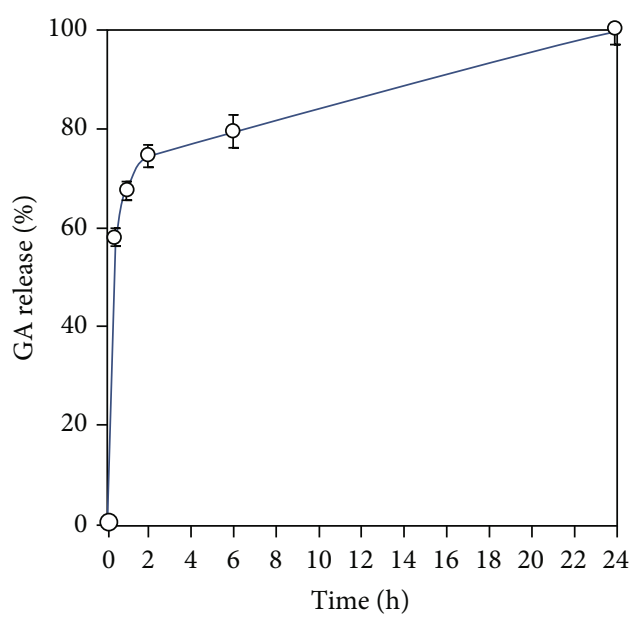

FIGURE 9: In vitro release of the GA encapsulated within m-CMCg-PMAA microcapsules in PBS of pH 5.5 [29].

bambara groundnut extract [29]. GA was used as a core material, and carboxymethylcellulose (CMC) was used as a shell. CMC was modified with 3-(trimethoxysilyl)propyl methacrylate (MPS) as a silane coupling agent in order to obtain a high encapsulation efficiency. It was further polymerized with methacrylic acid (MAA) monomer forming a biocompatible polymer shell with grafted PMAA-CMC (mCMC-g-PMAA). After polymerization, spherical microcapsules of m-CMC- $g$-PMAA encapsulating GA were formed. An enhancement of the encapsulation efficiency was observed with the increase of MAA content. This increase could be due to the increased hydrophilicity of the aqueous phase. Regarding the rapid release and noncytotoxic characteristics, it was found that microcapsules with a weight ratio m-CMC:MAA of 33:67 showed greater encapsulation efficiency (Figure 9), thus, being suitable for use in cosmetic products.

Chen et al. developed an ecological approach to multifunctional water-based fabric coatings using ethyl cellulose/ silica hybrid microcapsules [30]. Through a diffusion of solvent in emulsion, ethyl cellulose/silica hybrid microcapsules were obtained, having encapsulated lavender fragrance oil (LFO). These microcapsules have a $30 \mu \mathrm{m}$ diameter coreshell structure, UV absorbers, and silica grafted with methacrylic acid into the shell. These hybrid microcapsules were posteriorly added to water-based polysiloxane resins to form multifunctional fabric coatings. Through Figure 10, it can be seen that the residual amount of lavender fragrance oil (LFO) in the fabric treated with pure LFO is still high at the beginning. However, the residual rate of LFO decreased markedly after five days, while the LFO in the fabrics disappeared after fifteen days. Compared to fabrics treated with pure LFO, fabrics treated with control microcapsules with lav- ender fragrance oil (LFO-CM) and fabrics treated with cellulose/silica hybrid microcapsules with lavender fragrance oil (LFO-CSHM) can sustain fragrances for a long-time, with the residual rate of LFO in the LFO-CSHM treated fabrics remained above $30 \%$ after 90 days. This fact is due to the slow release of the lavender fragrance oil in the capsules. The application of this fabric coating can be used on sportswear, curtains, and other outdoor fabrics.

Simões et al. used the solvent evaporation method to produce microspheres loaded with eugenol using different cellulose derivatives (ethyl cellulose (EC), cellulose acetate (CA), cellulose acetate butyrate (CAB), and cellulose acetate phthalate (CAP)), which were later incorporated into the textile industry [31]. Through various characterization methods, the resulting microspheres were evaluated for their shape, size, temperature stability, encapsulation efficiency, and kinetic release of eugenol. The obtained results suggest that microspheres based on AC performed better, thus leading to microspheres loaded with eugenol to be successfully incorporated into cotton fabrics. It was concluded that these microspheres showed promising results for the retention and protection of hydrophobic active compounds for possible textile applications.

Simões et al. used SEM to study the eugenol-loaded microspheres morphology, and the produced micrographs clearly show that CA and CAB microspheres (Figures 11(a) and $11(\mathrm{~b})$ ) are smaller in relation to the other two samples (CAP and EC). Furthermore, it can be observed that microspheres of CA are slightly smaller compared to microspheres of $\mathrm{CAB}$, proving to be quite homogeneous in size and shape. In the case of the microspheres of EC and CAP, these exhibit a much larger size, with a wider size distribution (Figures 11(c) and 11(d)). Through this characterization, it was verified that the produced microcapsules are spherical with smooth surfaces, except for particles based on EC that present surface irregularities (pores and/or holes), in addition to less sphericity (Figure 11(d)).

The control release ability of cellulose-derived microspheres was assessed using eugenol release profiles. As shown in Figure 12, EC-based microspheres are the ones with the fastest eugenol release rate, followed by CAP, $\mathrm{CAB}$, and $\mathrm{CA}$ microspheres. A result that proved to be interesting is that the maximum percentage of eugenol release is dependent on the cellulose derivative used in the microencapsulation, and it can vary, over 63 days, from 35\% for the CA microspheres to $80 \%$ for the case of the EC microspheres.

Comparing encapsulation efficiency values in Table 1 with release profiles in Figure 12, it can be concluded that, although EC-based microcapsules retain less eugenol, this material exhibits a faster release rate, which is probably favored by the surface porosity (Figure 11(d)). However, these EC microspheres and CAP microspheres were excluded due to their size and release capacity. Through the analysis of the obtained results, CA microspheres reveal a greater encapsulation efficiency and a slower eugenol release profile (Table 1). To summarize the results described above, CA microspheres incorporating eugenol proved not only to have a uniform size distribution but also the highest 


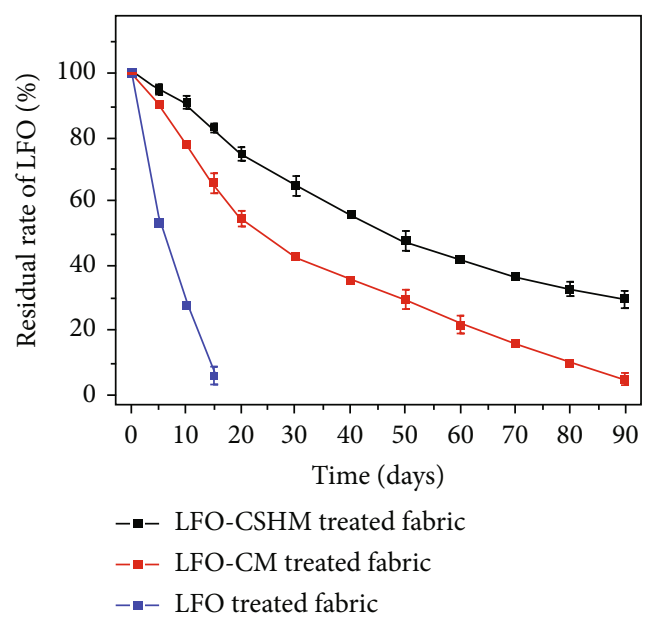

(a)

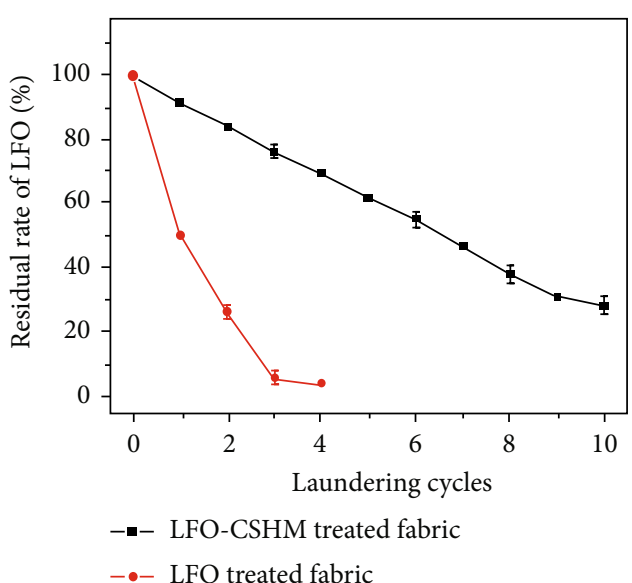

(b)

FIGURE 10: Control release of (a) LFO from LFO-treated, LFO-CM-treated (40\%LFO - CM + polysiloxane resins) and LFO-CSHM-treated (40\%LFO - CSHM + polysiloxane resins) cotton fabrics. (b) LFO from LFO-treated (40\%LFO - CM + polysiloxane resins) and LFO-CSHMtreated (40\%LFO - CSHM + polysiloxane resins) cotton fabrics after repeated laundering [30].

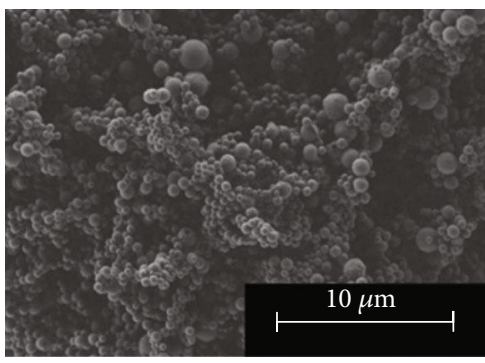

(a)

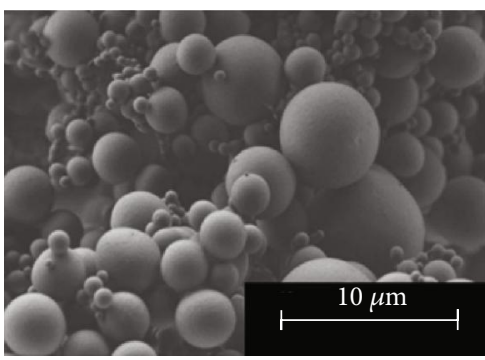

(c)

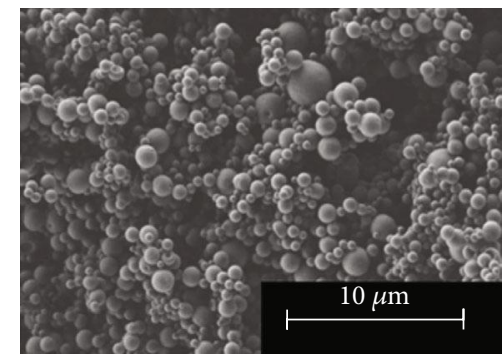

(b)

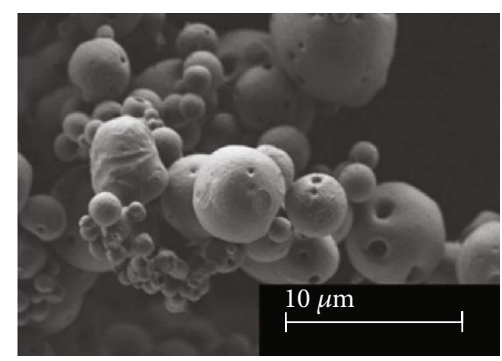

(d)

FIGURE 11: FE-SEM images of the eugenol-loaded CA (a), CAB (b), CAP (c), and EC (d) microspheres produced at smaller scale (1500× magnification) [31].

encapsulation efficiency, as well as the best controlled release capacity.

Cellulose acetate is a cellulose derivative suitable for microencapsulation of essential oils, through simple methods such as solvent evaporation. In our opinion, this study shows promising results in the retention and protection of eugenol and its subsequent textile application. The work carried by Simões et al. is quite complete, encompassing the evaluation of different production volumes (100$2000 \mathrm{~mL}$ ), through various parameters, such as particle size, surface charge, encapsulation efficiency, and performance in the release of active compound. In addition, the authors successfully impregnated cellulose acetate microspheres in tex- tile substrates, proving that the developed microspheres are suitable for textile functionalization.

(3) Chitin and Chitosan. Chitin (Figure 13) is a macromolecule found in several types of seafood, such as crabs, lobsters, shrimp, and insects. It is insoluble in its native form, but soluble in water in the partially deacetylated form. By using chitin and chitosan as carriers, it was possible to synthesize a water-soluble prodrug [32].

A study on the use of chitin in absorbable sutures showed that chitins have the least elongation among the suture materials made up of chitin, poly(glycolic acid) 


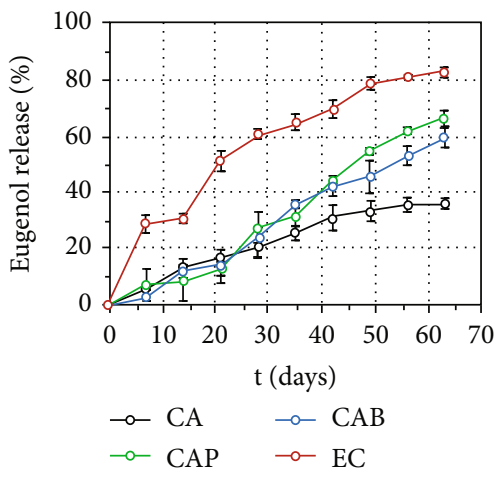

FIgURE 12: Eugenol release profiles of a CA, CAB, CAP, and EC eugenol-loaded microspheres [31].

(PGA), among others [33]. Its derivatives can also be used as drug carriers [34].

Through the deacetylation of chitin (Figure 14), a cationic polysaccharide is produced, named chitosan, whose chemical structure is presented in Figure 15.

Chitosan has excellent properties, which facilitate interaction with the human body, revealing a variety of applications in the field of biomaterials [35]. It has also been tested for applications such as contact lenses, tissue adhesion, and prevention of bacterial adhesion, sutures, and others [36]. Chitosan and chitin have been used in the treatment of wounds, ulcers, and burns due to its accelerating effect of wound healing. Due to its affinity and cellular biodegradability, it has been applied in tissue regeneration and restoration, including its use in perspective as a structural material in tissue engineering.

Regarding its sensitivity to $\mathrm{pH}$, a drug delivery system consisting of a hybrid network of chitosan/gelatin crosslinked with glutaraldehyde was developed [37]. Chitosan/ xanthan microspheres have also been prepared to confer a protective effect to the medication in the gastric and intestinal parts of body [38]. In another work, chitosan/carboxymethyl cellulose (CH/CMC) microcapsules of different compositions were prepared and tested as a protective matrix against the acidic $\mathrm{pH}$ of the stomach, for oral administration of proteins and drugs [39].

Another field where chitin and chitosan can be used is in agriculture. This application has followed four main directions, namely,

(a) Protection of plants against plagues and diseases during preharvest and postharvest

(b) Promoting of antagonist microorganism action and biological control

(c) Support of beneficial plant-microorganism symbiotic relationships

(d) Plant growth regulation and development

Chitin and its derivatives have been used to induce defensive mechanisms in plants, providing protection against many plant diseases [40]. Chitin and chitosan have fungicidal activity against many phytopathogenic fungi, and chitosan also has antiviral and antibacterial activity [41]. In one case, chitin was added to the soil in order to destroy the eggs and cuticles of young nematodes that have chitin in their composition, resulting from the induced increase in the population of microorganisms [42]. It has been studied that covering fruits and vegetables with a chitosan film gives an antimicrobial protection and a longer shelf life [43].

By using an oil-in-water (o/w) emulsion, Hsieh et al. prepared chitosan microcapsules for the encapsulation of citronella oil, where it was verified the condition of microcapsule's formation and the influence for controlled release effect of citronella oil, through the application of thermal pretreatment [44]. From the study of microcapsule's formation, it was realized that changes in concentration of chitosan affect the efficiency of citronella oil encapsulation. Furthermore, when concentrations of chitosan are $0.5 \%$, $1.0 \%$, and $1.5 \%$, the efficiencies of encapsulation are $98.2 \%$, $95.8 \%$, and $94.7 \%$, respectively. As the emulsification agitation speed is increased, the size of chitosan microcapsules decreases.

In Figure 16, it can be observed chitosan microcapsules with concentrations of $0.5 \mathrm{wt} \%, 1 \mathrm{wt} \%$, and $1.5 \mathrm{wt} \%$ and their ability to release encapsulated citronella oil. From this figure, it can be seen that the release of encapsulated citronella oil is slower than non-encapsulated citronella oil. Additionally, for a higher concentration of chitosan, a slower release of oil is registered. This fact can be explained because the higher the concentration of chitosan, the thicker is the microcapsule membrane wall and, consequently, the smaller is the pore space between chitosan molecules, making it harder for citronella oil to be released from these microcapsules.

The effect of the size of these microcapsules on the oil release was studied when using $0.5 \mathrm{wt} \%$ chitosan, adding $1.0 \mathrm{wt} \% \mathrm{NaOH}$, and changing the agitation speed. From Figure 17, it was found that by increasing the emulsion stirring speed, microcapsules with smaller sizes are produced, and the release rate of citronella oil is increased.

In a recent study, $\mathrm{Mu}$ et al. developed a simple and flexible method for obtaining chitosan microcapsules with structures and functions that can be controlled via the interfacial cross-linking reaction of water-in-oil (w/o) emulsion models [45]. The size and thickness of microcapsule wall were studied, and it was verified that both are affected by the preparation condition. However, changes in size and wall thickness of chitosan microcapsules prepared under continuous conditions are much faster than those prepared in batches. The microcapsule release behaviour was also studied. It was concluded that this work provides valuable guidance for favourable control in the manufacture of chitosan microcapsules with structures and functions designed using emulsion models.

The controlled release behaviour of fluorescein isothiocyanate- (FITC-) dextran as a drug model for chitosan microcapsules with varying shell thickness is shown in Figure 18. A dynamic dialysis method was used to determine the drug release behaviour, in order to study the influence of 
TABLE 1: Size, surface charge potential $(\xi)$, and efficiency of encapsulation (EE) of the eugenol-loaded CA, CAB, CAP, and EC microspheres, adapted from [31].

\begin{tabular}{|c|c|c|c|c|c|c|}
\hline Sample & $d_{10}(\mu \mathrm{m})$ & $d_{50}(\mu \mathrm{m})$ & $d_{90}(\mu \mathrm{m})$ & Mode $(\mu \mathrm{m})$ & $\xi(\mathrm{mV})$ & $\mathrm{EE}(\%)$ \\
\hline $\mathrm{CA}$ & $0.54 \pm 0.02$ & $1.21 \pm 0.01$ & $4.87 \pm 0.02$ & 1.26 & $-28 \pm 1$ & $34.7 \pm 0.1$ \\
\hline $\mathrm{CAB}$ & $0.98 \pm 0.01$ & $2.85 \pm 0.01$ & $7.22 \pm 0.02$ & 2.19 & $-29 \pm 1$ & $24.6 \pm 0.2$ \\
\hline CAP & $8 \pm 1$ & $20 \pm 1$ & $30 \pm 4$ & 19.95 & $-30 \pm 1$ & $31.4 \pm 0.1$ \\
\hline EC & $3 \pm 1$ & $12 \pm 1$ & $29 \pm 1$ & 11.48 & $-32 \pm 1$ & $18.5 \pm 0.3$ \\
\hline
\end{tabular}

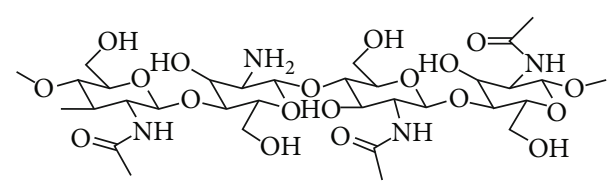

Figure 13: Chemical structure of chitin.

microcapsule structure on its release performance. The drug release is achieved by simple diffusion conducted in the concentration gradient, without rupture of the microcapsule structure.

The microcapsule release profiles exhibit a similar trend and fit a first-order kinetic model, being the cumulative release ratios of FITC-dextran from microcapsules with relative shell thickness of $18.8,24.7$, and $43.3 \%$, respectively, $91.0,89.9$, and $84.2 \%$ in 90 minutes. It is possible to determine the release rate through the difference in concentrations between the inside and outside of microcapsule. This causes microcapsules with different relative shell thicknesses to show different release rates. It can be seen in Figure 18(b) that cross-linked microcapsules showed a relatively smaller thickness. Consequently, this material had a greater capacity of drug loading and exhibited a faster initial release rate. In conclusion, these results demonstrate that drug loading capacity and its release behaviour can be controlled by tailoring the relative thickness of microcapsule shell.

(4) Alginate. Alginate (Figure 19) is a natural biopolymer synthesized from algae and microorganisms that are present in cell walls and in cell capsules of some bacteria. It forms water-soluble salts and low molecular weight amines. Alginate has been studied to form gels after the introduction of counterions. Its cross-linking degree depends on several factors, such as $\mathrm{pH}$, counterion, and functional charge density [46-51]. These gels have been used in controlled drug delivery systems and to encapsulate several herbicides, microorganisms, and cells [52-57].

The biocompatibility of alginate is a key factor for the administration of drugs. Alginates have contaminants that can induce toxic immunogenic responses in the body [58-62]. Through reactions catalysed by acids or alkalis, thermal depolymerization, or by polymer modification, alginate degradation occurs as a result of cleavage of glycosidic bonds. The mannuronic acid-rich (M-rich) alginates are more susceptible to acid and thermal degradation than the guluronic acid-rich (G-rich) alginates [63].Alginases are enzymes present in alginates, which makes them susceptible to biodegradation. These enzymes are present in seaweed, marine molluscs, and microorganisms and work at neutral $\mathrm{pH}$ [64]. Another characteristic of alginate is that it is one of the biomaterials responsible for the delivery of bioactive molecules, proteins, genes, and other drugs [65].

There are several strategies in administration of drugs that can be illustrated using polysaccharide-based systems. Specific transport of drugs to the desired location represents a huge field of research. The two main objectives of microencapsulation for drug administration are

(a) Safe transport of active molecule to the target site

(b) Minimal impact on the biological system of host

Alginate has become a more popular biomaterial in microencapsulation due to its high biocompatibility and low cytotoxicity [66]. Zhao et al. prepared mechanically resistant alginate microcapsules with the purpose to encapsulate and protect bacteria (Lactobacillus plantarum ST-III) from lactic acid (LA) [67]. By controlling the viscosity and gelation capacity of alginate solution, a series of microcapsules were prepared with same particle size and variable mechanical strength. These microcapsules were characterized by optical microscopy (Figure 20), showing that projected microcapsules have spherical shape, with negligible aggregation or agglomeration.

Diel et al. produced alginate microcapsules by electrostatic extrusion technique containing baby hamster kidney (BHK) recombinant cells that overexpress $\alpha$-L-iduronidase (IDUA) [68]. The optimization study was carried out through an experimental Box-Behnken project that allowed simultaneous assessment of influence of tension, alginate/ cell suspension flow, and alginate concentration in IDUA size and activity. This study presents an alternative for the treatment of mucopolysaccharidosis type I (MPS I), which is an autosomal recessive disease caused by IDUA deficiency, and results in lysosomal accumulation of glycosaminoglycans (GAG), which lead to generalized clinical manifestations (Figure 21).

Sun et al. developed microcapsules of sodium alginate and pectin as a matrix to encapsulate the antimicrobial agent carvacrol using a spray drying method [69]. Physical properties, microstructure, encapsulation efficiency, and stability of microcapsules were evaluated, as well as antimicrobial activity and antioxidant capacity of the products. SEM results revealed little evidence of roughness or cracking in the 


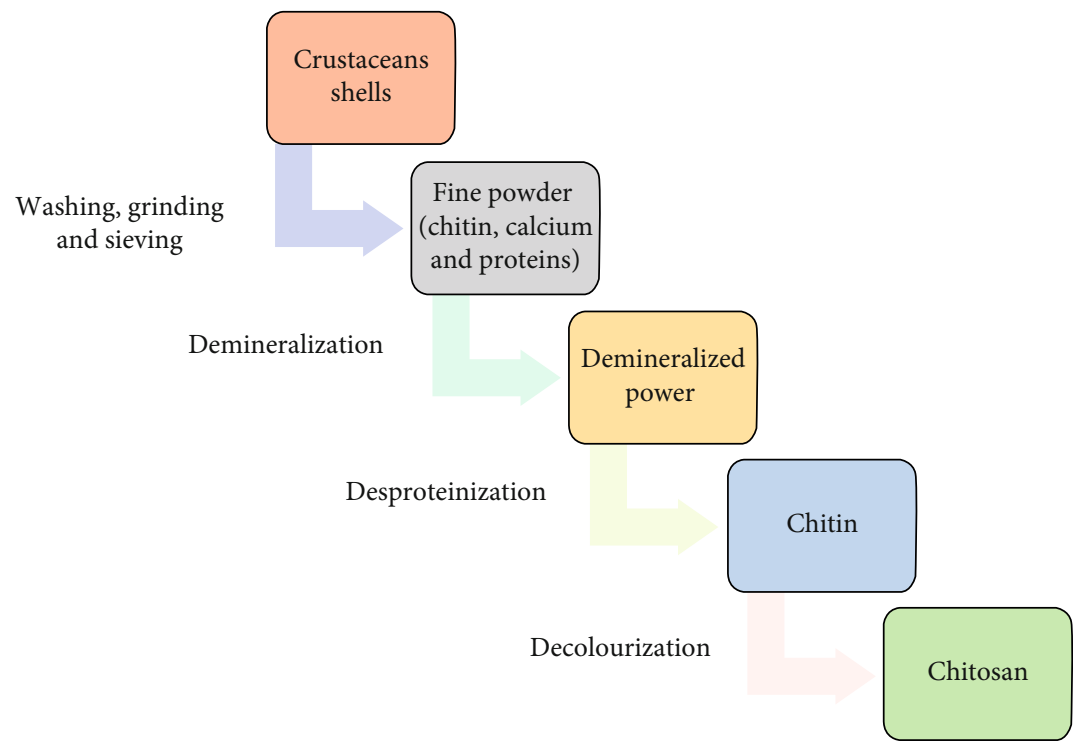

FIgURE 14: Extraction procedures for chitin and chitosan.

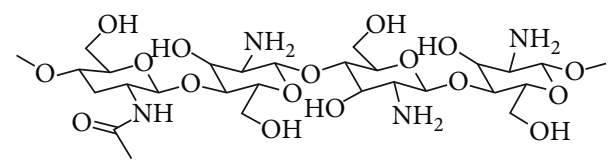

FIgURE 15: The chemical structure of chitosan.

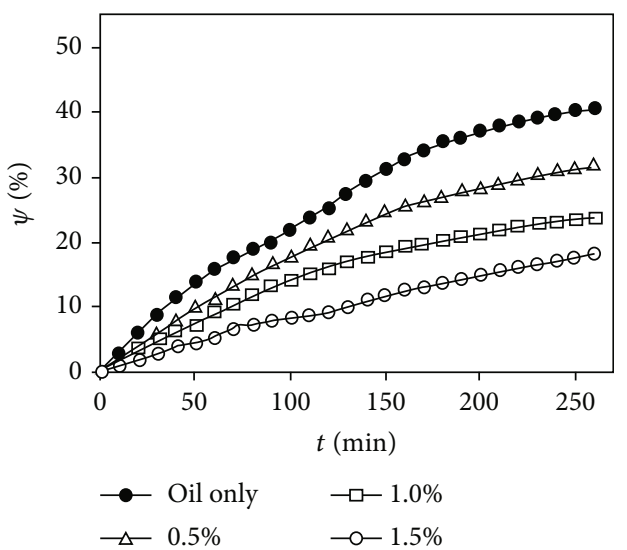

FIgURE 16: Time courses of oil release from microcapsules at different concentrations of chitosan. The symbols (black circle), (triangle), (square), and (white circle) denote concentrations of chitosan of $0 \mathrm{wt} \%$ (oil only), $0.5 \mathrm{wt} \%, 1.0 \mathrm{wt} \%$, and $1.5 \mathrm{wt} \%$, respectively [44].

microcapsules, which suggests a high stability of encapsulated carvacrol. It was also observed that the resulting microcapsules possess a well-defined spherical shape with regular particle sizes. The results of this study also indicated a high encapsulation efficiency (76.98\%) and stability. Furthermore, the results suggested that microencapsulation did not significantly affect antimicrobial activity against Escherichia coli and antioxidant activity of carvacrol. This work allowed to achieve a stable release of carvacrol suggesting

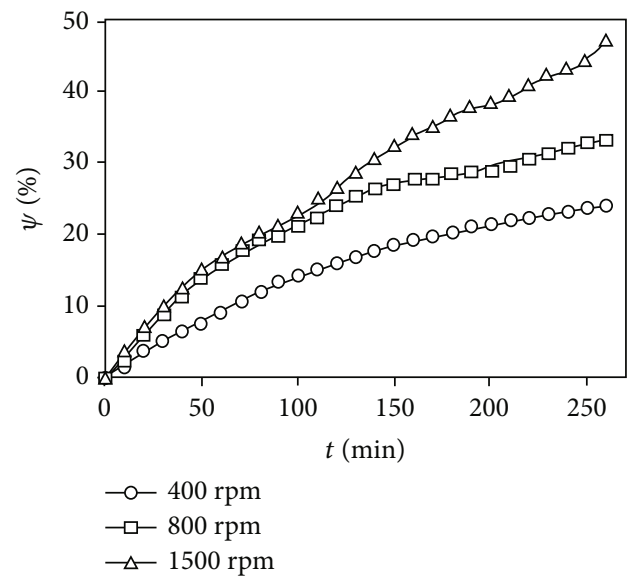

Figure 17: Time courses for citronella oil (0.5 wt\%) release from chitosan microcapsules prepared at varying emulsion stirring rates [44].

that it may be potentially used as a controlled release decontaminator in food processing and nutraceutical industries (Figure 22).

The authors of this review highlight the feasibility of applying this microencapsulation system to the carvacrol encapsulation. The research presents several study variables that successfully prove the encapsulation of this phenolic compound, such as the efficiency of microencapsulation, stability, microstructure, and the study of maintenance of antimicrobial and antioxidant properties of carvacrol. It should be noted that this matrix of microcapsules with peptin/ sodium alginate could be used to encapsulate other substances, considering its applicability and compatibility when used in processed foods, dairy products, or nutraceutical items. Microcapsules formed by pectin and alginate are therefore a promising option for application in the food and pharmaceutical sector. The pectin-alginate matrix is 


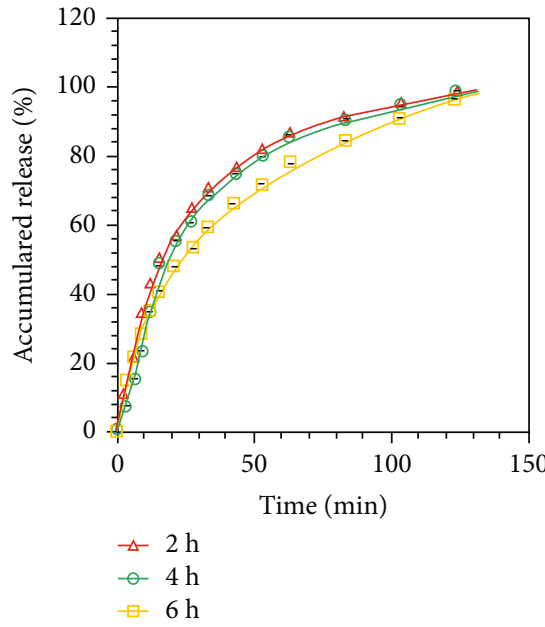

(a)

FIGURE 18: Cumulative release curves (a) and release rate curve thicknesses at room temperature [45].

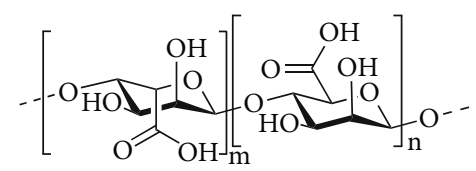

Figure 19: Chemical structure of alginic acid.

relatively inexpensive and, mainly, nontoxic and biocompatible. Several essential oils with antimicrobial and antioxidant activity important for food and pharmaceutical products are not used due to their high volatility and low solubility in water, which can be overcome by using this matrix in the microencapsulation system.

2.1.2. Protein-Based Polymers. Proteins are generally used in the natural form in the environment, since in most cases they are not soluble or bonded without degradation. Proteins are copolymers with arrangements of different types of amino acids, making their biosynthesis a complex process that involves different types of enzymes. The enzymatic degradation of protein, using proteases, is an amide hydrolysis reaction.

(1) Gelatin. Gelatin (Figure 23) is an animal protein that consists of 19 amino acids linked by peptide bonds, being hydrolysed by several proteolytic enzymes to produce amino acids [70]. Considering that it is a biodegradable and watersoluble polymer, gelatin is used in distinct industrial fields, like coatings, microencapsulation $[16,71-76]$, and in preparation of biodegradable hydrogels [77-80].

Gelatin can be produced from the skins of cold and hot water fish species, birds, hides, skins, and bones from mammals (pigs and cattle). What these products have in common is that they all contain collagen. Gelatin manufacturing process consists of cleaning the tissues, followed by pretreatments, gelatin extraction, filtration/purification/sterilization, drying and, finally, grinding. The collagen monomer (tropocollagen) is a triple helical stem composed of three parallel $\alpha$-chains.

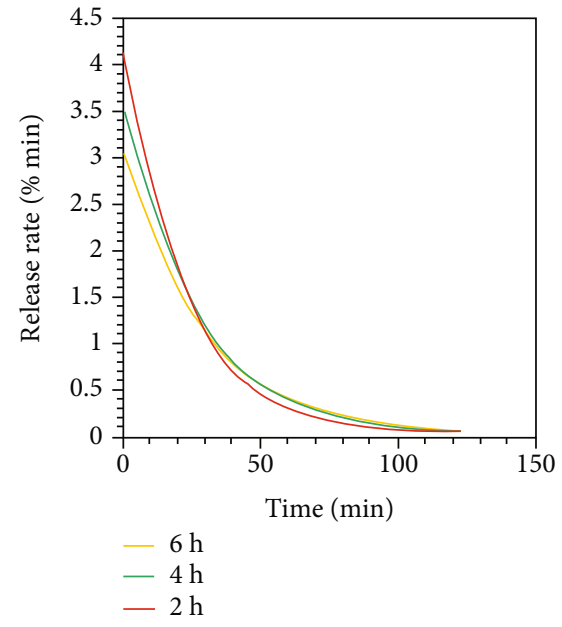

(b)

(b) of FITC-dextran-loaded chitosan microcapsules with different This protein is insoluble in water, but in small fractions, it is soluble in dilute acid or base. With the aging processes that occur in mammals, a decrease in collagen solubility will occur, which is due to an increasing number of covalent bonds that are formed in tissue after aging.

From several scientific studies, scientists have shown that thermal stability of collagen is related to content of pyrrolidine [81-85], and this content is one of main factors that determine the physical properties of gelatin. Some important properties related to gelatin gelation process are gel formation, texture, thickening, and binding to water.

Gelatin has been used to encapsulate different food ingredients through three technologies (spray drying, gelation, and coacervation) due to its chemical and physical properties.

(a) Spray drying

For the encapsulation of food ingredients, spray drying is the most common method. Gelatin microcapsules are good carriers of food ingredients due to their emulsifying properties. Regarding lipophilic food ingredients, these are usually emulsified before being encapsulated. This is the most suitable for microencapsulation in the spray drying process due to its film forming properties $[86,87]$.

In spray drying microencapsulation (Figure 24), gelatin is used with hydrocarbon compounds [88], and sucrose is used as a plasticizer, which was believed to promote the formation of spherical and smooth surface microcapsules [89].

\section{(b) Gelation}

The gelation properties of gelatin make this polymer the most used in microencapsulation. Gelatin gels show high permeability and dissolve in hot conditions, making different approaches for food ingredients encapsulation. There are two other ways to use gelatin gel. One that incorporates main materials into the gelatin solution and induces gelation and gel cross-link [91-94] or, alternatively, to prepare 


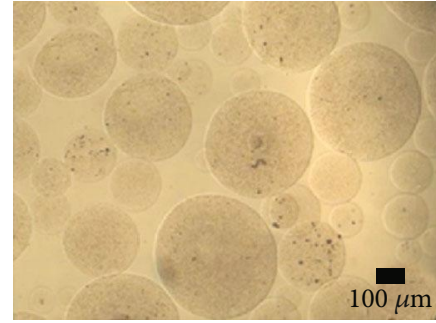

(a)

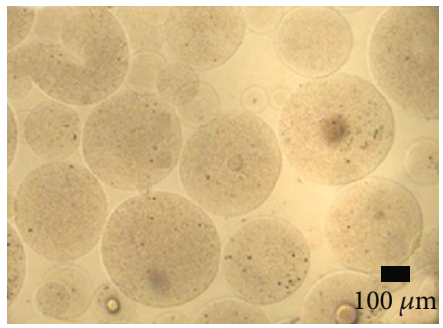

(c)

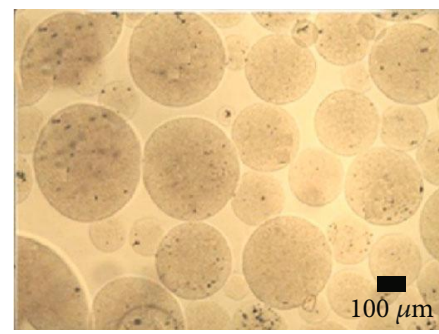

(e)

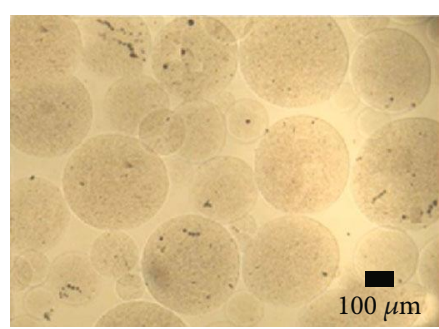

(g)

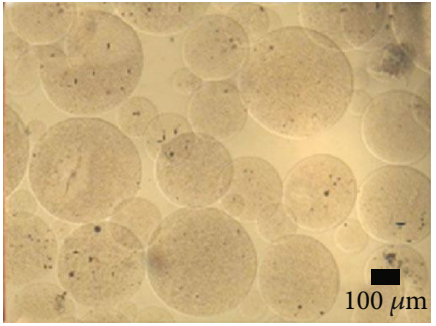

(b)

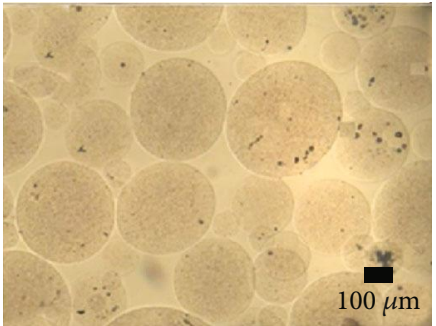

(d)

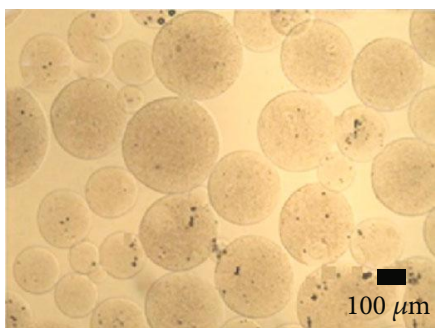

(f)

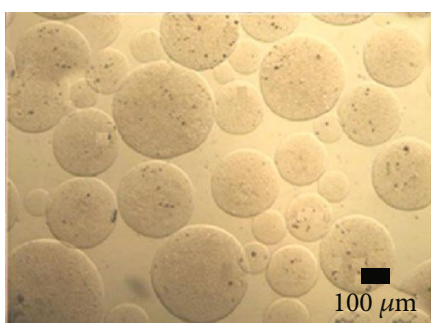

(h)

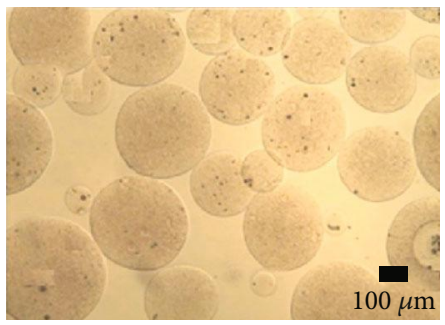

(i)

Figure 20: Optical micrographs of alginate microcapsules produced with different procedures, diameter, span factor, and mechanical strength [67].

gelatin microspheres and then load them with food ingredients. In the preparation of gelatin microspheres, it is necessary to use gelatin-induced phase separation $[95,96]$. Iwanaga et al. prepared gelatin microspheres using the water-in-water $(\mathrm{w} / \mathrm{w})$ emulsion technique, and the results showed that gelatin microspheres could be loaded with ingredients by diffusion [95, 97-99].

\section{(c) Coacervation}

With an insoluble layer of protein around fine droplets of core material, the formation of microcapsules by proteins may be possible through the simple or complex coacervation technique. In simple coacervation, compounds with high affinity for water are added; while in complex coacervation, 


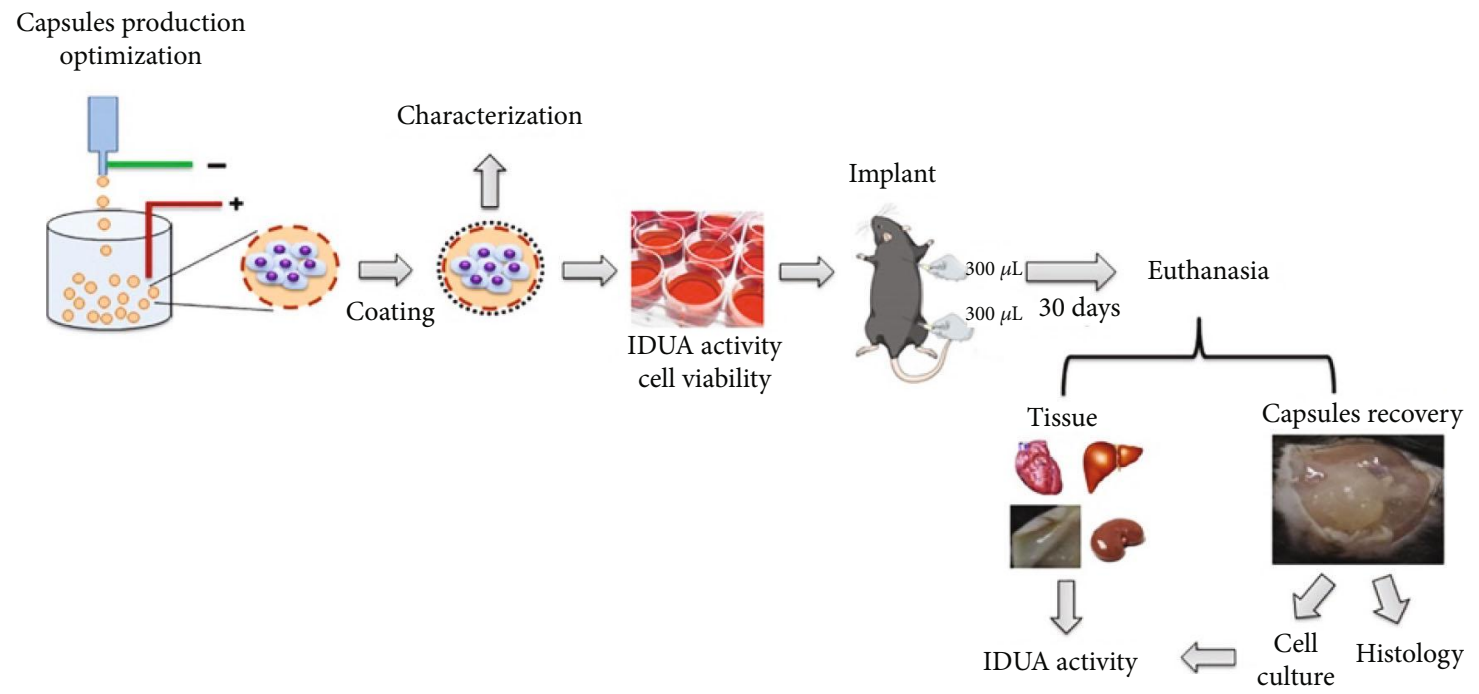

FIGURE 21: Schematic illustration of the fabrication of alginate microcapsules and in vivo and in vitro characterization [68].

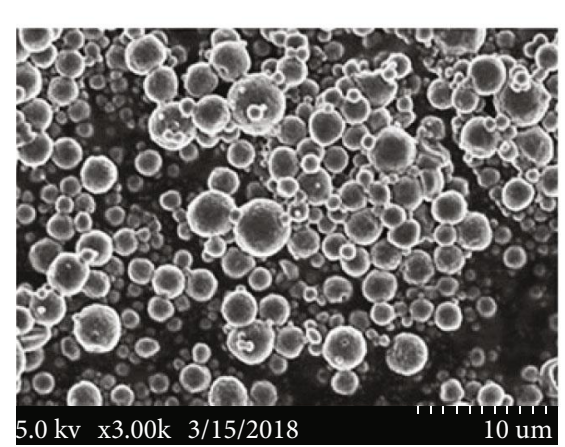

Spray-dried carvacrol in pectin-alginate microcapsules
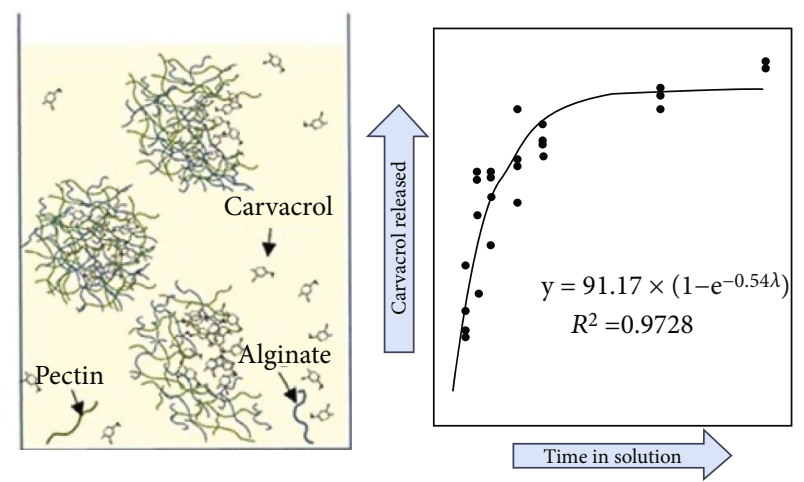

Release of carvacrol from pectin-alginate matrix into solution

FIGURE 22: (left) SEM micrograph of carvacrol in pectin-alginate matrix microcapsules (3000× magnification) and (right) release of carvacrol from pectin-alginate microcapsules in potassium phosphate buffer solution ( $\mathrm{PBS}, \mathrm{pH}=7.4$ ) at $37^{\circ} \mathrm{C}$ [69].

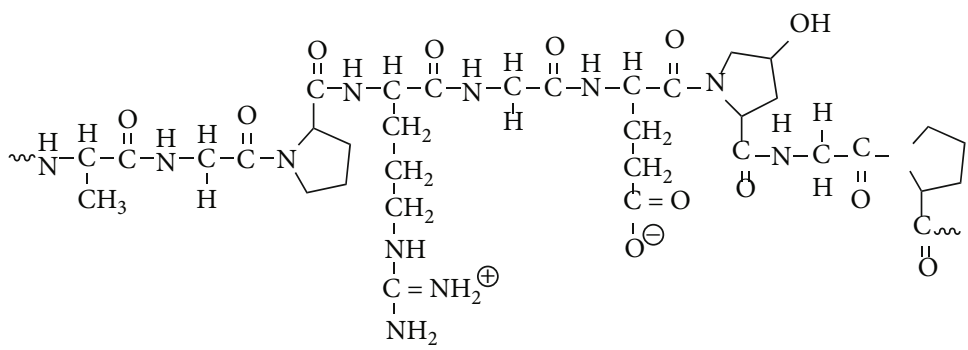

Figure 23: Representative gelatin structure; Ala-Gly-Pro-Arg-Gly-Glu-4Hyp-Gly-Pro.

a protein-rich layer is formed around the core material by interaction with polymers of opposite charges [100]. As gelatin has been used in complex coacervation with different polyanions, several groups of researchers have encapsulated various food ingredients. Microencapsulation of vitamin A palmitate by gelatin-acacia [101] and microencapsulation of lycopene by gelatin-pectin [102] are examples of this technique.
These coacervation processes (Figure 25) can be controlled by $\mathrm{pH}$, polymer concentration, and stirring rate [103]. Nakagawa and Nagao developed a study using the freezing process to control the coacervation of gelatin complex [104]. Another aspect to be considered in the coacervation process is the use of surfactants, being used manly sodium dodecyl sulphate (SDS) $[100,105,106]$. 


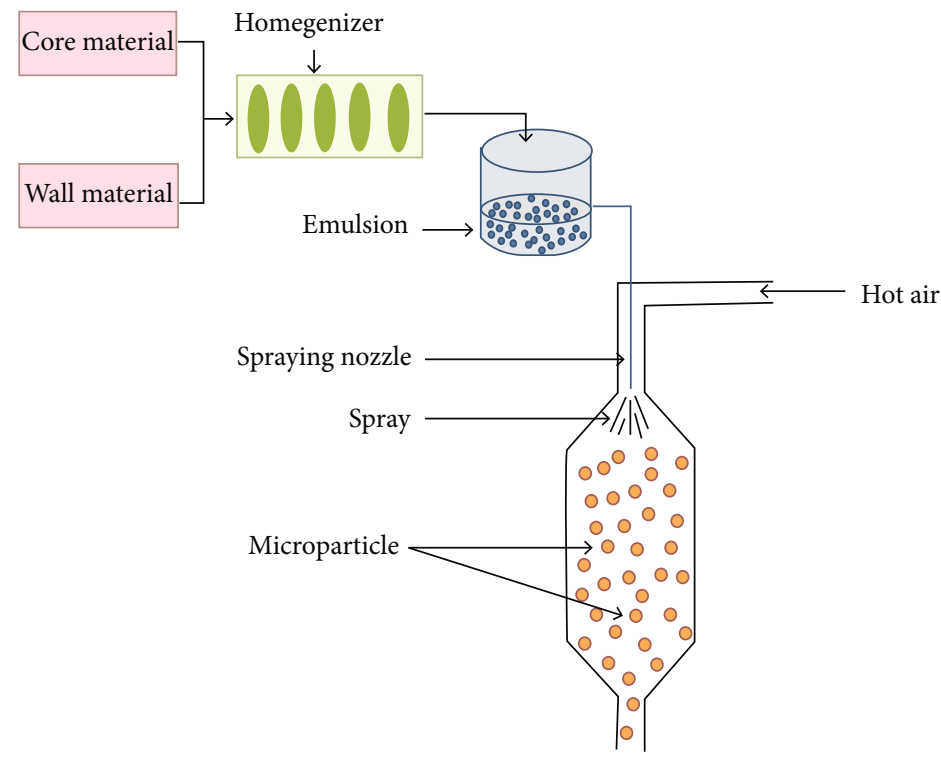

FIGURE 24: Schematic representation of the microencapsulation process by spray drying [90].

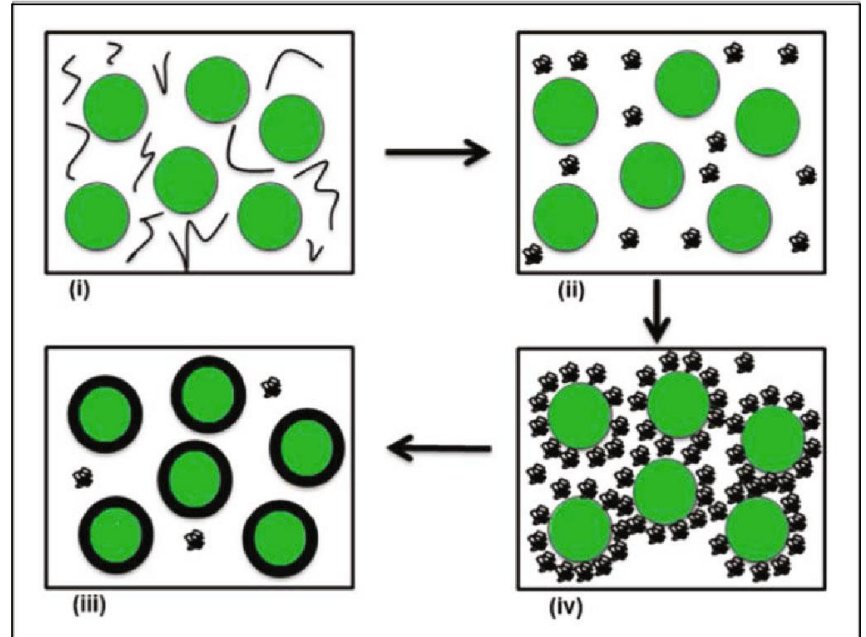

FIGURE 25: Example of complex coacervation involving (i) emulsification of oil in an aqueous solution containing two different polymers, (ii) initial coacervation of polymers after lowering the solution $\mathrm{pH}$ below the isoelectric point of protein, (iii) deposition of the polymers on the surface of the oil, and (iv) wall hardening by addition of a cross-linking agent. [90].

To improve the retention of drugs in nasal cavity and the resulting pharmacokinetic profile, the use of mucoadhesive microparticles has become essential. Lim et al. studied and compared several types of microspheres with potentially mucoadhesive properties, prepared by solvent evaporation (Figure 26), composed of hyaluronic acid (HA), chitosan glutamate $(\mathrm{CH})$, and a combination of the two with HA microcapsules and gelatin prepared by complex coacervation (Figure 27) [107]. In vitro release profiles of microsphere formulations prepared by solvent evaporation were determined.

In Figure 28, it is shown the percentage of gentamicin released from microsphere formulations determined by fluorescence polarisation immunoassay (FPIA). The release of gentamicin sulphate was not complete from microspheres within 5 hours, but most of microspheres had dissolved within 1 hour, and some remnants were still appeared after 5 hours. During solvent evaporation process, some denaturation may have occurred as the polymers used are all soluble in their native state. For this reason, it is possible that gentamicin sulphate with a positive charge is associated with the traces of microspheres, limiting its release in solution. This work showed that $\mathrm{HA}$ and $\mathrm{HA} / \mathrm{CH}$ have a longer release, causing the release rate to be divided into an initial phase and a terminal phase.

In order to improve the appeal of frozen roasted foods during heating, Yeo et al. encapsulated flavour oil inside of gelatin and arabic gum microcapsules using complex coacervation method [108]. The release of oil from the formulations was determined by a simple spectroscopic method, based on the separation of oil marked with a lipophilic dye from unaffected particles (Figure 29). Almost of all 


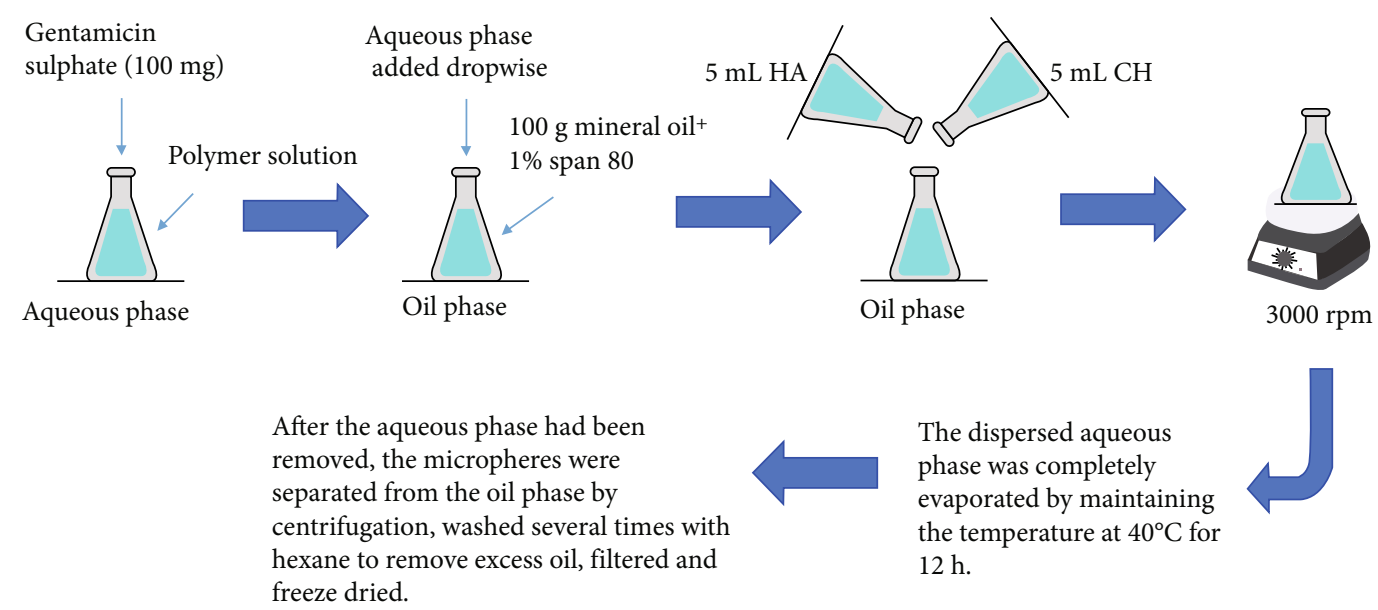

FIGURE 26: Schematic representation of water-in-oil (w/o) emulsification solvent evaporation technique [107].

$\begin{array}{ll}\begin{array}{l}\text { A specific ratio of each } \\ \text { polymer solution giving a total } \\ \text { polymer solution volume of } 200 \\ \text { ml was mixed in a } 500 \mathrm{ml} \text { beaker }\end{array} & \begin{array}{l}\text { 1. The } \mathrm{pH} \text { of the solution was } \\ \text { theduced using } 0.1 \mathrm{M} \mathrm{HCl}\end{array} \\ \text { to accurred }(\mathrm{pH} \mathrm{3.6})\end{array}$

The primary coacervating polymer solutions were prepared by hydrating separately in distilled water for $12 \mathrm{~h}$ at $40^{\circ} \mathrm{C}$

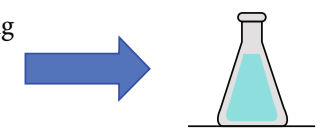

Mechanical stirrer at $300 \mathrm{rmp}$ for $5 \mathrm{~min}$
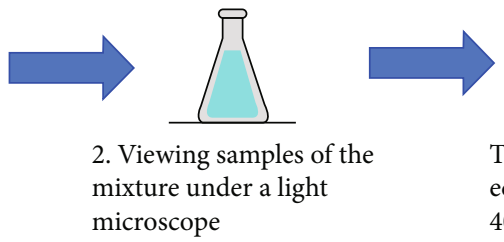

The coacervate was allowed to equilibrate for $30 \mathrm{~min}$ and at $40^{\circ} \mathrm{C}$
1. The coacervate suspension was then centrifuged at $1000 \mathrm{rpm}$ for $10 \mathrm{~min}$.

2. The microcapsules were subjected to washings with cold distilled water, centrifuged at $1000 \mathrm{rmp}$ fore $5 \mathrm{~min}$ and the supernatant discarded.

3. The washing procedure was repeated with a series of cold isopropyl alcohol solutions, at concentratoions of 50,75 and $100 \%$ in water, after which recovered microcapsules were freezed dried.
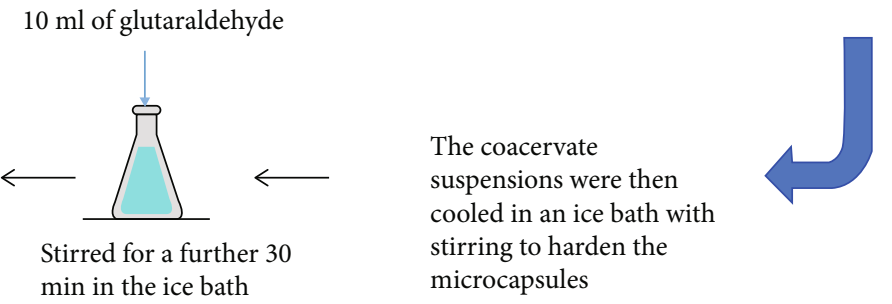
stirring to harden the microcapsules

FIGURE 27: Schematic representation of the HA/gelatin and acacia/gelatin microcapsules using a complex coacervation method [107].

encapsulated oil was released when microcapsules were heated to $100^{\circ} \mathrm{C}$ or above. The remaining encapsulated oil was maintained in storage for 4 weeks at $4^{\circ} \mathrm{C}$ and $-20^{\circ} \mathrm{C}$, but was released upon exposure to $100 \mathrm{mM} \mathrm{NaCl}$ at room temperature. When these particles were cooled after the release of their oil content, the oil was re-encapsulated.

To find out if the previous microencapsulation system could be recycled, this work by Yeo et al. included the study of particles that were heated and then cooled in an ice bath. Through optical microscopy, it was observed that gelatin and arabic gum microcapsule walls that were previously destroyed by heating were reformed after cooling (Figure 30). Due to the increase in the number of encapsulated oil droplets, there was a marked increase in the size of capsule (Figure 30(a) versus Figure 30(b)). In Figure 30(b) is shown that the particles were formed when well-suspended particles were heated and then cooled. The same phenomena happened when particles were aggregated by sedimentation before heating (Figures 30(c) and $30(\mathrm{~d}))$. This condition can be a more accurate reflection of what can occur in a food product.

Analysing this study, the materials used for microcapsules, arabic gum, and gelatin are indicated for food application due to their chemical nature (nontoxic and nonirritable), their availability in food grade, and the fact that solubility is dependent on temperature, due to gelatin. In addition, developed microcapsules do not require exhaustive purification processes. However, some of the developed formulations show a significant aggregation of microcapsules. Despite not having a considerable impact for the mentioned application, these formulations can be improved 


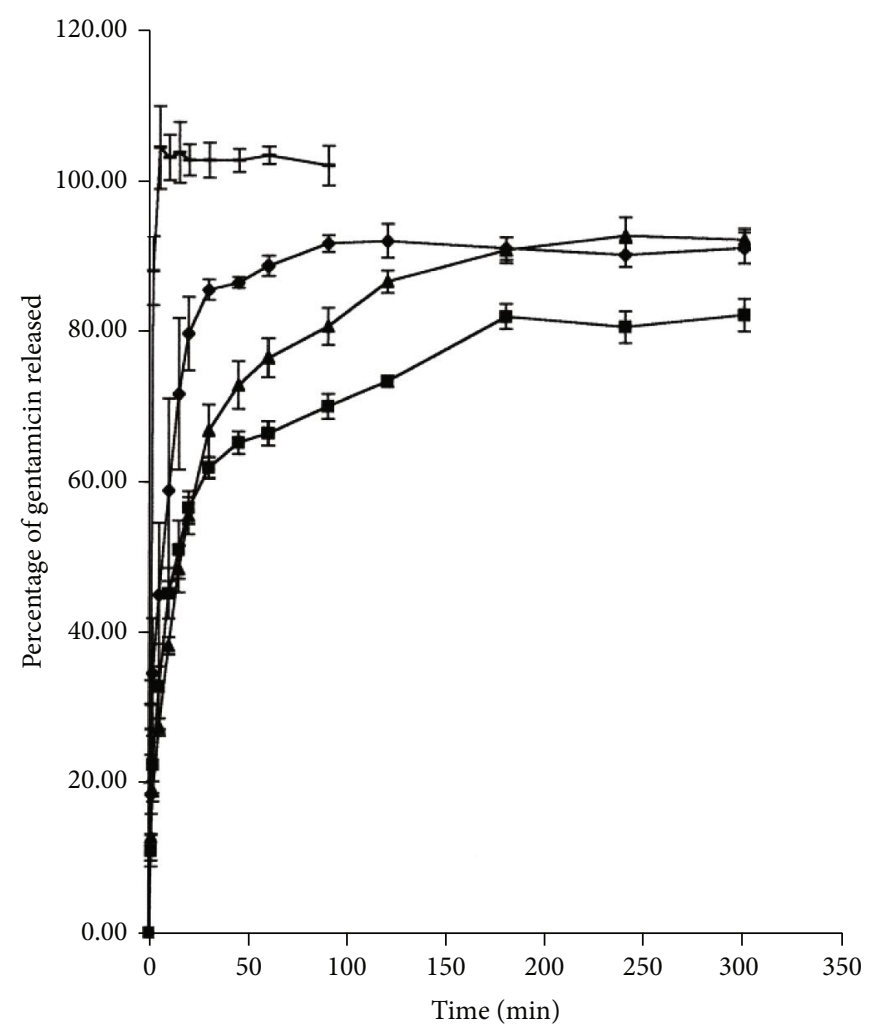

FIGURE 28: Release profiles of gentamicin sulphate from microsphere formulations: (『) $\mathrm{CH}$; $(\mathbf{\square}) \mathrm{HA} / \mathrm{CH}$; $(\mathbf{\Delta}) \mathrm{HA}$; (一) gentamicin/lactose; mean \pm S.D. $(n=6)[107]$.

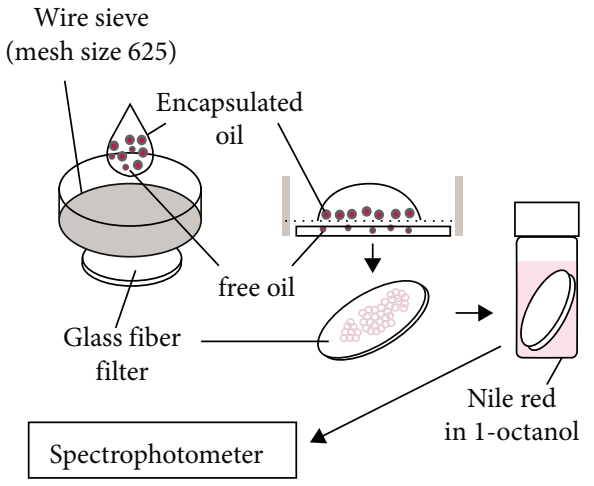

Figure 29: Schematic of the measuring of released bake flavour oil [108].

through the optimization of experimental parameters during production, allowing its use in other applications. Furthermore, it would be interesting to test the use of other acids (such as citric acid) to avoid adding flavour effects. It would also be important to change the method for determining the encapsulation efficiency and release behaviour of encapsulated oils. This change would allow to distinguish between release due to particle rupture and release by diffusion through the hydrophilic particle shell in components with high water solubility.

Liu et al. obtained microcapsules of chitosan-gelatin soaked in patchouli oil that were prepared by complex method of copreservation (Figure 31) [109]. The surface and morphology of microcapsules were characterized by SEM, and it was verified that the microcapsules have a regular spherical shape and diameters in the range of 1 to $20 \mu \mathrm{m}$. Regarding thermal stability, microcapsules remained stable below $190^{\circ} \mathrm{C}$, which indicates that fabric can be finished at $160^{\circ} \mathrm{C}$. Finally, encapsulation efficiency and load capacity of microcapsules were calculated at $50.69 \%$ and $30.31 \%$, respectively.

Figure 32 shows the results of surface morphology of microcapsules using different emulsification methods. Figures 32(a) and 32(b) have different emulsification methods but both show similar morphology, spherical and rough, due to dehydration during the freeze-drying process. However, the microcapsules of Figure 32(b) show better uniformity and dispersion and no aggregation. This can be related with the more uniformity and homogeneous shear emulsification forces. Figure 32(c) shows some dimples and fragments when compared with Figure 32(d). Finally, comparing the particle size of microcapsules by SEM and by dynamic laser scattering (DLS), the latter was slightly larger, showing that the microcapsule wall materials are hydrophilic polymers and have a strong swelling in water.

Fraj et al. produced microcapsules for simultaneous encapsulation of hydrophilic and lipophilic active substances [110]. As hydrophilic and lipophilic compounds, vitamins C and $\mathrm{E}$ were used, using the double emulsification process, followed by complex coacervation of two polymers, gelatin and sodium caseinate (NaCAS). According to the characterization of microcapsules, such as surface morphology 


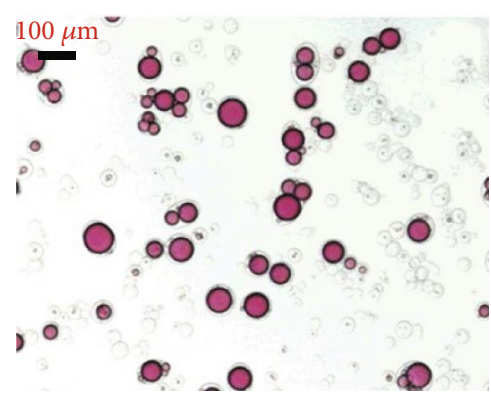

(a)

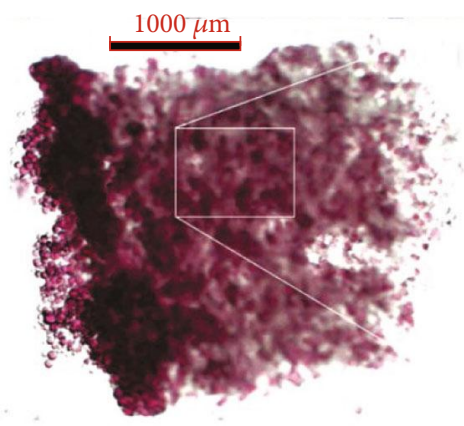

(c)

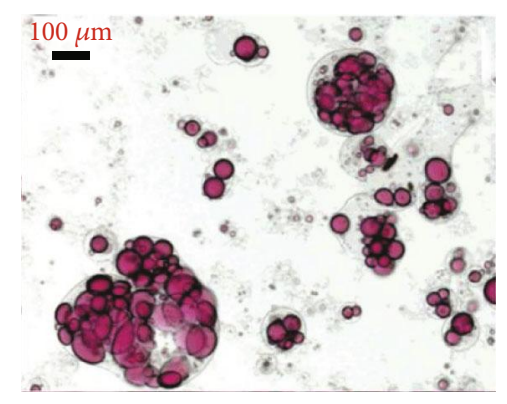

(b)

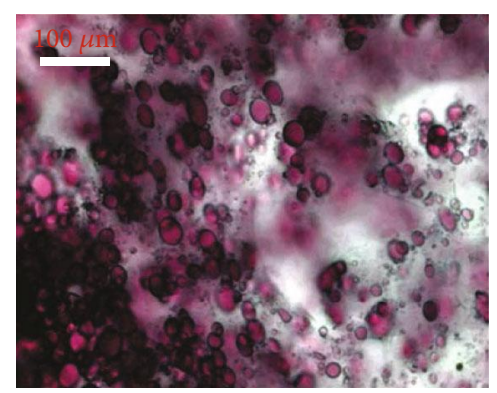

(d)

Figure 30: Recoacervation of heated microcapsules upon cooling [108].

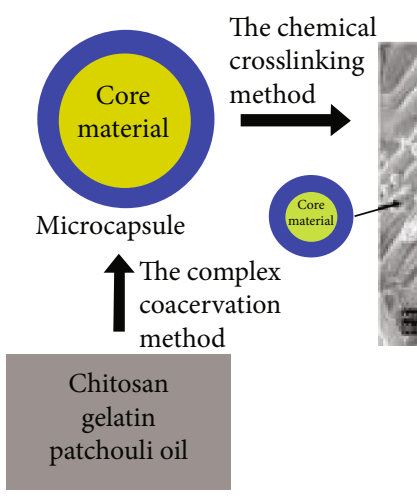

Figure 31: Schematic representation of the chitosan-gelatin microcapsules and crosslinking method [109].

(Figure 33), encapsulation efficiency and vitamin $\mathrm{C}$ release kinetics, showed that through the concentration of genipin and interaction in the gelatin/NaCAS system, the properties of microcapsules were influenced.

According to the encapsulation efficiency (EE) results, these showed that EE was significantly higher for vitamin C compared to vitamin E (Figure 34). This system for microencapsulation of lipophilic thermolabile compounds requires adjustment of conditions in the production, such as the use of freeze-drying processes instead of spray drying.

Araújo et al. developed microspheres of sweet orange essential oil using gelatin and maltodextrin as a polymeric wall, through emulsification/lyophilization [111]. In this study, the characterization of microspheres was performed in terms of yield, efficiency of microencapsulation, ultrastructural aspects, thermal analysis, infrared spectroscopy, and water activity. In order to assess bioactivity, the total phenolic content and antioxidant and antibacterial activity were studied.

The results demonstrated a high yield and efficiency of encapsulation, improvement of thermal stability, and maintenance of antibacterial and antioxidant properties of sweet orange essential oil. The presence of gelatin in the walls of the microcapsules positively influenced the properties of active agent, mainly its thermo-oxidative capacity. SEM micrographs (Figure 35) reveal that the maltodextrin walls have a smooth, pore-free, and sealed structure, and the gelatin walls have superficial pores that can be attributed to their hygroscopic characteristics. All samples have irregular microspheres, which can be attributed to the use of freeze drying as a drying method.

The microencapsulation systems developed in this study promoted a high stability of sweet orange essential oil, which is advantageous to protect against environmental factors and prolonged storage time.

\subsection{Synthetic Polymers}

2.2.1. Hydrolysable Polymers. As previously mentioned, there are natural polymers that can be harvested directly from nature. But, at the same time, there are polymers that are not available in nature, albeit can be produced with human intervention from natural sources.

A variety of biodegradable and biocompatible polymers are synthesized by chemical methods. Esters, anhydrides, and amides are the most common compounds, and the weak hydrolysable bonds that form the backbone of synthetic biopolymers is the main cause of biodegradability [112]. Decomposition can occur chemically or enzymatically in 


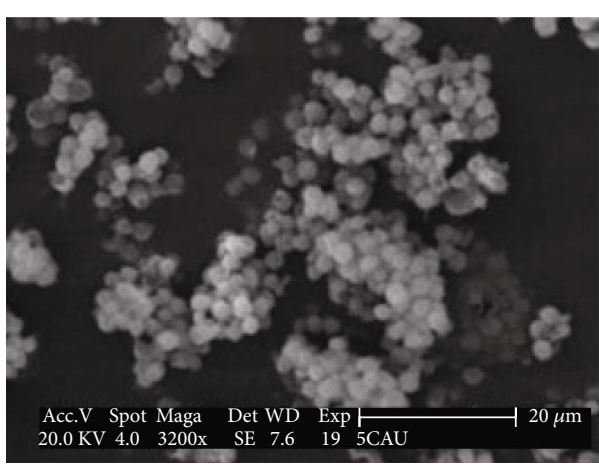

(a) $3200 \times$ magnification

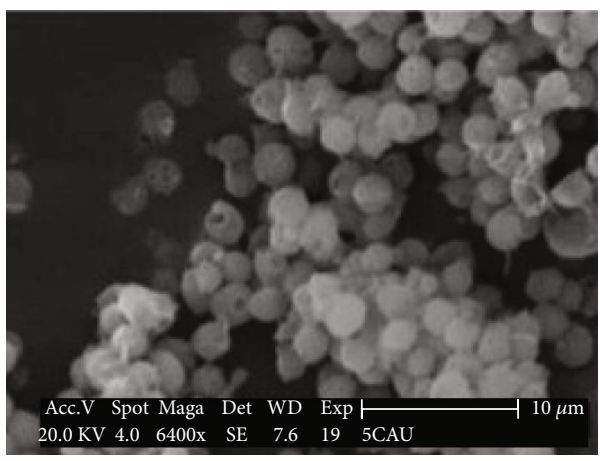

(c) $6400 \times$ magnification

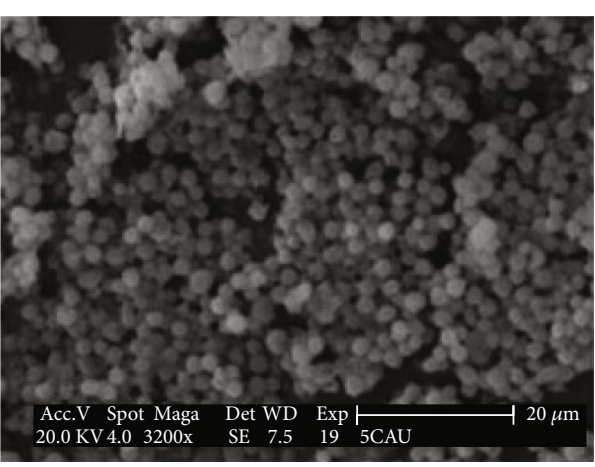

(b) $3200 \times$ magnification

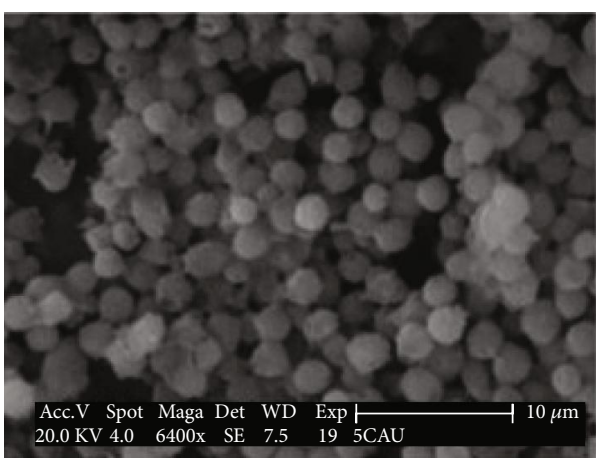

(d) $6400 \times$ magnification

FIGURE 32: SEM micrographs of chitosan-gelatin microcapsules produced by mechanical agitation emulsification (a, c) and shearing emulsification (b, d) [109].

its monomer units. Therefore, hydrolysable polymers can be biologically acceptable by the human body, which is essential for biomedical applications. Figure 36 shows the structures for some hydrolysable polymers.

(1) Polyesters. Polyesters are naturally occurring polymers that contain the functional ester group in their main chain. These polymers are biodegradable and there are also some synthetic polymers that can also be. However, most of synthetic polymers are not biodegradable.

The biodegradability of aliphatic polyesters is ascribed to an extremely hydrolysable backbone. For synthetic polyesters to be biodegradable, their polymeric chain must fit into the active enzyme site. This is one of the reasons why flexible aliphatic polyesters are degradable and rigid aromatic polyesters are not $[113,114]$. The two most used polyesters are poly (glycolic acid) and poly (lactic acid). Regarding its application, the polyesters are normally used in clothing.

$\mathrm{Yu}$ and $\mathrm{Zhu}$ introduced a new and easy route to manufacture polyester microcapsules [115]. The droplets of chloroform emulsion in water containing polyesters (poly(methyl methacrylate) (PMMA), poly(lactic acid) (PLA), or poly(lactic-co-glycolic acid) (PLGA)) selfemulsify in double emulsions simply by adding sodium dioctyl sulfosuccinate (Aerosol OT) to the chloroform. Microcapsules with controlled sizes and morphologies were obtained, and the wall thickness of the capsules was adjusted by varying the initial concentration of Aerosol OT or polymer.
Poly-DL-lactide (PDLLA) microcapsules are produced by means of solvent evaporation from emulsion droplets containing PDLLA and Aerosol OT. According to fluorescence and SEM micrographs, it was visualized that the wall thickness of the microcapsules is uniform and that the vesicle nature of the microcapsules can be resolved using fluorescence microscopy (Figure 37(a)) and SEM micrographs (Figure 37(b)). With the variation of the initial concentration of Aerosol OT or polymer, the wall thickness can be adjusted and other experimental factors (molecular weight of the polymer, temperature, and evaporation rate of the solvent) can play important roles in the structure and morphology of microcapsules.

The active agent (Congo Red) is encapsulated during the formation process and can be released gradually. Through the release profile from PMMA microcapsules, it can be seen that it has a release period of 40 hours (Figure 38 ). With this method, the authors suggested that microcapsules can be produced with different polymers for drug delivery and release applications.

Injectable and biodegradable microspheres of poly(lacticco-glycolic acid) (PLGA), which encapsulated a peptide model based on the luteinizing hormone releasing hormone (LHRHa) agonist leuprolide, were developed using the spray drying technique [116]. Several spray drying parameters were evaluated in order to prepare 1 month-controlled release formulations with a composition similar to the commercial Lupron Depot ${ }^{\circledR}$ (LD). The microspheres were characterized, and the long-term release was tested for 9 weeks in PBS + $0.02 \mathrm{v} \%$ Tween $80+0.02 \%$ sodium azide $\mathrm{pH} 7.4$ (PBST) at 


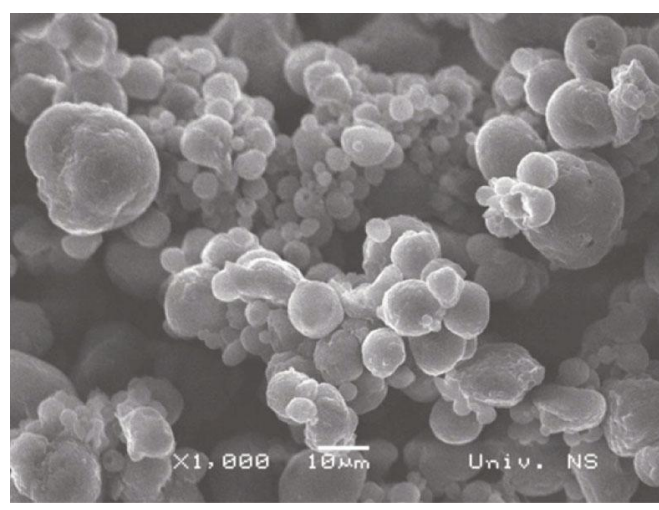

(a)

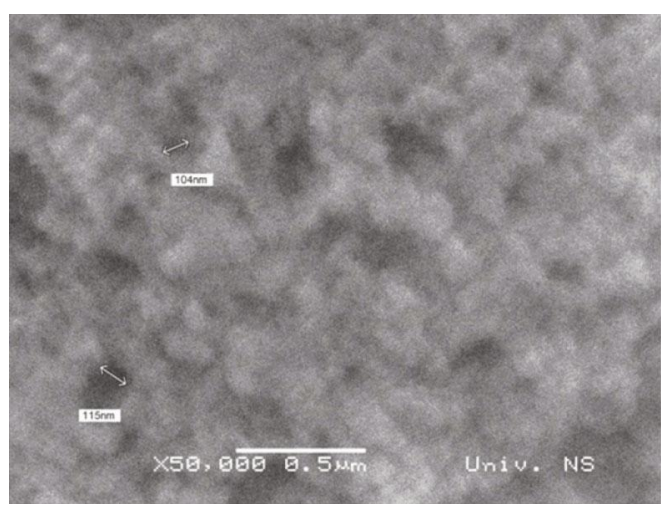

(c)

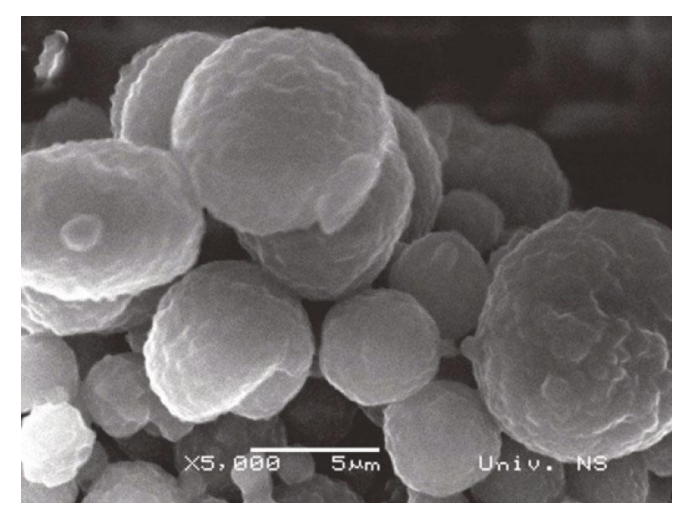

(b)

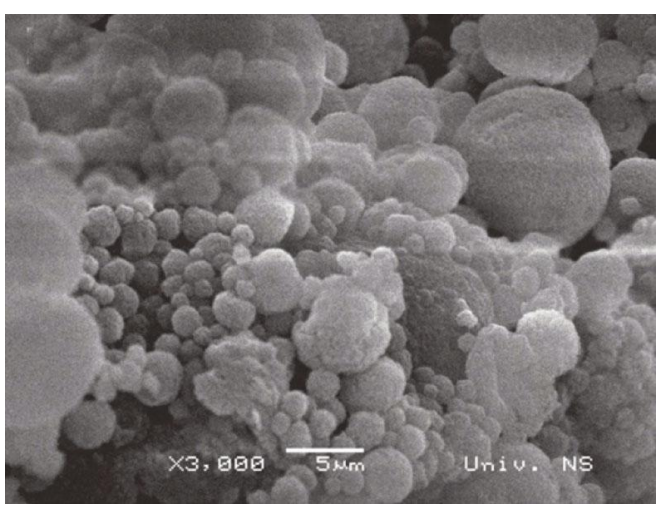

(d)

FIGURE 33: SEM micrographs of microcapsules obtained by cross-linking of gelatin: NaCAS coacervate for a mass ratio of $2: 1$, with $0.2 \mathrm{mmol}$ of genipin/g of proteins. Magnification of $1,000 \times$ (a); 5,000× (b); 50,000× (c). Microcapsules after active compounds release $(3,000 \times)(d)[110]$.

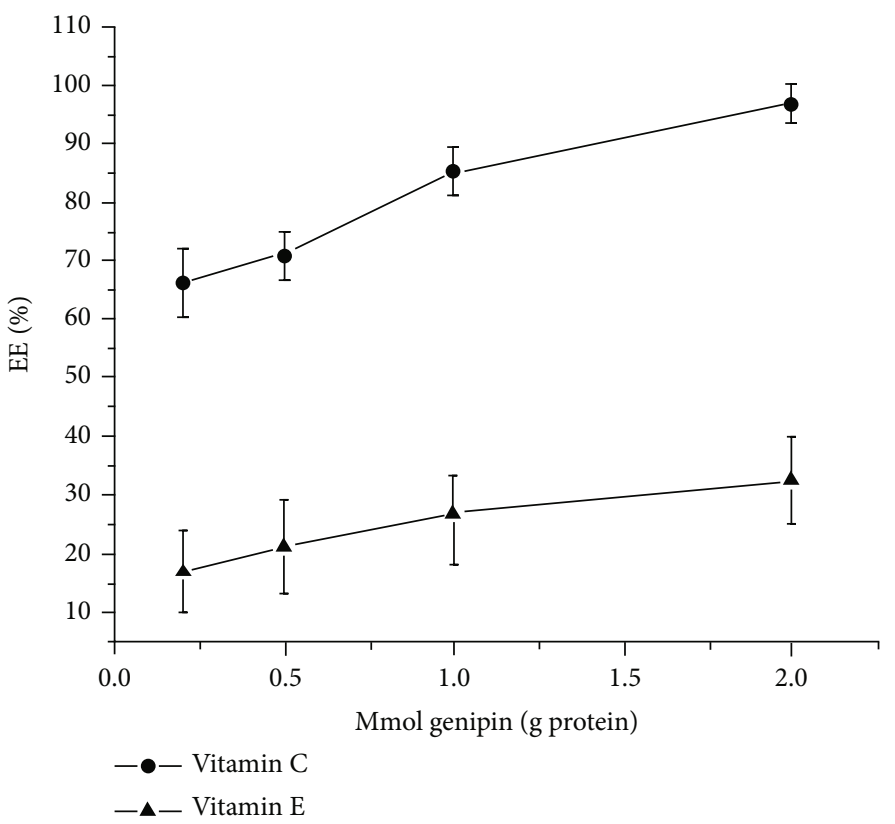

FIGURE 34: Effect of genipin concentration on the encapsulation efficiency (EE) of vitamin C and vitamin E in microcapsules [110].

$37^{\circ} \mathrm{C}$ (Figure 39). Comparing the microspheres obtained with the commercial LD microspheres, they presented highly similar or even improved long-term release profiles, providing via- ble alternatives for long-acting release (LAR) for LD. These data indicate that spray drying can be optimized to prepare PLGA microspheres formulations that are commercially 


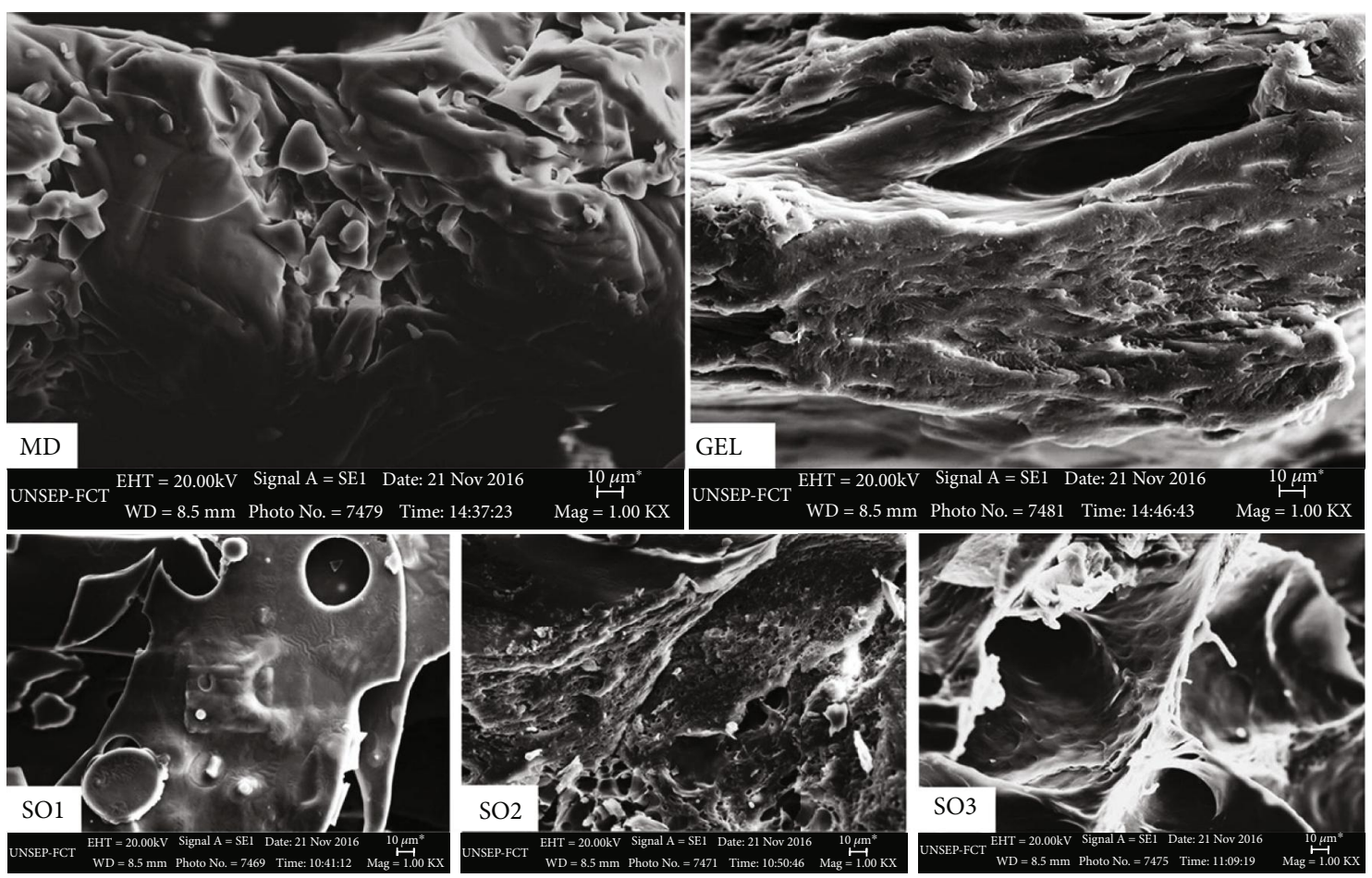

FIGURE 35: SEM micrographs of the isolated wall materials, maltodextrin (MD) and gelatin (GEL), and sweet orange essential oil microencapsulated systems (SO1, SO2, and SO3). SO1: contains only MD; SO2: contains $\mathrm{MD}$ and GEL at a ratio of 2:1; and SO3: contains MD and GEL at a ratio of $1: 1[111]$.

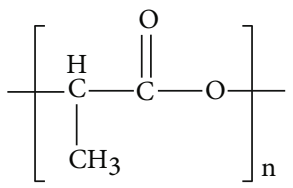

Poly (lactic acid)

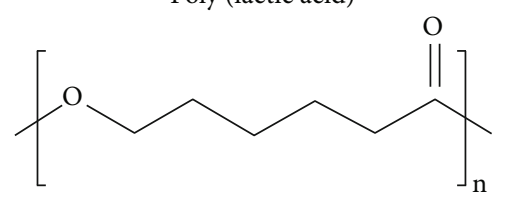

Polycaprolactone

FIgURE 36: Structures of poly(lactic acid) and polycaprolactone.

relevant for the delivery of peptides, including LHRHa and leuprolide.

\section{(a) Poly(glycolic acid) (PGA)}

Poly(glycolic acid) (Figure 40) is the simplest linear aliphatic polyester and can be prepared from glycolic acid through polycondensation or ring-opening polymerization. Its degree of crystallinity is high, around $45-55 \%$, which results in water insolubility [117].

As it is degradable, PGA is used as an absorbable thread and is being evaluated in the biomedical area. However, this rapid degradation limits its use in other applications.

Ko et al. developed an optimized system of microencapsulation of curcumin through $\gamma-\mathrm{Na}^{+}$-PGA microcapsules, using the response surface methodology (RSM) [118]. In this work, the main objective was to increase the efficiency of encapsulation through the study of the best surfactant for the process, being studied the effect of concentration of surfactant, $\gamma-\mathrm{Na}^{+}-\mathrm{PGA}$, and curcumin concentrations. Finally, microencapsulation yield, morphology (Figure 41), and particle-size distribution were evaluated. The results obtained showed that Span 80 significantly increases the encapsulation efficiency (EE), and the ideal microencapsulation process is achieved using $\gamma-\mathrm{Na}^{+}$-PGA $6.05 \%$, curcumin $15.97 \%$, and Span $800.61 \%$ with a high EE\% (74.47 $\pm 0.20 \%$ ). It was concluded that $\gamma-\mathrm{Na}^{+}-\mathrm{PGA}$ may be a viable candidate for the coating of oil-soluble components, having potential for application in the food and drug industry.

\section{(b) Poly(lactic acid)}

Poly(lactic acid) (PLA; Figure 36) is a biodegradable polymer that is derived from renewable resources such as corn starch or sugar cane. Since it is a thermoplastic and highly resistant polymer, it can be manufactured from renewable sources to produce articles for use in the packaging field and in the medical device market. To produce high molecular weight polymers, bacterial fermentation has to take place. This process consists in the production of lactic acid, which is converted into the lactide dimer that serves to remove the water molecule [119-121]. These microcapsules were first introduced in 1979 by Beck et al. [122]. In recent years, several groups of researchers have reported the preparation of polylactide microcapsules containing different drugs and studied the effects of manufacturing parameters on the microcapsule's characteristics. 


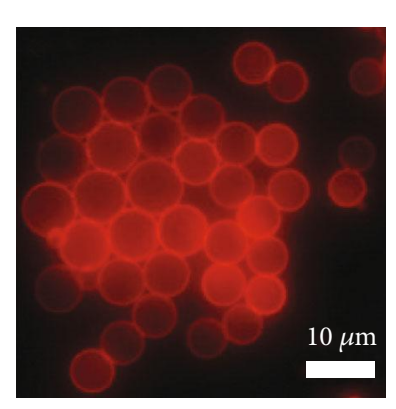

(a)

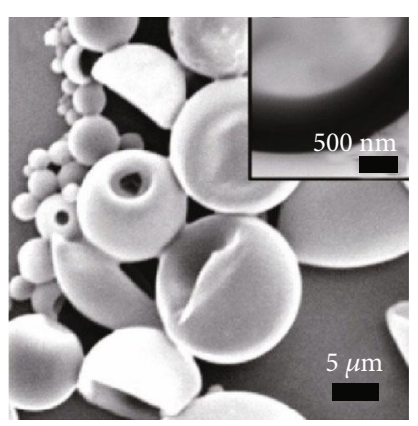

(b)

FIGURE 37: (a) Fluorescence microscopy and (b) SEM and TEM (inset) micrographs of PDLLA microcapsules labeled with 1 wt\% Nile Red relative to polymer. The microcapsules were obtained through evaporation of organic solvent from emulsion droplets containing $5 \mathrm{mg} / \mathrm{mL}$ PDLLA and $3 \mathrm{mg} / \mathrm{mL}$ Aerosol OT [115].

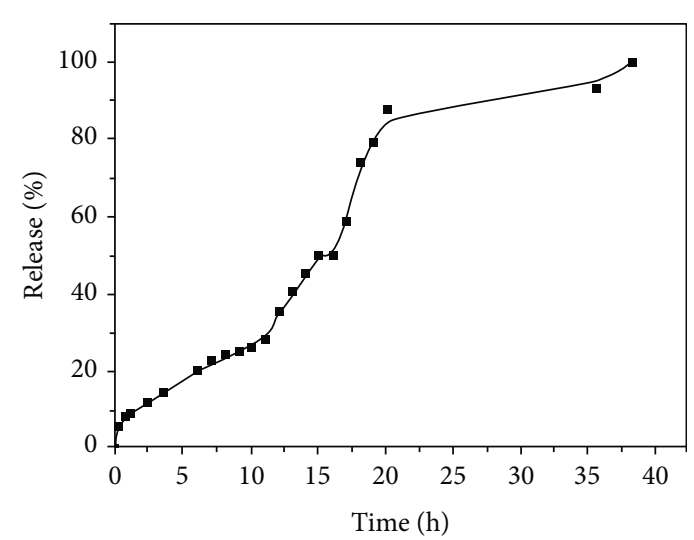

Figure 38: Plot of release of encapsulated Congo Red from PMMA microcapsules [115].

In the study reported by Chronaki et al., microcapsules of PLA of different molecular weights were created through solid-state polymerization (SSP), evaluating the efficiency of this technique as a postencapsulation modification step [123]. Initially, PLA microcapsules were prepared by double emulsion-solvent evaporation and then subjected to SSP in a fixed bed reactor under nitrogen flow, causing postpolymerization reactions in the polymeric walls. In addition, SSP also served as a postcrystallization step that allowed for greater crystallinity and melting temperature.

An increase in the molecular weight of microcapsules of almost $70 \%$ and an increase in crystallinity of up to $60 \%$ were obtained. However, the total spherical topology of microcapsules was not maintained due to the aggregation of particles. SEM micrographs (Figure 42) showed that the surface of microcapsules is smooth, which can be attributed to the emulsification step. In addition, the presence of holes that could arise due to burst solvent escape during the solidification process is not observed.

In this study, it was concluded that it is possible to adapt SSP process from the conventional mass polymer scale to the microcapsule geometry scale in a viable way, avoiding the disadvantages of conventional encapsulation step and giving the possibility of producing customized application products.
(2) Polycaprolactone. Polycaprolactone (PCL; Figure 36) is a biodegradable polyester that is used in the production of special polyurethanes and used as an additive to resins, to improve its processing characteristics and properties for end use. PCL provides good resistance to water, oil, solvent, and chlorine to the produced polyurethane.

Several researchers have studied PCL as a substrate for biodegradation [124-127] and as a matrix in controlled drug delivery systems [128-130]. It is degraded by the hydrolysis of its ester bonds under physiological conditions, therefore, has received a lot of attention for its use as an implantable biomaterial. A variety of drugs have been encapsulated within the PCL spheres for controlled release and targeted drug delivery.

Polyesters derived from alkenediols and alkanedicarboxylic acids are easily degraded by biological systems [131], with some limitations related to their low molecular weight and poor physical resistance.

There are two methods for synthesizing PCL, namely, by polycondensation and by ring-opening polymerization (ROP). In first method, condensation of 6-hydroxyhexanoic acid takes place under vacuum. The water produced during the reaction is removed continuously. In caprolactone ROP, metals, and organometallic catalysts, rare earth metals and transition metals are used, being stannous octoate the most widely used chemical catalyst for the polymerization of caprolactone [132]. This method is the mainly used, making it preferred for PCL synthesis.

In 2009, Petitti et al. proposed a controlled release of polycaprolactone microcapsules in order to keep vancomycin (a glycopeptide antibiotic) at the desired therapeutic concentration, in the aqueous humour of the eye for a necessary period [133]. A simple in vitro release process (in an aqueous solution and without degradation of the active principle) is considered and through a comparison with the experimental release data, the diffusivity of vancomycin in the coating is determined.

In Figure 43, it can be observed that the release curves predicted for the two approaches are very close, almost overlapping. Considering the simplest approach of a single class, the model was applied to the problem of interest 


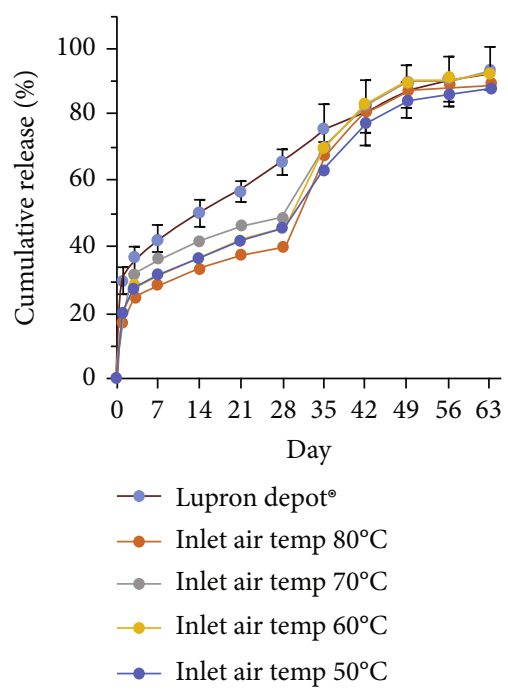

(a)

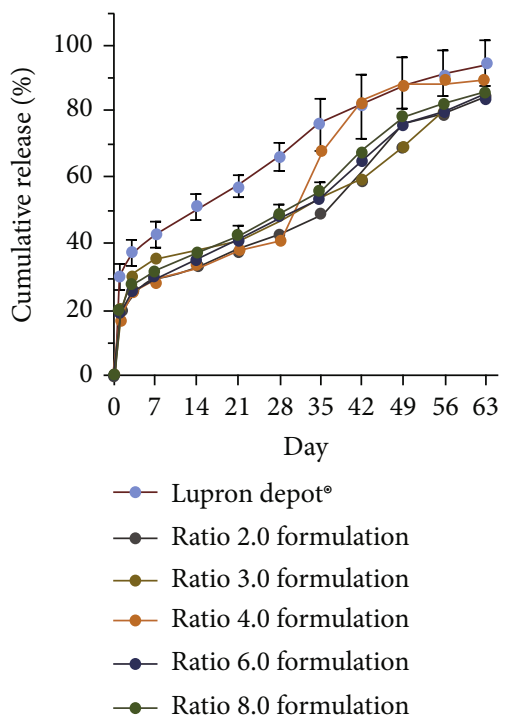

(d)

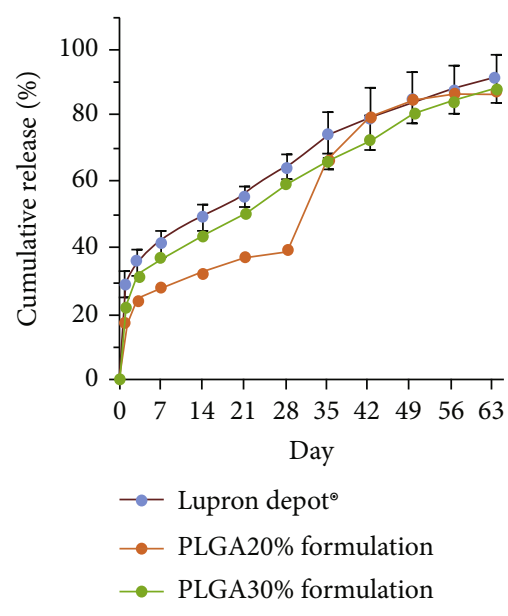

(b)

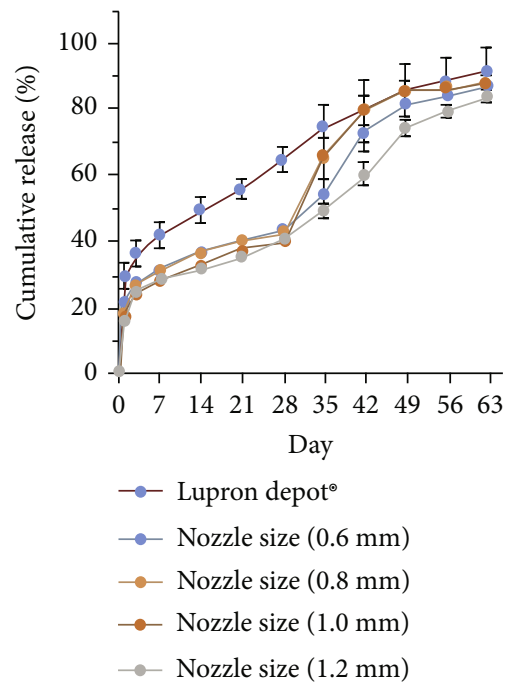

(e)

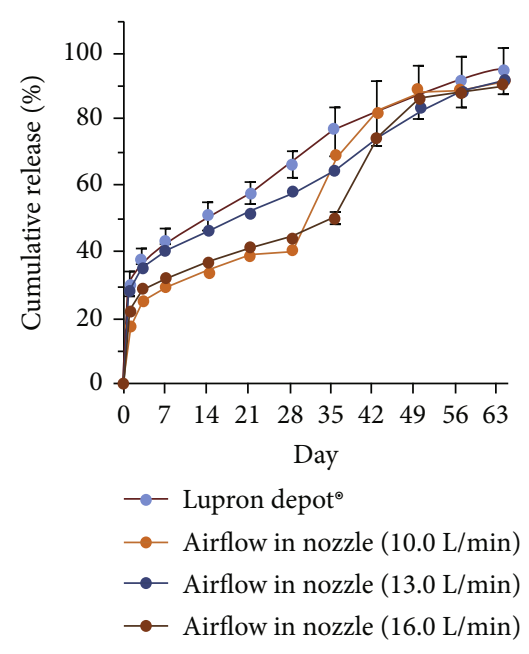

(c)

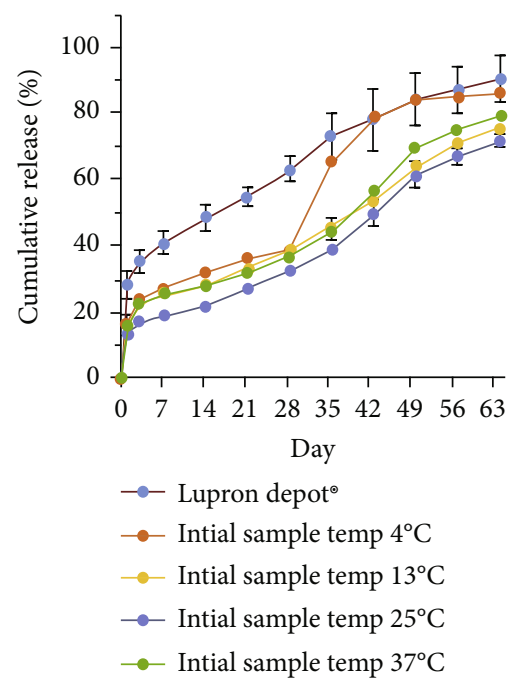

(f)

Figure 39: Cumulative release of leuprolide from all formulations of spray-dried microspheres and the Lupron Depot ${ }^{\circledR}$ (LD). Microspheres were incubated in PBST ( $\mathrm{pH} 7.4$ ) at $37^{\circ} \mathrm{C}$. Panels are grouped according to formulations with varied spray drying parameters, including inlet air temperature $\left(T_{\text {inlet }}, \mathrm{a}\right)$, PLGA concentration $\left(C_{\text {feed }}, \mathrm{b}\right)$, airflow in nozzle $\left(\mathrm{AF}_{\text {nozzle }}, \mathrm{c}\right)$, ratio between atomization gas flow rate and the liquid mass flow rate $\left(\right.$ Ratio $\left._{A / L}, \mathrm{~d}\right)$, nozzle size $\left(S_{\text {nozzle }}, \mathrm{e}\right)$, and initial sample temperature $\left(T_{\text {feed }}, \mathrm{f}\right)$. The basic formulation (orange symbols) and LD are listed in each panel as controls. Symbols represent mean \pm S.D $(n=3[116])$.<smiles>COC(=O)COC(C)C</smiles>

FIgURe 40: PGA chemical structure.

in vivo: the number of microcapsules was evaluated, which must be present in the ocular solution to guarantee the desired coverage (concentration greater than $20 \mathrm{~g} / \mathrm{mL}$ over a period 48 hours).

Cesari et al. obtained PCL microcapsules with the objective of encapsulating signal molecules, such as citric acid and naringin, an organic acid, and a flavonoid. This encapsulation was motivated to maintain the stability and activity of these signal molecules and to allow slow or controlled release by a period of time, selected according to the needs of plants (Figure 44) [134]. This study made it possible to manufacture intelligent materials for sustainable agricultural practices.

Figure 45 shows the SEM micrographs of polycaprolactone microcapsules obtained with different degrees of magnification. The microcapsules have a spherical shape, a morphology of the nucleus wall, and no significant aggregation. However, most of the samples showed some pores in the surface, being in samples I and J less notorious. Microcapsules that were synthesized using the PCL of $30,000 \mathrm{Mw}$ (Figures 45(a), 45(b), 45(k) and 45(l)), are the ones with 


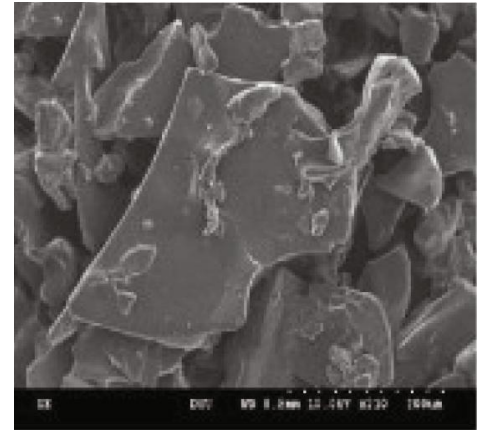

(a)

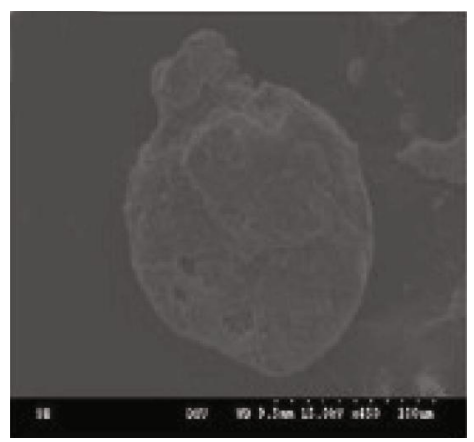

(b)

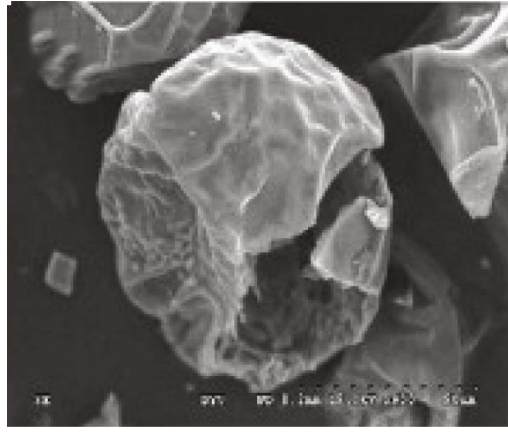

(c)

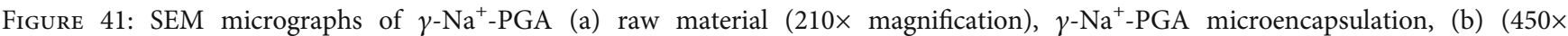
magnification), and (c) (900× magnification) [118].

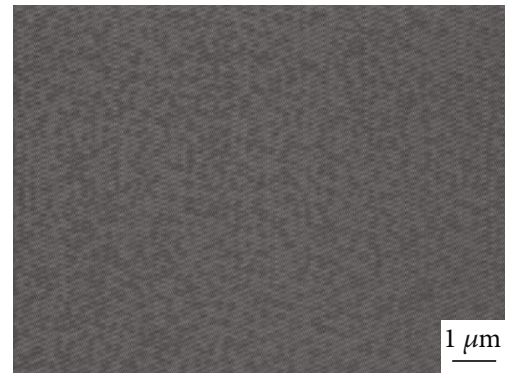

(a)

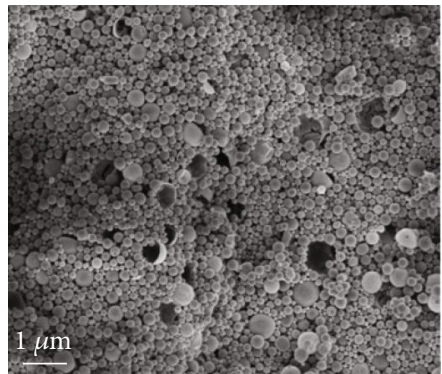

(b)

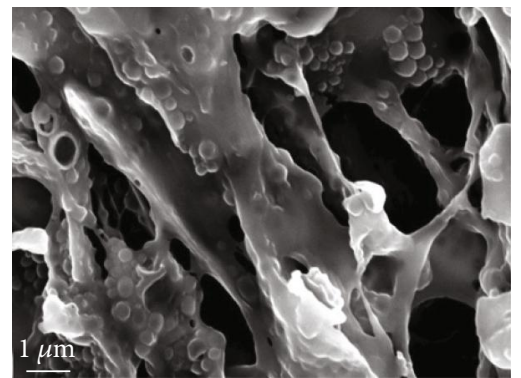

(c)

Figure 42: Microscopy images for MC4 (a) optical microscopy after washing the PVA (400× magnification), (b) SEM after washing the PVA (5000× magnification), and (c) SEM without washing the PVA (2300× magnification) [123].

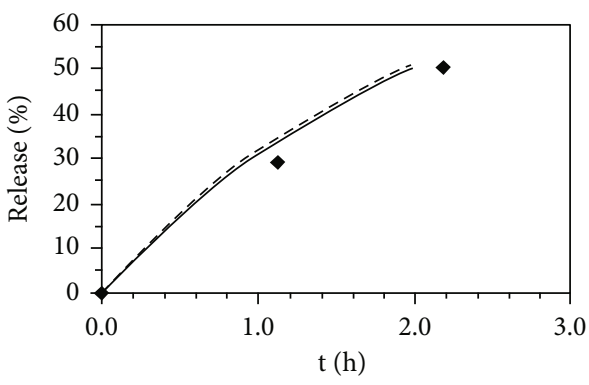

FIgURE 43: Release curve of the drug delivered by $1 \mathrm{~g}$ of microcapsules, in $80 \mathrm{~mL}$ of external solution, perfectly mixed, where the process of drug degradation is absent. The diamond symbols represent the experimental data, the dotted line the release predicted by the model that considers a unique class of particles characterized by average properties, and the continuous line the release predicted by the model that uses distinct classes to represent the particle size distribution [133].

more pores in the surface, being that it offers less resistance to the diffusion of water, in comparison with higher PCL of Mw. When using 45,000 Mw PCL (Figures 45(c)-45(f) and $45(\mathrm{~m})-45(\mathrm{p}))$, the microcapsules have a smoother surface when compared to microcapsules with $80,000 \mathrm{Mw}$ PCL (which have a thicker wall, (Figures 45(g)-45(j) and 45(q)45(t)). In Figures 45(l) and 45(p)), the presence of naringin in crystalline form can be observed.
The cumulative release profile of citric acid and naringin from the PCL microcapsules to the aqueous medium at different $\mathrm{pH}$ values $(4,5,7)$ and to Arachis hypogaea $\mathrm{RE}(\mathrm{pH} 5)$ is represented in Figure 46. As can be seen, the error bars are small, thus, confirming that the same type of release behaviour is obtained for different batches of microcapsules synthesized following the same procedure.

For microcapsules $\mathrm{M} 30(16) \mathrm{C}(80)$ (prepared with lower molecular weight (Mw) of PCL), it is observed that there is a rapid release of citric acid after 3 days, being faster at pH 4 and 5. After 15 days, the citric acid content was released in $100 \%$, both in aqueous solutions and in plant root exudates (RE) (Figure 46(a)). Furthermore, it was concluded that a thinner shell and surface holes can lead to a total encapsulated content release. This characteristic can be observed in the microcapsules obtained for PCL with $30,000 \mathrm{Mw}$. The release of citric acid from M45(16)C(80) was $\mathrm{pH}$ dependent: $93 \%$ at $\mathrm{pH} 4$ after 18 days, $78 \%$ at $\mathrm{pH} 5$ after 40 days, and $67 \%$ at $\mathrm{pH} 7$ after 43 days. When the microcapsules were placed in root exudate, the release of citric acid was 35\% after 45 days (Figure 46(b)). It can be seen in Figure 46(c) that with $80,000 \mathrm{Mw}$ PCL microcapsules, the release of citric acid was $70 \%$ at $\mathrm{pH} 4-5$ and $28 \%$ at $\mathrm{pH} 7$.

Figures 46(d)-46(f) show the naringin release profile of PCL microcapsules in aqueous solutions with different $\mathrm{pH}$ solutions. The release of naringin from the microcapsules was considered independent of $\mathrm{pH}$ of the medium, not 


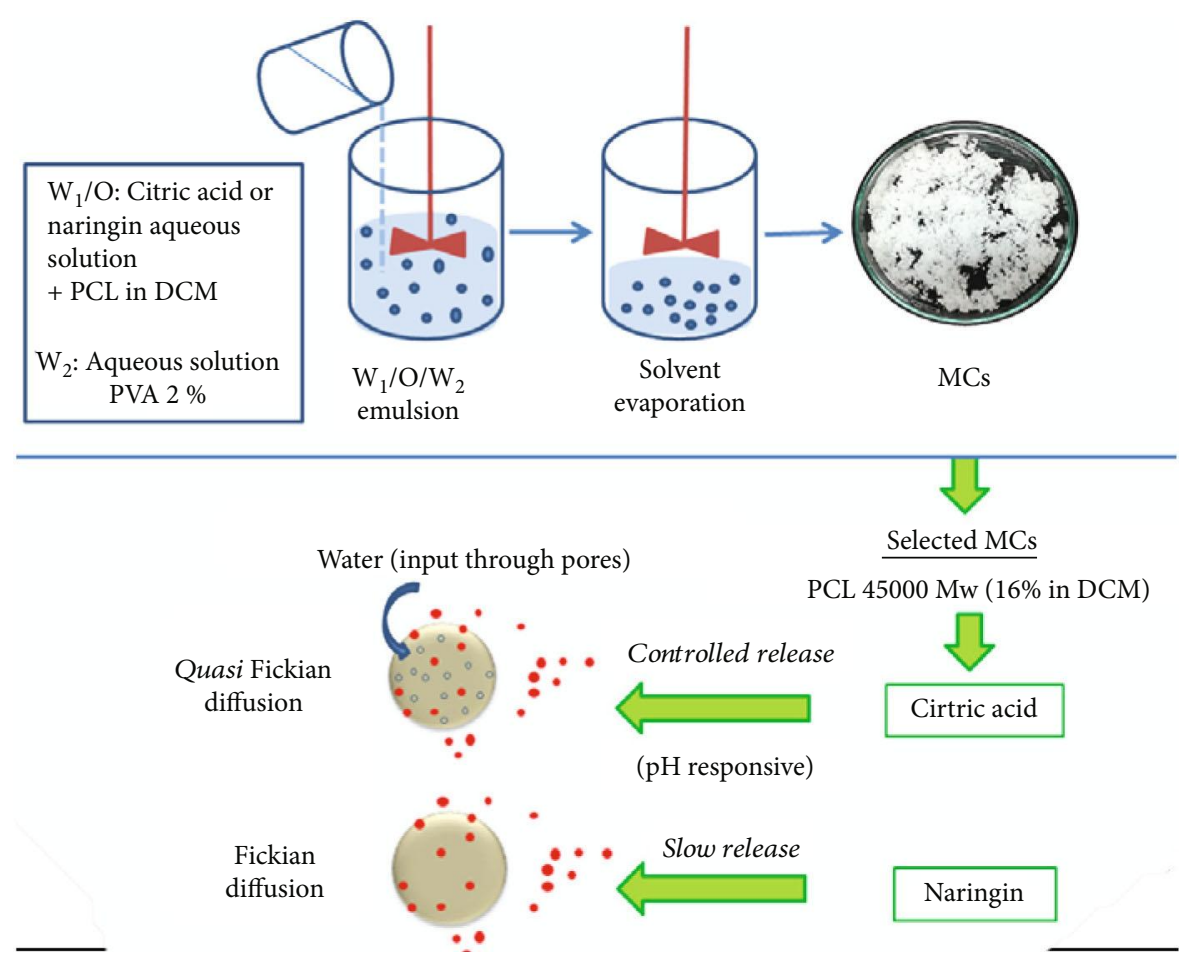

FIGURE 44: Citric acid and naringin microencapsulation: new strategy for sustainable agriculture [134].

having been verified for the release of citric acid. Similar to M30(16)C(80) microcapsules, M30(16)N(5) microcapsules showed rapid release after 4 days in aqueous solutions with total release. When placed in $\mathrm{RE}$, naringin release reached $80 \%$ after 5 days. On the other hand, M45(16)N(5) showed a gradual release of naringin over 30 days, being slightly higher at $\mathrm{pH} 4$ and 5 than at $\mathrm{pH}$. When placed in $\mathrm{RE}$, the release was $80 \%$, as can be seen for $\mathrm{pH} 7$. For $\mathrm{M} 80(12) \mathrm{N}(5)$, the release of naringin was gradual, slow, and also independent of the $\mathrm{pH}$ of the medium, showing a release of $32 \%$ after 8 days when placed in RE. There is also a gradual release reaching $67 \%$ after 45 days.

In this case, the release of naringin by the microcapsules depends on the molecular weight of the PCL, being the reason why it was slower and more gradual in the microcapsules with a molecular weight of 45.000 and 80.000 , showing a dependence on the morphology of microcapsules and characteristics in the shell.

(3) Polyamides. Polyamide is a macromolecule with repeated units linked by amide bonds that can occur naturally and artificially. Proteins, such as wool and thirst, are considered natural polyamides, whereas artificial polyamides can be produced through polymerization, producing materials such as nylon (Figure 47). These artificial polyamides are normally used in automotive industry and textiles as sportswear, due to their high durability and resistance [135].

The biodegradation of polyamides is low, and these materials can often be considered nondegradable. However, they can be degraded through enzymes and microorganisms of low molecular weight [136-140]. The introduction of sub- stituents such as benzyl, hydroxy, and methyl greatly improve the biodegradation.

There is a lesser tendency for natural proteins to become involved in highly ordered morphologies, which makes them accessible to enzymatic attack, since the natural proteins rarely contain repeated units. With synthetic polyamides, this phenomenon is not verified. These polymers have short and regular repetition units, high symmetries, and strong hydrogen bond between chains, which result in crystalline morphologies, limiting accessibility to the enzymatic attack. However, it has been found that certain polymers with long repetition chains are degraded at rates similar to synthetic polyamides [141].

Wang et al. developed "liquid core" polyamide microcapsules using an interfacial polymerization between diester diacid chloride with DETA and a triazine trisamine [142]. The microcapsules formed are stable in a dry or hydrophobic environment, allowing a stable controlled release at $\mathrm{pH} 7.4$ and an accelerated release at $\mathrm{pH} 5$ and $\mathrm{pH} 10$. The release profile can be controlled by adjusting the amine cross-linker feed ratio, which controls the degree of crosslinking in the polymer shell, showing a simple adjustment mechanism.

The release profile is shown in Figure 48. It is possible to observe that in the absence of water, regardless of the ratios of the aqueous monomers, the samples did not show a noticeable release of dye in toluene. On the other hand, all microcapsules that were contracted with aqueous buffer solutions ( $\mathrm{pH} 5,7.4$, and 10) released dye in the toluene phase. This behaviour indicated that exposure to a humid environment promotes the release of encapsulated dye and that it is influenced by the $\mathrm{pH}$ of the environment. In 


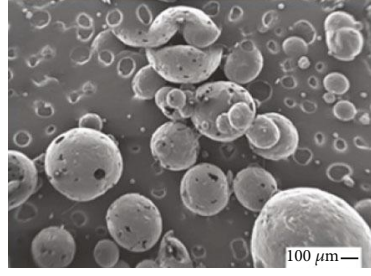

(a)

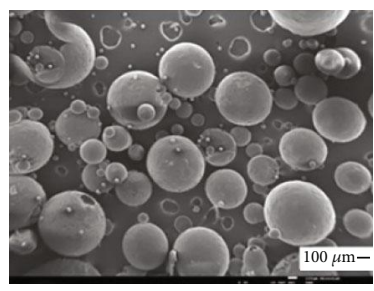

(c)

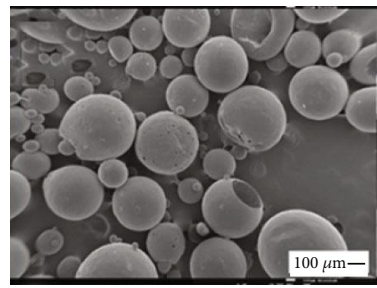

(e)

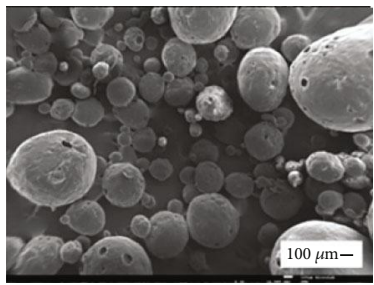

(g)

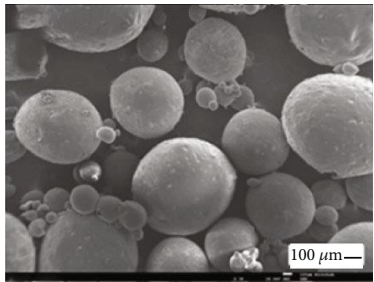

(i)

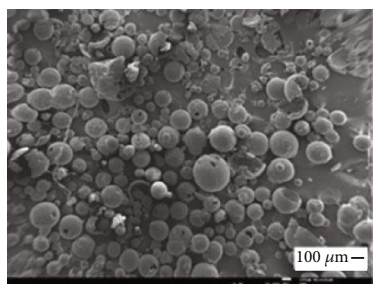

(k)

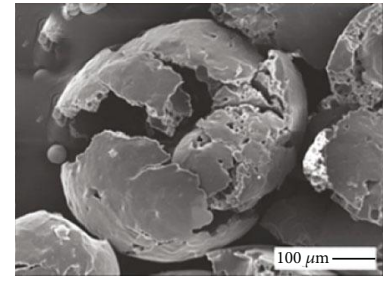

(b)

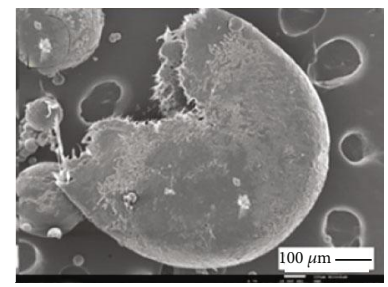

(d)

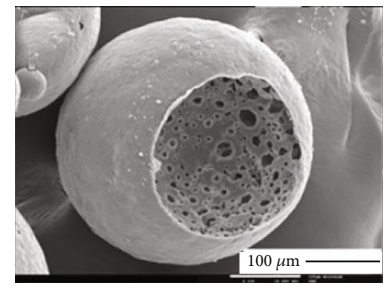

(f)

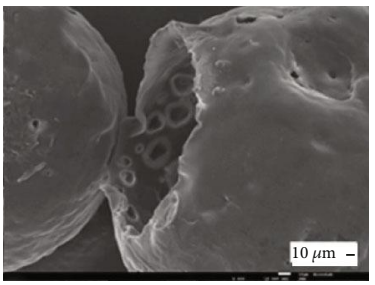

(h)

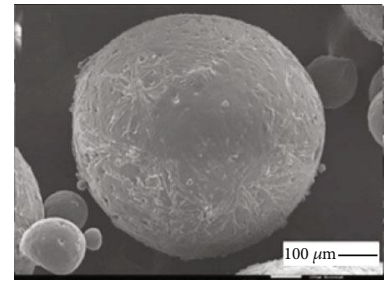

(j)

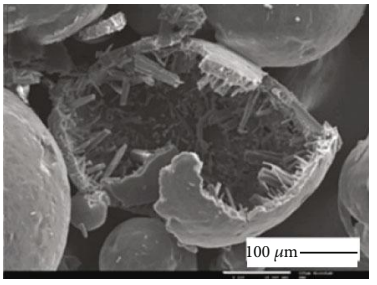

(1)

Figure 45: Continued. 


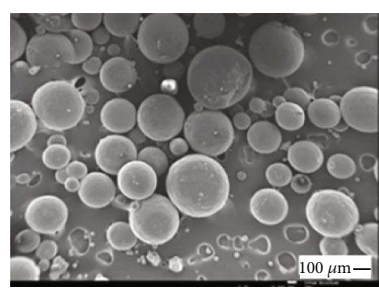

(m)

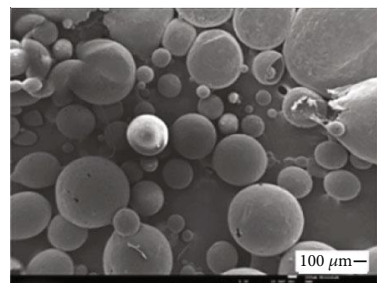

(o)

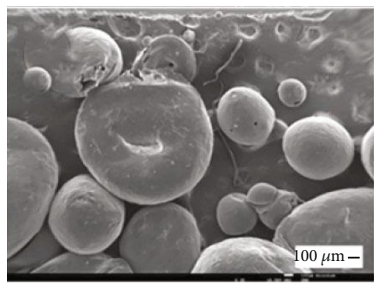

(q)

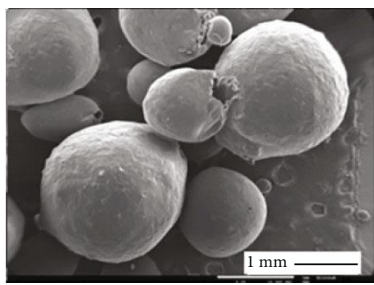

(s)

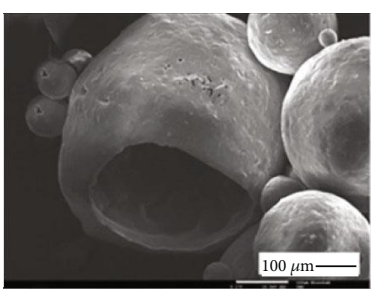

(n)

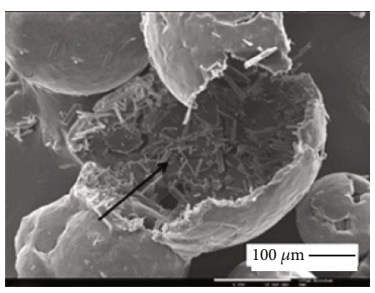

(p)

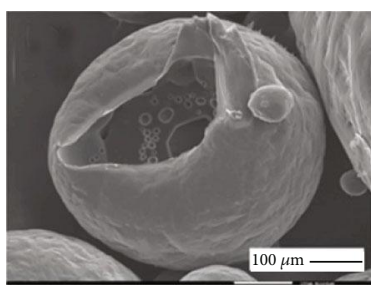

(r)

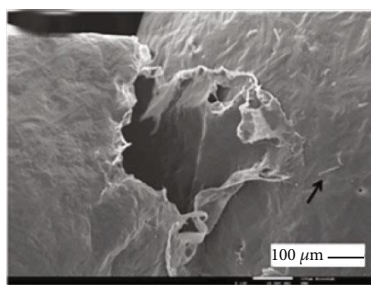

(t)

FIGURE 45: SEM micrographs of the polycaprolactone microcapsules; the arrow in Figure 44(p) indicates the presence of encapsulated naringin crystals. The same happens in Figure 44(l) [134].

contrast, in a dry environment, the microcapsules have a stable behaviour that allows to protect and inhibit the release of encapsulated dye.

In short, the thin-shell microcapsule formed has adequate protective properties but allows controlled release triggered by $\mathrm{pH}$ variations. Furthermore, it permits the application in a wide range of $\mathrm{pH}$ environments, and its preparation is simple enough to be applied to a larger scale of production. The response to $\mathrm{pH}$ variation, with different release rates and the ability to retain a volatile core for a long period of time in a nonpolar or dry environment, makes this microencapsulation system suitable for several applications. Some of them are the distribution of pest control agents in the agricultural sector and alkaline washing process or release of fragments on moist skin surfaces.

In another study, microcapsules were developed for the first time with the shell completely constituted by a slightly cross-linked liquid crystalline polymer. First, Tylkowski et al. synthesized a new liquid crystalline polyamide, slightly crosslinked, in which order and conformation could be triggered by external stimuli, such as temperature and light
[143]. Then, microcapsules were prepared with the shell consisting of a polymer synthesized with different contents in the core (toluene, naphthalene, or $\beta$-carotene). By continuous irradiation with ultraviolet light at $364 \mathrm{~nm}$ and at room temperature, it was observed that the polymer underwent E$\mathrm{Z}$ photoisomerization. Through the morphological analysis, it was observed that the external surface of microcapsules seems smooth and dense, while some heterogeneities are visualized in the internal face. Depending on the encapsulated material, the diameter of microcapsule ranges from 30-120 $\mu \mathrm{m}$. In the SEM micrograph of Figure 49, it is shown a fractured microcapsule with $\beta$-carotene in the core.

$\beta$-Carotene release rate was carried out in water and tetrahydrofuran (THF), in the presence and absence of UV radiation $\left(\right.$ at $20^{\circ} \mathrm{C}$ ). The temperature cannot be used to trigger the release of $\beta$-carotene because the microcapsules fractured after 300 minutes at $50^{\circ} \mathrm{C}$, due to the internal pressure of microencapsulated toluene. In water, the release was greatly influenced by UV irradiation (Figure 50). While in the absence of UV irradiation, the release of $\beta$-carotene was minimal (plateau value of about $2.5 \%$ after a few minutes), when subjected to 


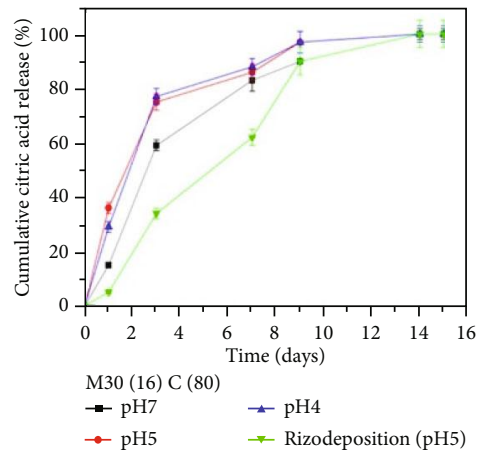

(a)

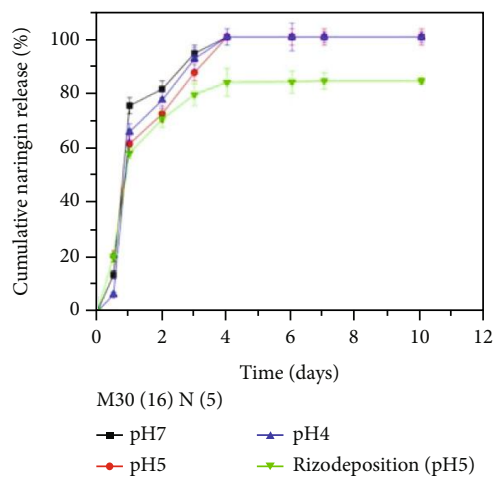

(d)

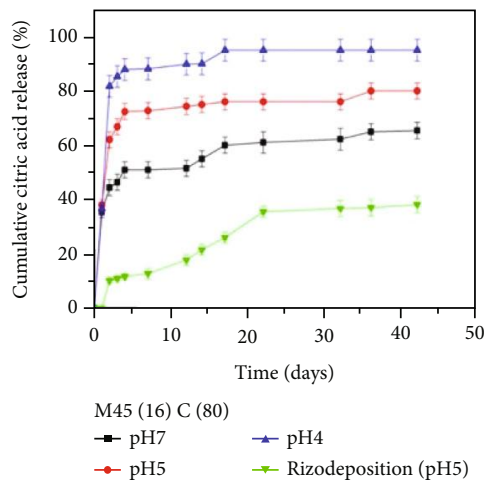

(b)

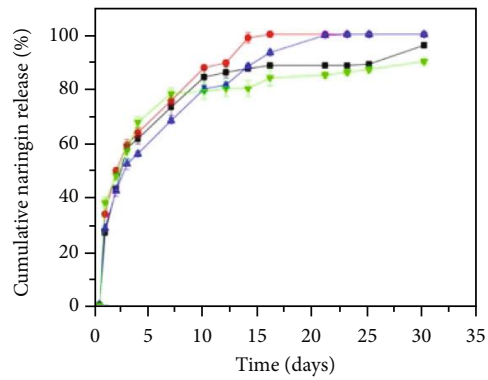

$$
\begin{array}{ll}
\mathrm{M} 45(16) \mathrm{N}(5) & \\
-\mathrm{pH} 7 & \neq \mathrm{pH} 4 \\
-\mathrm{pH} 5 & \rightarrow \text { Rizodeposition (pH5) }
\end{array}
$$

(e)

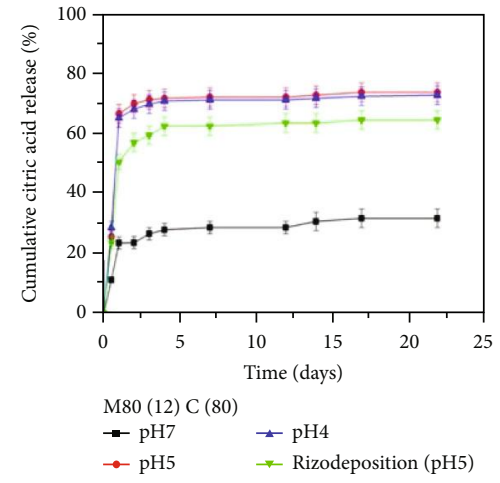

(c)

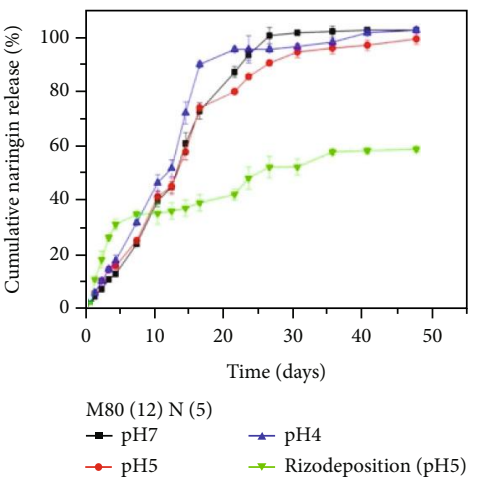

(f)

Figure 46: Percentage of cumulative in vitro release profiles of (a) microcapsules containing citric acid: $\mathrm{M} 30(16) \mathrm{C}(80)$, M45(16)C(80), and M80(12)C(80); (b) microcapsules containing naringin: $\mathrm{M} 30(16) \mathrm{N}(5), \mathrm{M} 45(16) \mathrm{N}(5)$, and M80(12)N(5), under different pH solutions. The release values displayed in the graphs are an average of three independent release experiments employing microcapsules of the same type, but from different batches (error bars represent standard deviations, $n=3$ ) [134].<smiles>N[R]PN</smiles><smiles>CCCC(C)N[R]NC(=O)[R]C(=O)C(C)(C)C</smiles>

FIgURE 47: An example of a polyamide reaction.

continuous irradiation, $\beta$-carotene was released quickly and released in full after 5 minutes. In THF, $\beta$-carotene was released in full after 40 minutes (Figure 51).

Based on these results, it can be concluded that albeit the study is preliminary, considering that it is the first development of microcapsules with shells entirely constituted of cross-linked liquid crystalline polymer, it has potential. Through the optimization of conditions such as state of order of the shell, thermal stability, and structural changes that occur through UV irradiation, this application of developed microcapsules has prospects.

(4) Polyurethanes and Polyureas. Polyurethane (PU) is composed of organic units joined by carbamate (urethane) bonds, with some polyurethanes being thermosetting. This polymer can be used in buildings insulation, construction, transportation, packaging, fabrics, footwear, and medical products [144]

PU is a versatile polymer because its physical and chemical properties can be widely adapted to meet the demands of modern technologies. Hence, there are no defined properties for polyurethanes. The best-known properties are resistance to extreme temperatures, which will not cause its degradation, and are good electrical insulators. Regarding the biodegradation properties of polyurethanes, they show to be dependent on the prepolymer (polyisocyanate, polyester, or polyether) [145].

The preparation of polyurethane (Figure 52) is achieved through condensation and addition reactions. Addition reaction is done by adding a diisocyanate with a diol [146]. This polymer can be soft (foams) or hard (automotive parts). The rate of degradation increases with increasing length of polyester segment, which happens when a series of polyurethanes with various molecular weights and aliphatic or 


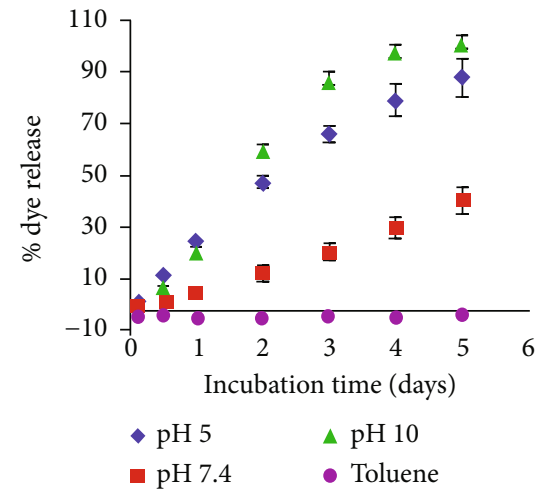

(a)

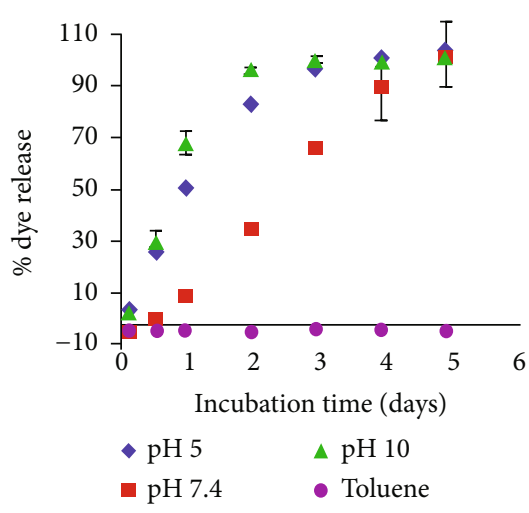

(b)

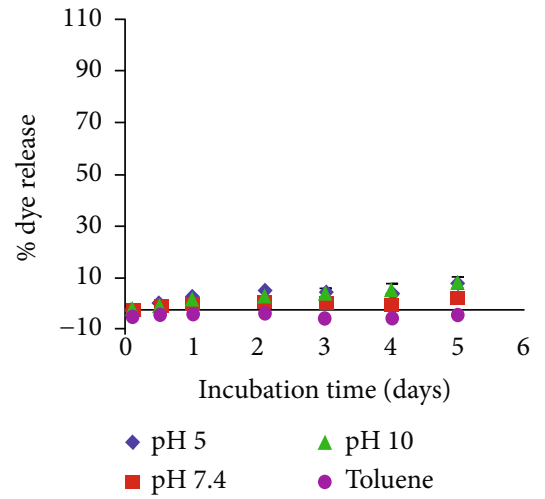

(c)

FIGURE 48: Release of coumarin 1 dye under different solvent conditions for polyamide capsules prepared with (a) $3: 1$ triazine 2:DETA and (b) 1:1 triazine 2: DETA. (c) Control experiments: 1:1 triazine 2: DETA with terephthaloyl chloride. Uncertainty represents standard deviation of three measurements [142].

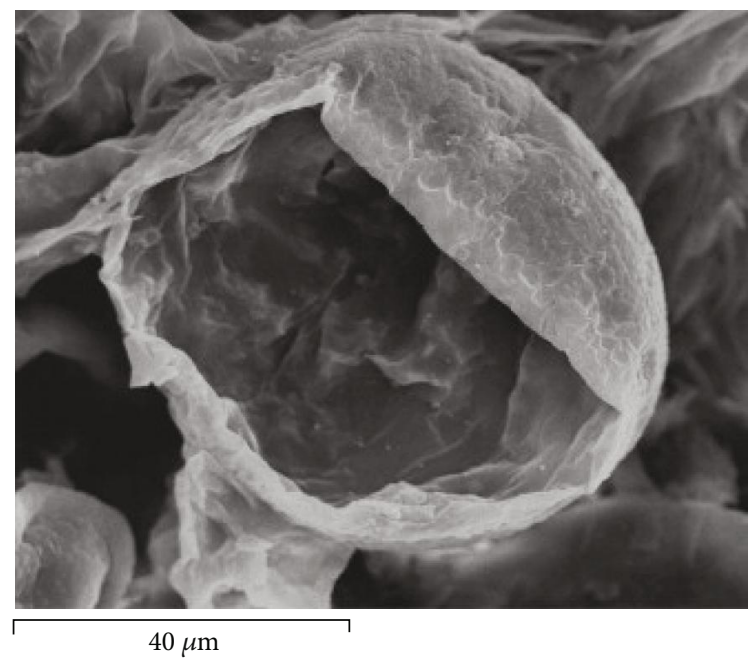

FiguRE 49: SEM micrograph of a fractured microcapsule having $\beta$ carotene as a core [143].

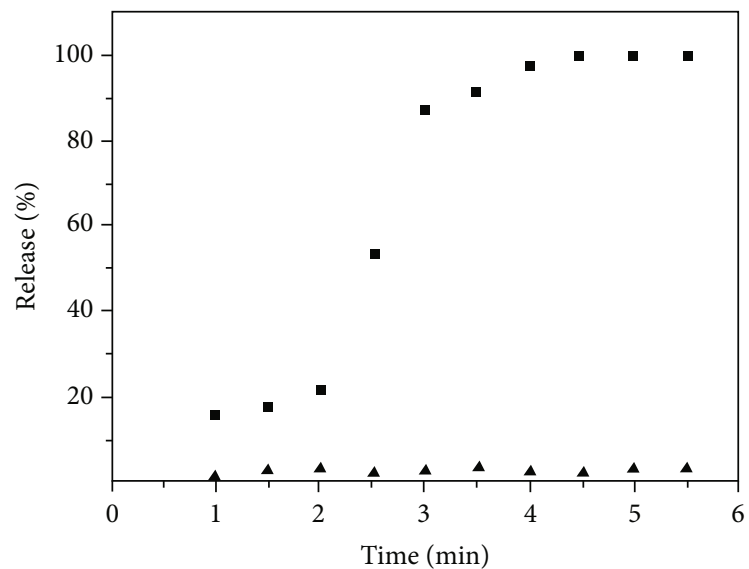

FIGURE 50: Release kinetics of $\beta$-carotene from polyamide microcapsules in water at $20^{\circ} \mathrm{C}$ in the absence (triangular symbols) and in the presence (square symbols) of continuous irradiation with UV light [143].

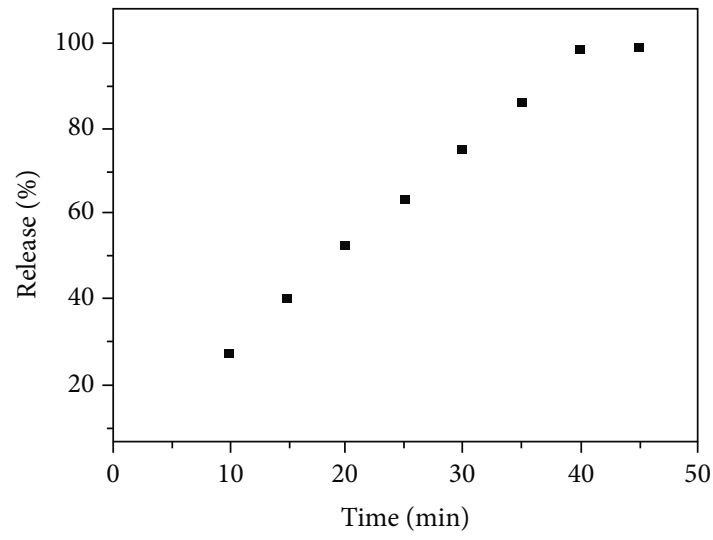

FIgURE 51: Release kinetics of $\beta$-carotene from polyamide microcapsules in tetrahydrofuran at $20^{\circ} \mathrm{C}$ [143].

aromatic diisocyanates are treated with several organisms. It has also been found that polyurethanes derived from aliphatic diisocyanates are degraded more rapidly than those derived from aromatic diisocyanates [147].

In the field of research, many scientists have focused on encapsulating polyurethane. In 2015, isophorone diisocyanate (IPDI) monomer as an active agent was investigated via interfacial polymerization in a stabilized aqueous emulsion [148]. The authors reported studies on the possibility of 1,4-butandiol, 1,6-hexanediol, and glycerol as polyol monomers for polyurethane microcapsule shell. In the latter work, PU microcapsules were characterized using different methods. Morphological analysis and the size of microcapsules containing IPDI investigated by SEM are shown in Figure 53. The microcapsules are spherical with a tendency to agglomerate, and this fact is associated with the presence of isocyanate that did not react completely during air drying of microcapsule.

Liu et al. studied spherical and disk polyurethane microcapsules that were prepared by an adsorption and crosslinking method in models with corresponding forms in organic solvent (Figure 54) [149]. The internalization 


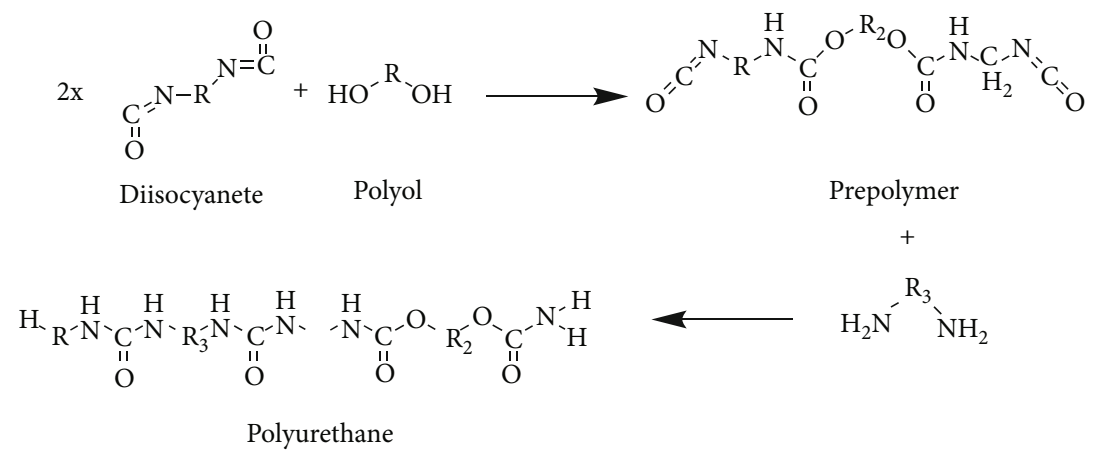

FIGURE 52: Synthetic pathways of polyurethane.

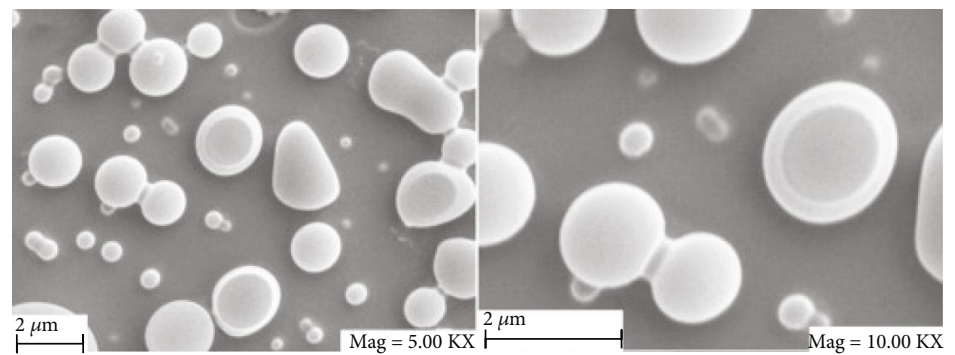

(a)

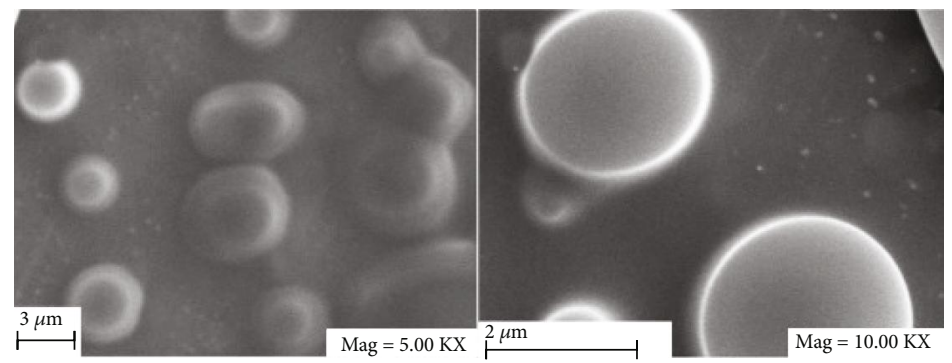

(b)

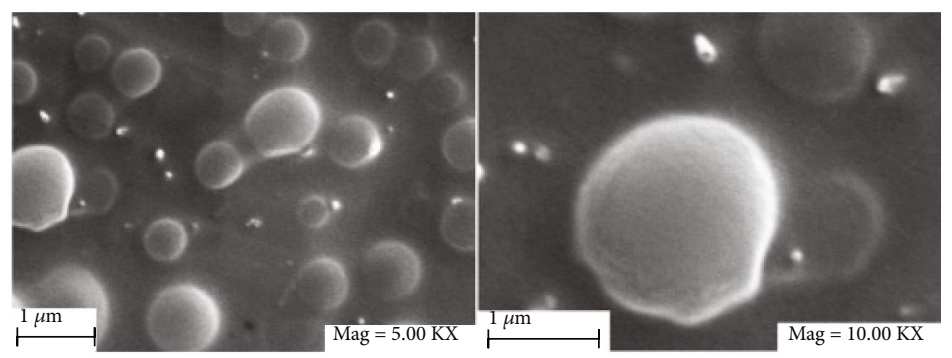

(c)

FIGURE 53: Morphology and size of the PU microcapsules synthesized using (a) 1,4-butanediol, (b) 1,6-hexanediol, and (c) glycerol [148].

behaviours of microcapsules were investigated by coincubation with RAW 264.7 and HepG2 cells.

More recently, polyurethane microcapsules were prepared using combined method of interfacial polymerization with the Peckering emulsion modelling method (Figure 55) [150].

[BMIm]PF6@PU (polyurethane (PU) microcapsules containing 1-butyl-3-methylimidazolium hexafluorophosphate ([BMIm]PF6)) microcapsules were synthesized at different emulsification rates and with different levels of lignin (Table 2, samples 1-4). As can be seen in Table 2, the average particle size of microcapsules decreased significantly when the emulsification stirring speed was increased, while keeping the concentration of emulsion constant. A conclusion to be drawn is that the higher the stirring speed of emulsification, the greater the shear strength and the lower the average particle size of produced microcapsules.

From observing Figure 56(a), for a slower emulsification rate $(400 \mathrm{rpm})$, the size of microcapsules is larger, and most of the microcapsules are coalesced. A lower emulsification rate is not effective in dispersing highly viscous ionic liquids. 


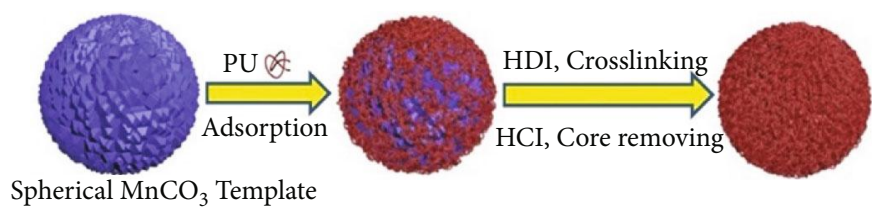

(a)

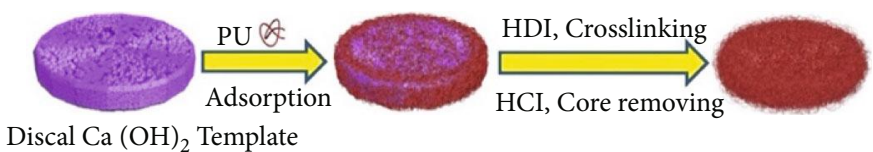

(b)

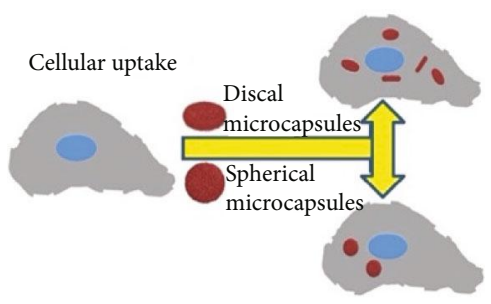

(c)

FIGURE 54: Fabrication process of (a) spherical and (b) discal PU microcapsules and (c) the cellular uptake of the microcapsules [149].

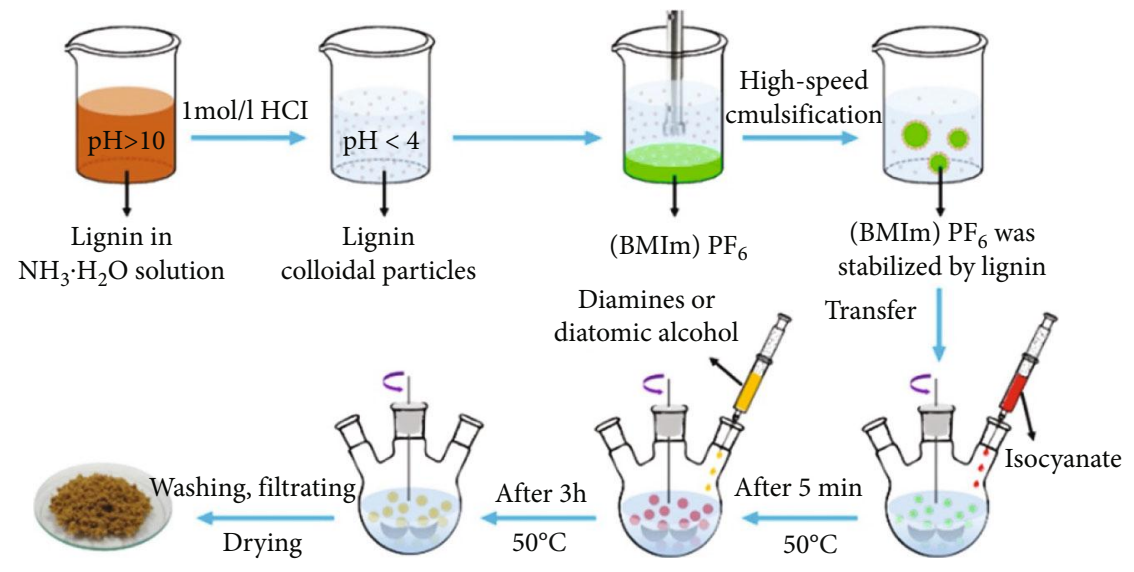

FIGURE 55: Synthetic process of PU microcapsules by the interfacial polymerization in lignin stabilized Pickering emulsion [150].

TABLE 2: Mean diameter of [BMIm]PF6@PU microcapsules obtained with different emulsification rate and with different lignin content, adapted from [150].

\begin{tabular}{lccccc}
\hline Sample & Lignin content (wt.\%) & Emulsification mode & $\begin{array}{l}\text { Emulsification conditions } \\
\text { Emulsification time }(\mathrm{min})\end{array}$ & Emulsification rate $(\mathrm{rpm})$ & Mean diameter $(\mu \mathrm{m})$ \\
\hline 1 & 0.5 & Mechanical agitation & 30 & 500 & $100 \pm 30$ \\
2 & 0.5 & High speed emulsification & 5 & 2.000 & $90 \pm 20$ \\
3 & 0.5 & High speed emulsification & 5 & 5.000 & $40 \pm 20$ \\
4 & 0.5 & High speed emulsification & 5 & 8.000 & $30 \pm 10$ \\
\hline
\end{tabular}

By increasing the emulsification rate, the adhesion between microcapsules is reduced, making the spherical structure more regular and sizes more uniform (Figures 56(b) and $56(\mathrm{c})$ ). The best result was achieved for an emulsification rate of $5000 \mathrm{rpm}$; for $8000 \mathrm{rpm}$ the size of microcapsules further decreased even. However, some of microcapsules were fractured at $8000 \mathrm{rpm}$ (Figure 56(d)).
He et al. prepared polyurethane (PU) microcapsules containing pirimiphos-methyl (PM) using the interfacial reaction method between toluene diisocyanate (TDI) trimer and 1,4-butanediol [151]. Microcapsules with different corewall relationships were produced and characterized using several techniques. The results showed that PM was encapsulated in PU microcapsules, and through scanning electron 


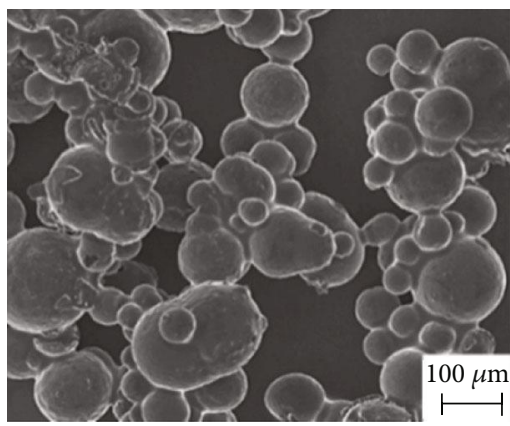

(a)

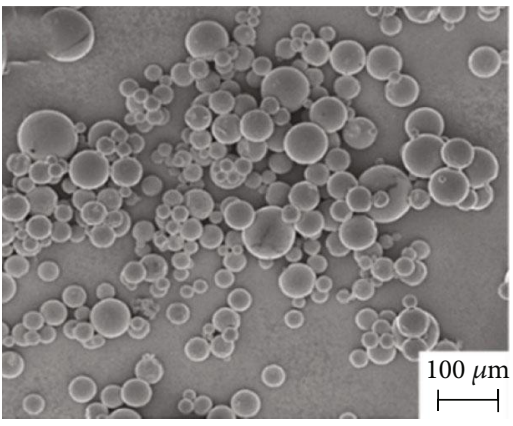

(c)

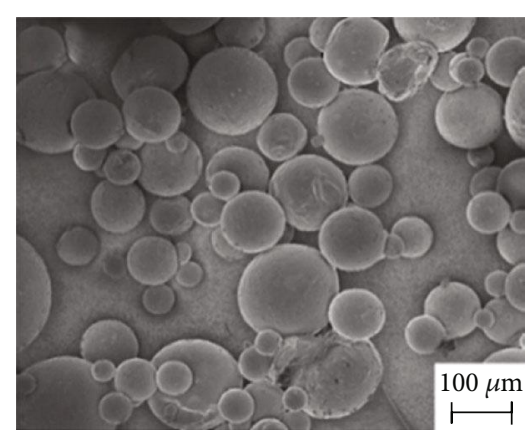

(b)

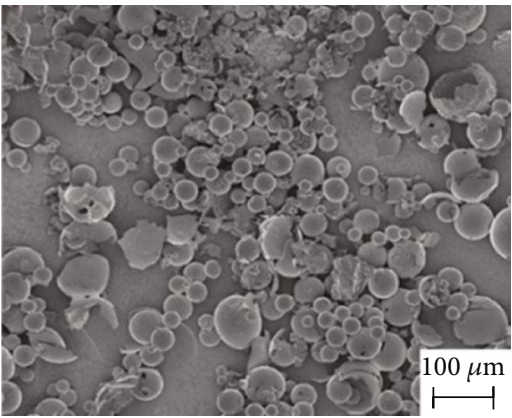

(d)

FIGURE 56: SEM morphologies of [BMIm]PF6@PU microcapsules prepared with increasing emulsification rate (sample 1-4). (a) 500 rpm. (b) $2000 \mathrm{rpm}$. (c) $5000 \mathrm{rpm}$, (d) $8000 \mathrm{rpm}$ [150].

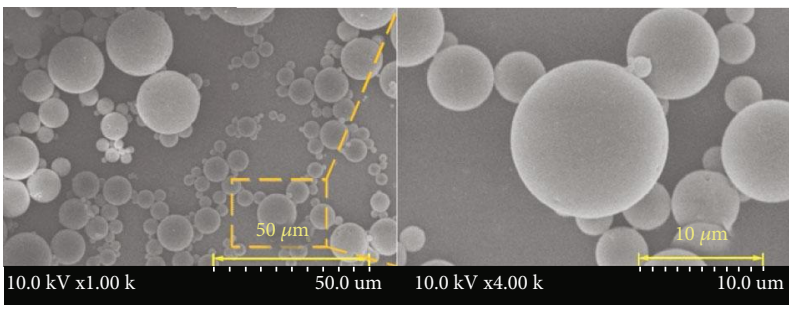

(a)

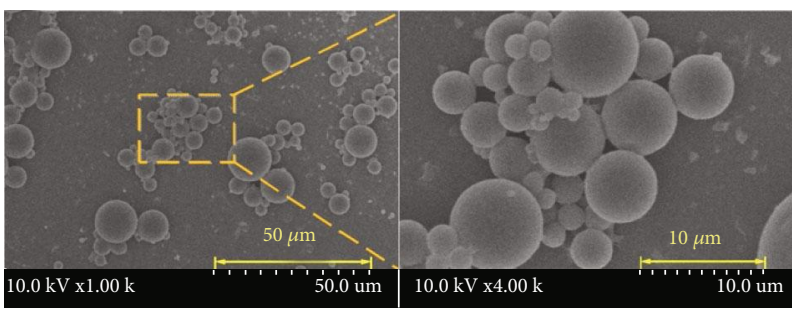

(c)

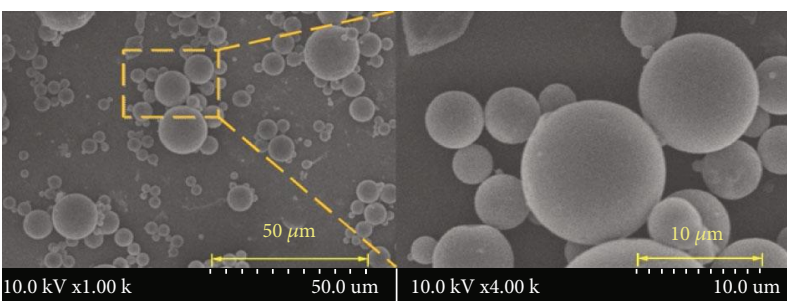

(b)

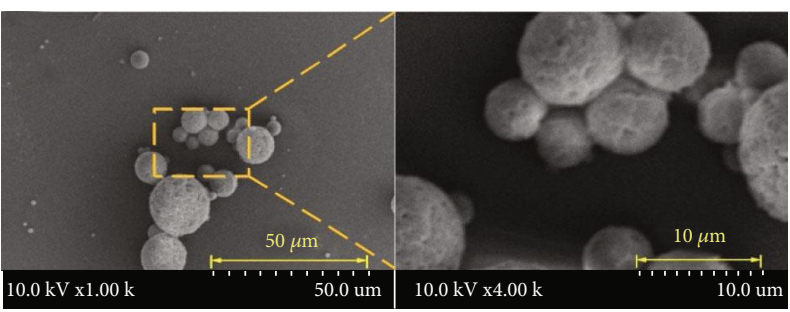

(d)

Figure 57: SEM micrographs of PU microcapsules: (a) S1, (b) S2, (c) S3, and (d) S4 [151].

microscopy, it was showed that microcapsules had a clean surface and a spherical shape (Figure 57). By increasing the proportion of nucleus wall, the surface of microcapsules became wrinkled, and there was an appearance of small pores. It was also verified that the microcapsules are able to protect PM against degradation and showed also sustainable release. This microcapsule procedure allows the authors to conclude that PU microcapsules based on TDI trimer are an environmentally friendly alternative, with potential to be developed as a pesticide that saves resources.
Ribeiro et al. studied the functionalization of titanium dioxide nanoparticles on the surface of polyurethane microcapsules as a way to control the release of citronella by solar radiation, allowing the release of a mosquito repellent through a photocatalytic process, without mechanical stress [152]. Using a modified hydrothermal sol-gel method, $\mathrm{TiO}_{2}$ nanoparticles were synthesized with a mesoporous structure. According to the morphology analysis, microcapsules have an almost perfect spherical morphology, with sizes ranging from 50 to $250 \mu \mathrm{m}$ (Figure 58(a)). When these 


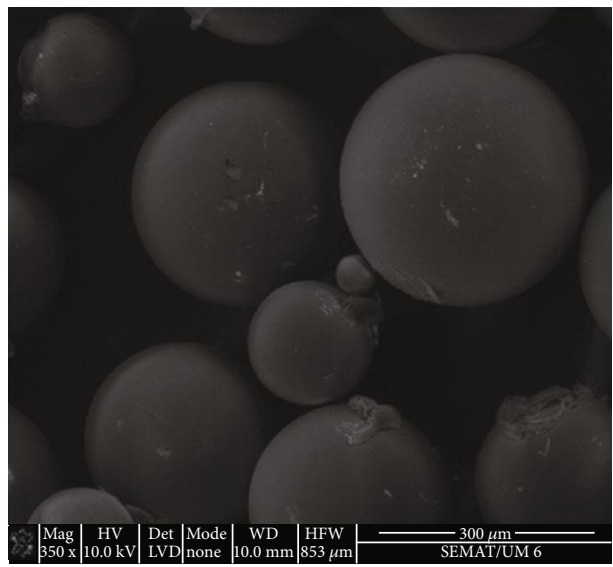

(a)

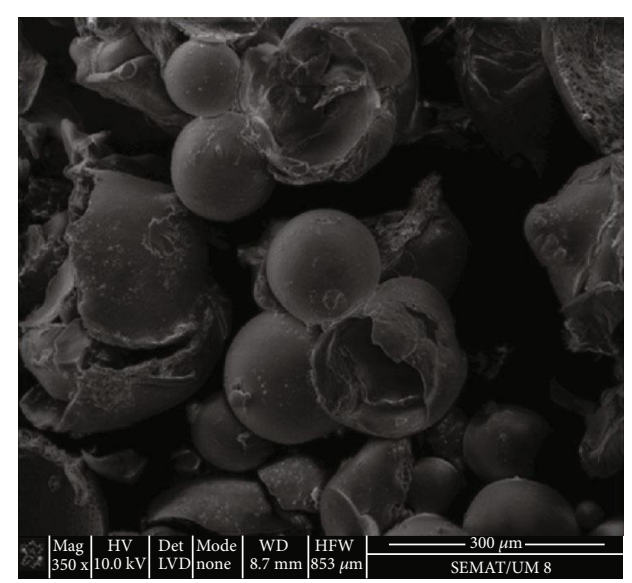

(b)

FiguRE 58: Scanning electron microscopy micrographs of citronella-loaded polyurethane microcapsules (a) before and (b) after mechanical stress [152].<smiles>COC(C)OC(=O)C(C)OC(C)C(C)OC</smiles>

FIGURE 59: Chemical structure of polyanhydride.

microcapsules were subjected to mechanical forces (Figure 58(b)) a hollow interior, with an average wall thickness of $10 \mu \mathrm{m}$, was revealed [152].

To the authors' knowledge, this technology is very promising due to the high photocatalytic activity of the nanoparticles, which promotes a controlled release of the encapsulated product, combined with good mechanical resistance and general quality of the PU microcapsules.

(5) Polyanhydrides. Polyanhydrides (Figure 59) are a class of biodegradable polymers characterized by repeating units that are connected by anhydride bonds. Due to their degrading nature, they show some interest in biomedical applications, such as drugs transportation to various organs in the human body, including the brain, bones, and eyes.

Polyanhydride can be synthesized from available and low-cost resources and can be manipulated to meet desirable characteristics. Bucher et al. synthesized the first synthetic polyanhydride by treating acetic anhydride with isophthalic or terephthalic acid [153]. There is a difference in polymeric degradation between distinct polyanhydrides structures. Aromatic polyanhydrides presents a lower degradation rate, over a long period of time, while aliphatic polyanhydrides degrade faster, within a few days. Polyanhydride synthesis consists of interfacial condensation, ring-opening polymerization, dehydrochlorination, and dehydrating coupling [154]. Biodegradable polymers with high molecular weights can be produced using coupling agents, such as diacyl chloride and many other acid receptors. The rapid synthesis of polyanhydride is also facilitated by microwaves and dielectric heating.
Regarding the biodegradation of polyanhydride, it involves two complementary processes, namely, degradation and erosion. Degradation refers to the chain cleavage process, in which the polymeric chains are cleaved to form oligomers and finally to form monomers. Erosion designates the loss of material due to the monomers and oligomers that leave the polymer [155]. Polyanhydrides are hydrophobic polymers insoluble in water, which makes it mandatory for these materials to degrade before erosion. As they are hydrophobic polymers, they exhibit an ideal surface erosion, which makes the rate of hydrolytic degradation on the surface to be much faster than the rate of water penetration into the matrix [156].

Over the years, several studies have been carried out that describe polyanhydride a new polymer with excellent film and fiber formation properties, structures, degradation and stability properties, toxicity studies, and polymer applications for mainly controlled bioactive agents. As it has a rapid degradation, its main use was limited to the short-term controlled delivery of bioactive agents [157]. This controlled delivery has been studied using drugs for the treatment of several diseases, such as eye disorders, local anaesthetics, anticoagulants, and carcinogens [158-160].

Advanced delivery applications with adjustable release profiles require a certain type of product that can be obtained through microencapsulation, which are dynamic microcapsules. These can be carried out with a nondestructive trigger response mechanism, changing the permeability of membrane, which can be seen in hydrogels. Werner et al. reported an approach in obtaining reversibly responsive hydrogel microcapsules using reactive anhydride chemistry [161]. Through microfluidic double emulsion droplet models, poly(methacrylic anhydride) microcapsules are obtained, allowing the direct encapsulation of hydrophilic charge suspended in water inside the aqueous nucleus. The permeability of microcapsules is dependent on the degree of swelling and can be activated and modified. This fact enables the repeated capture and release of aqueous charge over several cycles. 


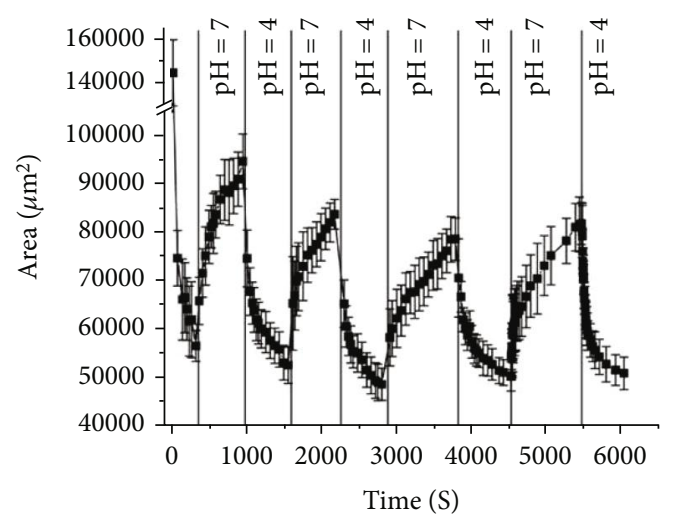

(a)
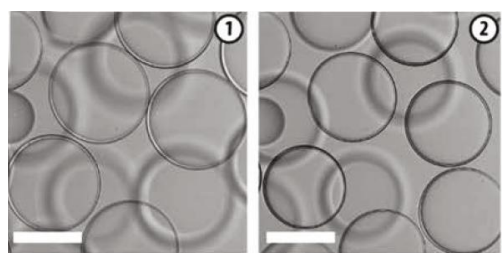

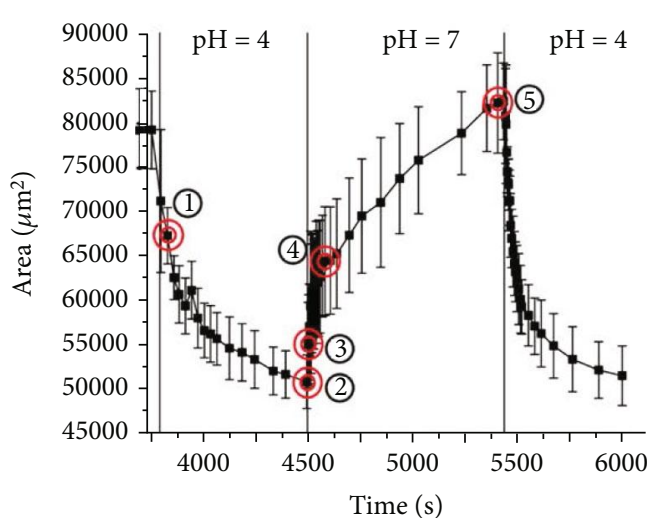

(b)
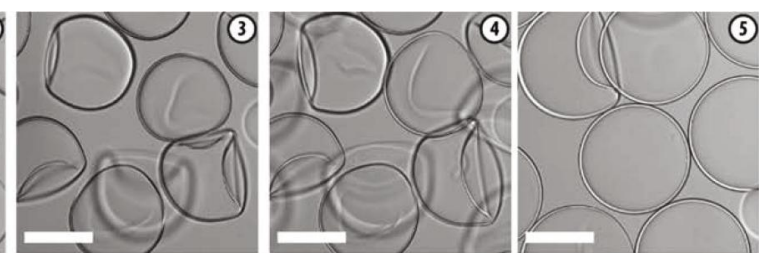

(c)

FIGURE 60: $(\mathrm{a}, \mathrm{b})$ Time-resolved size distribution (projected area) of the cyclic swelling $(\mathrm{pH}=7)$ and deswelling $(\mathrm{pH}=4)$ of poly $(\mathrm{methacrylic}$ anhydride-co-ethylene glycol dimethacrylate) (P(MAA-EGDMA)) hydrogel microcapsules with 98 mol \% acid content. Droplines represent time of $\mathrm{pH}$ change. (c) Selected bright-field microscopy images of the P(MAA - EGMDA) microcapsules during swelling and deswelling at the indicated time points in plot (b). All scale bars are $200 \mu \mathrm{m}$ [161].

Through several studies, the authors found that the change in $\mathrm{pH}$, size, and permeability of hydrogel microcapsules is repeatable, and these can be changed between their swollen and not swollen states. Regarding the size of microcapsules, dynamic properties were investigated. As shown in Figures 60(a) and 60(b), the swelling and disintegration of $\mathrm{pH}$-responsive microcapsules are quick and reversible. Capsule size varies after each $\mathrm{pH}$ change over the course of minutes and projected area of microcapsule is not indicative of the size of shell after the $\mathrm{pH}=7$. This is due to a change in shape during the swelling process: with an increase in $\mathrm{pH}$ from 4 to 7 , the shells swell in the plane, leading to an increase in the surface area of microcapsules. After each trigger event, microcapsules cannot fully balance their size, causing the microcapsule's responses to depend on their swelling history, which is not expected to be the same in each cycle. The microcapsules become spherical again after 15 minutes, due to the filling of nucleus with sufficient volume of water (Figure 60(c)).

(6) Poly(vinyl alcohol). Poly(vinyl alcohol) (Figure 61, PVA) is a water-soluble synthetic polymer used in the manufacture of paper, textiles, and in a variety of coatings. PVA is colourless (white) and odourless, being easily biodegradable [162].

PVA biodegradation has been studied in terms of microbial degradation and enzymatic degradation by secondary peroxidases (Figure 62) [163-165]. This biodegradation involves the enzymatic oxidation secondary alcohol groups. Other bacterial strains, such as Flavobacterium [165] and Acinetiobacter [166], were also effective in PVA degradation.<smiles>CC(O)CC(C)(C)C</smiles>

FIgURe 61: Chemical structure of PVA.

PVA is used as a polymer carrier for pesticides and herbicides $[167,168]$, and when used in low molecular weight form, it can be eliminated from organisms by glomerular filtration.

Regarding PVA microencapsulation, several studies have been reported. Galbiati et al. produced PVA microcapsules decorated with chitosan and loaded with a quinoline alkaloid that inhibits the DNA topoisomerase I pathway [169]. The objective of this research was to show the potential of microcapsules with PVA shell or microbubbles as injectable microdevices that support a theragnostic approach to different types of tumours.

Another recent study involves the integration of PVA microcapsules in porous collagen matrices to prepare new materials for dermatological or cosmetic applications [170]. Encapsulation technology has been used to improve the long-term stability of active substances and to increase and prolong effectiveness of active ingredients.

Figure 63 shows SEM micrographs of collagen matrices of PVA microcapsules with the addition of different amounts of retinyl palmitate in their core. It can be seen that the prepared matrices have a porous structure and spherical shape and a diameter between 20 and $60 \mu \mathrm{m}$. 


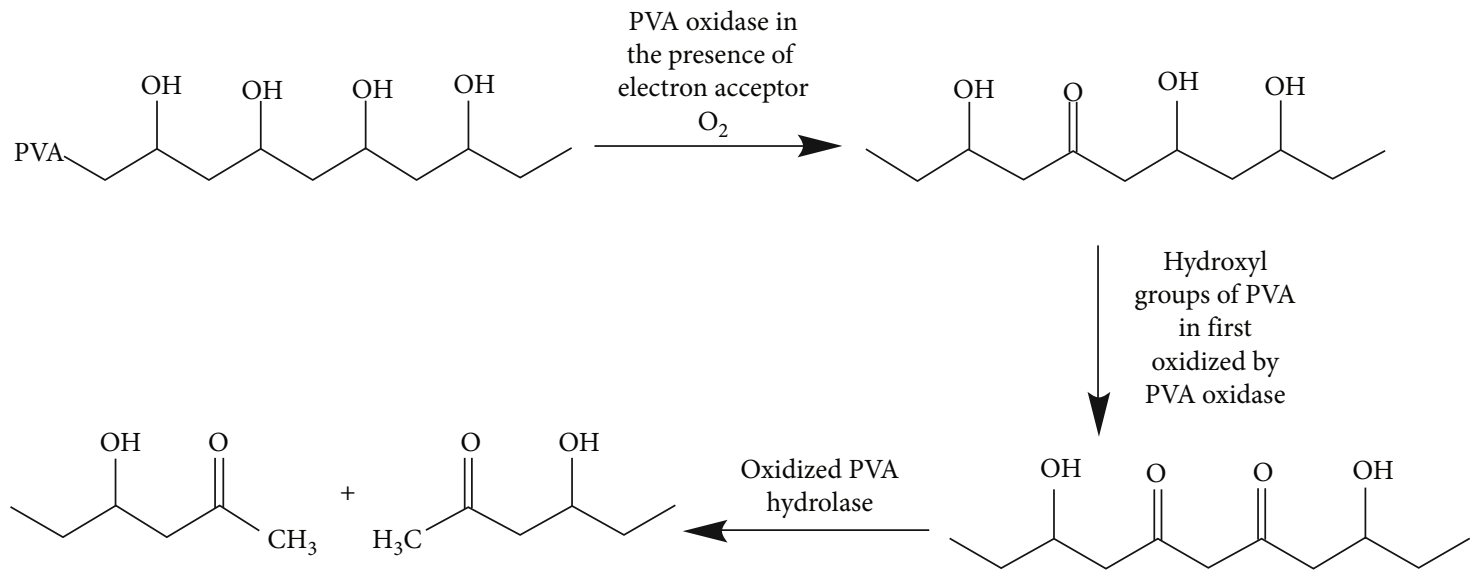

Figure 62: Possible pathway of PVA degradation.

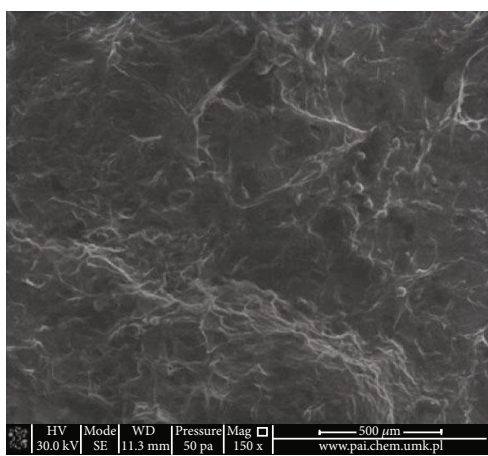

(a)

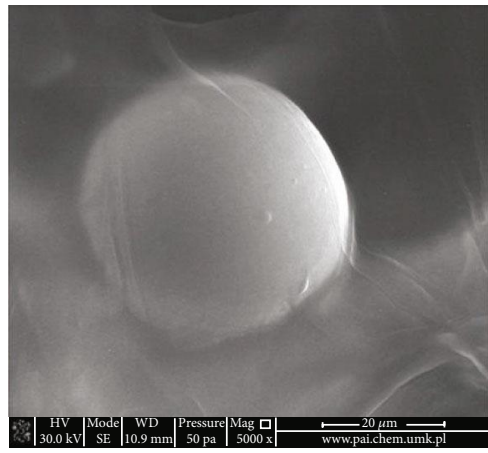

(c)

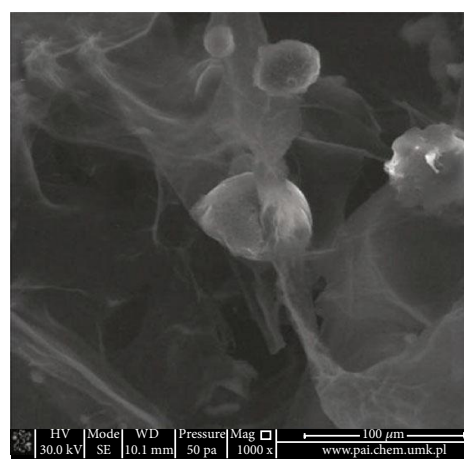

(e)

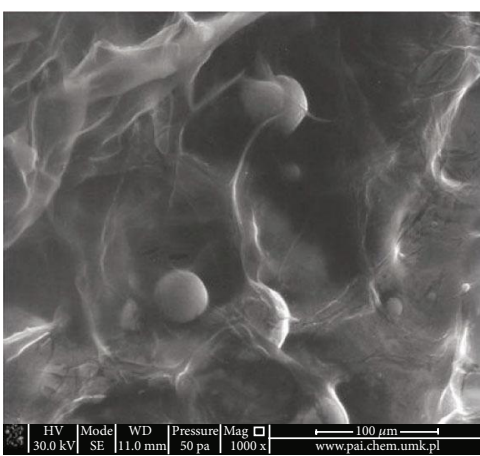

(b)

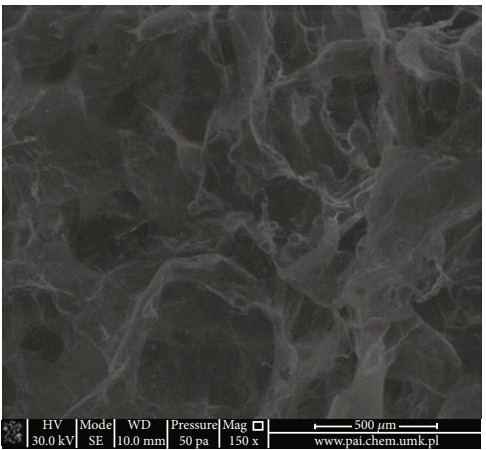

(d)

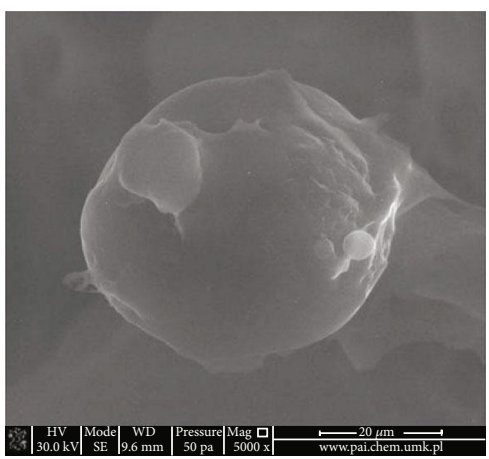

(f)

FIGURE 63: SEM micrographs of collagen matrix containing poly(vinyl) alcohol microcapsules loaded with retinyl palmitate: (a)-(c) Col/ PVA $1: 1$; (d)-(f) Col/PVA $1: 10$ [170]. 
Piacentini et al. developed poly(vinyl alcohol) (PVA) microparticles for the encapsulation of drugs with target properties (catechol (CA) and sodium diclofenac (DS)) [171]. The synthesis was carried out through membrane emulsification and chemical cross-linking. Several parameters were evaluated, such as the influence of emulsification method (membrane approach and homogenization), phase compositions (PVA concentration, drug concentration, physical, and chemical properties of the drug), crosslinking reaction conditions (concentration of cross-linking agent, acidic medium), the potential of microcapsules as carriers of drug release, and cytotoxicity in vitro.

Catechol encapsulation efficiency was close to $90 \%$ and was independent of the emulsification method, concentration of PVA, catechol, and PVA: glutaraldehyde (GA) ratio. On the other hand, DS encapsulation efficiency was higher when membrane emulsification was performed than homogenization, and it was higher when the PVA:GA ratio was increased.

The release of catechol was strictly dependent on the concentration of PVA (Figure 64). When the polymer concentration is 3.5 and $7 \%$, the release is similar with an amount of $47 \%$ catechol released after 4 hours. For the same release time, the amount of catechol released, at a PVA concentration of $15 \%$, is $60 \%$. The release of sodium diclofenac was negligible due to its conversion from salt to acid after the addition of benzoyl chloride.

The method proposed in this study allows the manufacture of hydrophilic polymeric particles with properties suitable for encapsulation of drugs. These target properties include particle size, particle size distribution, morphology/ structure and surface, encapsulation efficiency, and functional activity. This work is expected to provide information and increase the usefulness of hydrogels such as PVA for the development of drug delivery devices.

(7) Polyacrylates. Polyacrylates (Figure 65) are an important class of soft, resistant, and rubbery polymers, having a glass transition temperature below room temperature. They have a high transparency, good toughness, and impact elasticity and have good heat resistance.

Several polyacrylate derivatives are generally resistant to biodegradation [172]. Some studies on weight loss have been reported in soil burial tests for ethylene and propylene copolymers [173]. Poly(alkyl 2-cyanoacrylate) systems are polymerizable and adhere to wet surfaces and used in biomedical applications [174-176]. A polyacrylate derivative that has been widely used in biomedical areas due to its good biocompatibility is poly (2-hydroxyethyl methacrylate) $[177,178]$. An androgen has been covalently linked to a methacrylic and acrylic acid copolymer $[179,180]$, since there is a need for a spacer molecule between a drug and the carrier polymer in order to obtain an effective cleavage.

Sodium polyacrylate alginate microcapsules have been reported to encapsulate Lactobacillus plantarum MA2, and their feasibility and in vitro and in vivo release have been studied [181]. The seminatural edible polymer was

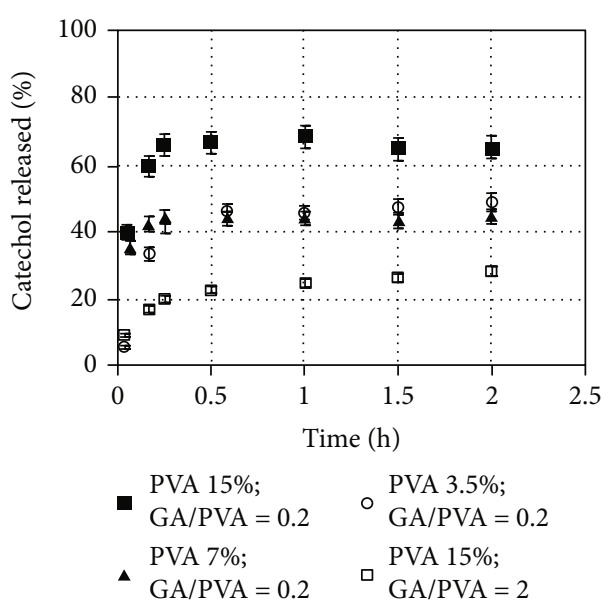

FIGURE 64: Catechol release as a function of time from PVA particles produced by membrane emulsification (ME), with different PVA concentration (3.5, 7, and $15 \mathrm{wt} . \%)$ and GA:PVA ratio (0.2 and 2) [171].<smiles>[R]OC(=O)C(CC(C)(C)[18F])C(C)(C)C</smiles>

Figure 65: Chemical structure of polyacrylate.

synthesized by grafting alginate sodium polyacrylate (PAAs) to improve the acid-resistant property of grafted Alg (Alg-PAAs (1: 2)).

According to the characterization, Liu et al. used several methods, one of which is the controlled release of microcapsules because the viable probiotics need to be released from them. It was concluded that the probiotic release percentage was negligible when exposed to stimulated gastric fluid (SGF) for 2 hours, while the microcapsules swelled a lot and became breakable in stimulated intestinal fluid (SIF) (Figure 66). Regarding the percentage of MA2 released in Alg-PAAs microcapsule $(1: 2)$, the release was significantly higher than in Alg microcapsule (58.9\% vs. $43.7 \%$ in 5 hours).

One aspect that contributed to minimize the harmful effect on the intestinal tract was the disintegration time of Alg-PAAs microcapsule $(1: 2)$, which was much shorter (6 hours) than that of Alg microcapsule (8 hours). Making a comparison with the Alg and Alg-PAAs microcapsules $(1: 2)$, it was found that the viability of MA2 in Alg-PAAs microcapsule $(1: 2)$ was increased, which may have been due to the better effect protector in SGF and the faster disintegration of Alg-PAA microcapsule $(1: 2)$ in SIF and stimulated colonic fluid (SCF).

In conclusion, Alg-PAAs microcapsule $(1: 2)$ can deliver probiotics more effectively and faster to the intestinal tract (including the large intestine) than Alg microcapsule.

Zhang et al. developed a flower garland fragrance encapsulation system using polyacrylate/paraffin microcapsules, 


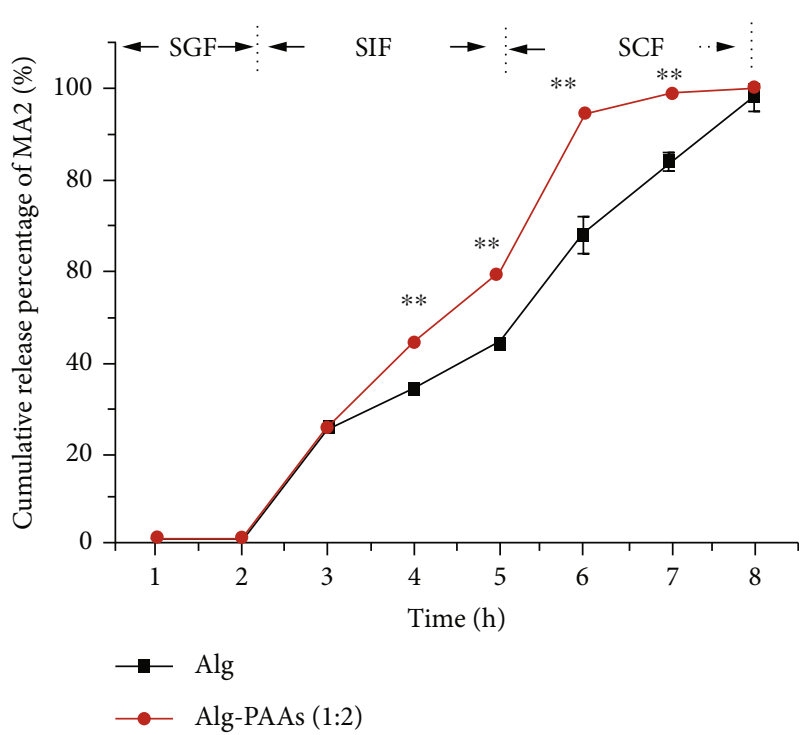

(a)

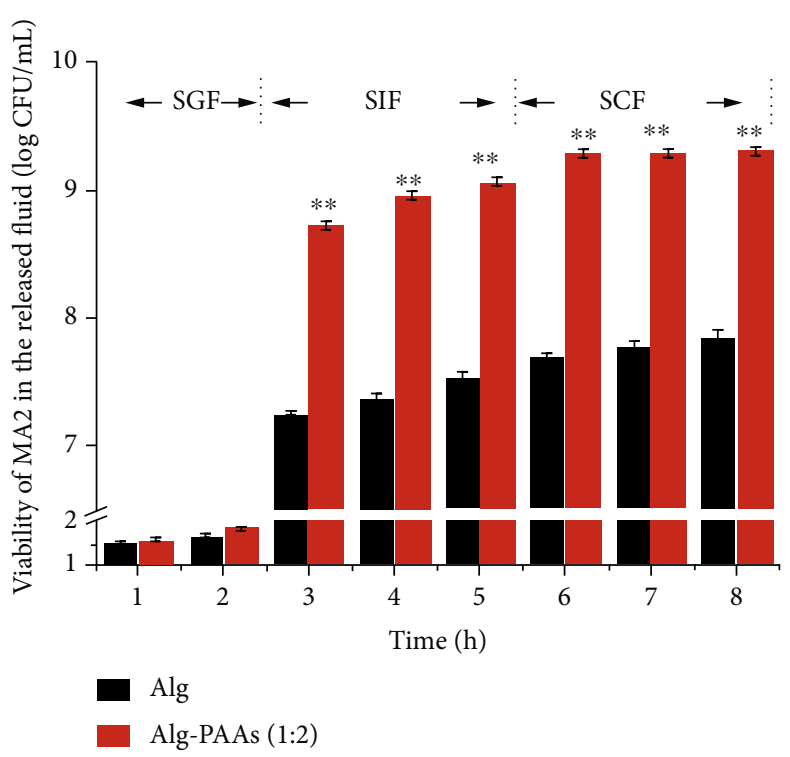

(b)

Figure 66: The release of L. plantarum MA2 in stimulated fluid. Alg or Alg-PAAs $(1: 2)$ microcapsules was immersed in SGF (pH 2.0, 2 hours), SIF ( $\mathrm{pH} 6.8,3$ hours), and SCF ( $\mathrm{pH} 7.4,3$ hours), consecutively. (a) Cumulative release percentage of L. plantarum MA2. (b) Viability of L. plantarum MA2 in the released fluid. Data were present as means $\pm \mathrm{SD}, n=3,{ }^{* *} p<0.01$ [181].

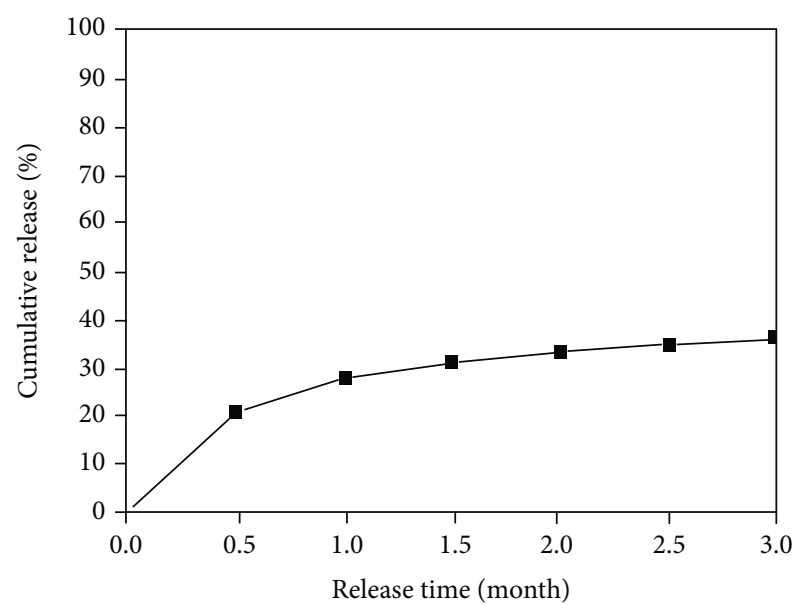

FIGURE 67: Release of flower garland fragrance from polyacrylate/ paraffin microcapsules in air [182].

obtained through the suspension polymerization method in Pickering emulsion [182]. The flower garland fragrance release was measured by using an infrared spectrophotometer. The results indicate that crosslinked PMMA/paraffin microcapsules and P(MMA-co-BMA)/paraffin microcapsules, prepared under ideal conditions, present a regular spherical shape and similar size distribution. Also, microcapsules of $\mathrm{P}(\mathrm{MMA}-\mathrm{co}-\mathrm{BMA}) /$ paraffin exhibit better thermal stability, with a temperature of thermal resistance of up to $184^{\circ} \mathrm{C}$.

The results also show that $63.9 \%$ of fragrance was retained after exposure of fragrance microcapsules to air for 3 months (Figure 67), and the fragrance continued to be released for more than 96 hours in surfactant solution (sodium lauryl sulfonate, $20 \mathrm{wt} \%$, Figure 68 ).

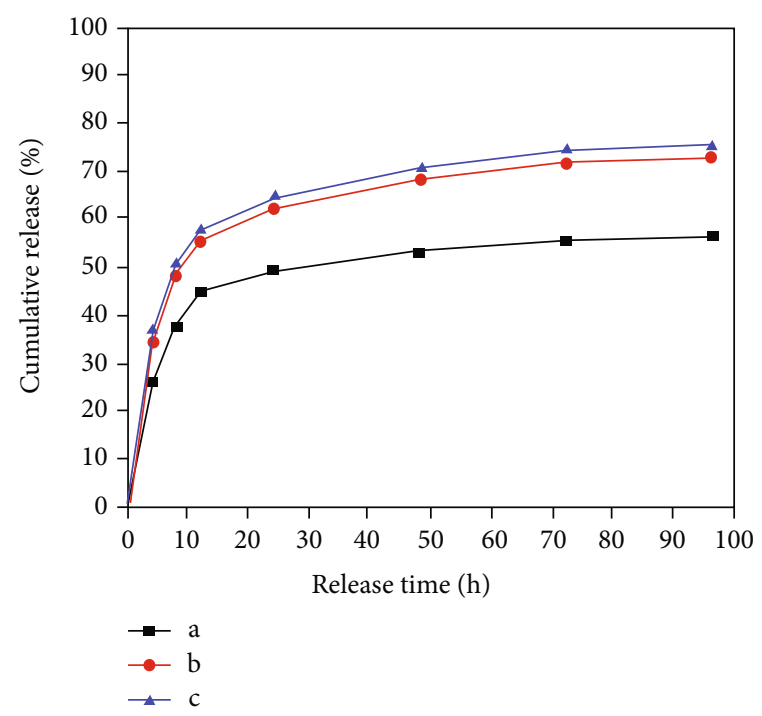

FIGURE 68: Release of flower garland fragrance from polyacrylate/ paraffin microcapsules in surfactant solution (SDS, $20 \mathrm{wt} \%$ ) under different storage temperatures: (a) $30^{\circ} \mathrm{C}$; (b) $40^{\circ} \mathrm{C}$; (c) $50^{\circ} \mathrm{C}$ [182].

The authors of this review consider that the results of fragrance release study are very promising, given that the microencapsulation system retains fragrance for 3 months when exposed to air. In addition, a challenging job was developed in the process of forming paraffin/polyacrylate microcapsules, with a high optimization of parameters such as the amount of cross-linking agent, water mass concentration, and type of comonomer, which influenced the properties of microcapsules. 


\section{Conclusion}

An in-depth review of different biodegradable polymers with promising results in microencapsulation systems is presented. The origin, main characteristics and applications, biodegradability, and an overview of most relevant scientific works with each polymer are some of the topics described in this article.

Lately, there is an increasing interest in the use of biodegradable polymers for microencapsulation systems, in particular due to the microplastics impact in the contamination of water resources. Microencapsulation technique is not new, having been used for several decades in different sectors. Over the years, different microencapsulation methods have been developed for distinct applications, such as controlled drug delivery, cosmetics, and textile industry. However, it is a research area that innovated quickly due to its promising applications and technological advances, which allowed the evolution and development of microencapsulation technologies.

Biodegradable polymers are very suitable for the development of new microencapsulation systems with merits of biodegradability and biocompatibility. It can be easily excreted out of body or into nature due to the ability to be eroded in small residues, which is not the case with nondegradable polymers. What makes these polymers good candidates are their mechanical properties, biocompatibility, and minimal cytotoxicity. However, there is still space to explore biodegradable polymers, and it takes time to manipulate the degradation pattern of polymers so that they do not produce any toxic products.

From the facts mentioned above, it is possible to conclude that synthesis of microcapsules with the use of biodegradable polymers has been an explored topic and in constant innovation. However, several developments can still be made in new applications, microencapsulation technologies, and new and optimized microencapsulation systems.

\section{Data Availability}

The raw/processed data required to reproduce these findings cannot be shared at this time due to technical or time limitations.

\section{Conflicts of Interest}

The authors declare that they have no conflicts of interest.

\section{Acknowledgments}

Joana F. Parente is grateful to the Project ReleaseME POCI-01-0247-FEDER-033268 and Vânia I. Sousa is grateful to the Project 4NoPressure - POCI-01-0247-FEDER039869, for their research grants from the Agência Nacional de Inovação, cofunded by the European Regional Development Fund (ERDF), through the Operational Programme for Competitiveness and Internationalisation (COMPETE 2020), under the PORTUGAL 2020 Partnership Agreement.
Juliana F. Marques and Marta A. Forte are grateful to the Fundação para a Ciência e Tecnologia (FCT) of Portugal, for their Ph.D grants, SFRH/BD/112868/2015 and PD/BD/ $128491 / 2017$, respectively. The authors also acknowledge the funding from FCT/PIDDAC through the Strategic Funds project reference UIDB/04650/2020-2023.

\section{References}

[1] F. Asghari, M. Samiei, K. Adibkia, A. Akbarzadeh, and S. Davaran, "Biodegradable and biocompatible polymers for tissue engineering application: a review," Artificial Cells, Nanomedicine, and Biotechnology, vol. 45, no. 2, pp. 185192, 2017.

[2] P. Kesharwani, K. Jain, and N. K. Jain, "Dendrimer as nanocarrier for drug delivery," Progress in Polymer Science, vol. 39, no. 2, pp. 268-307, 2014.

[3] S. Doppalapudi, A. Jain, W. Khan, and A. J. Domb, "Biodegradable polymers-an overview," Polymers for Advanced Technologies, vol. 25, no. 5, pp. 427-435, 2014.

[4] I. Armentano, D. Puglia, F. Luzi et al., "Nanocomposites based on biodegradable polymers," Materials (Basel), vol. 11, no. 5, p. 795, 2018.

[5] A. C. Hunter and S. M. Moghimi, "Smart polymers in drug delivery: a biological perspective," Polymer Chemistry, vol. 8, no. 1, pp. 41-51, 2017.

[6] M. Fathi and J. Barar, "Perspective highlights on biodegradable polymeric nanosystems for targeted therapy of solid tumors," Bio Impacts, vol. 7, no. 1, pp. 49-57, 2017.

[7] S. K. Ghosh, Functional coatings and microencapsulation: a general perspective, Functional Coatings: by Polymer Microencapsulation, 2006.

[8] I. M. Martins, M. F. Barreiro, M. Coelho, and A. E. Rodrigues, "Microencapsulation of essential oils with biodegradable polymeric carriers for cosmetic applications," Chemical Engineering Journal, vol. 245, pp. 191-200, 2014.

[9] G. Nelson, Microencapsulated colourants for technical textile application, Woodhead Publishing Series in Textiles, 2013.

[10] A. C. G. Moreira, Microencapsulação de Óleos Essenciais, pp. $1-48,2014$.

[11] S. K. Prajapati, A. Jain, A. Jain, and S. Jain, "Biodegradable polymers and constructs: a novel approach in drug delivery," European Polymer Journal, vol. 120, article 109191, 2019.

[12] S. Bhatia, Natural polymer drug delivery systems: Nanoparticles, plants, and algae, Springer, 2016.

[13] M. S. Huh, E. J. Lee, H. Koo et al., Polysaccharide-based nanoparticles for gene delivery, Springer, 2017.

[14] A. Jain, A. Gulbake, S. Shilpi, A. Jain, P. Hurkat, and S. K. Jain, "A new horizon in modifications of chitosan: syntheses and applications," Critical Reviews in Therapeutic Drug Carrier Systems, vol. 30, no. 2, pp. 91-181, 2013.

[15] J. H. Cummings, S. Milojevic, M. Harding et al., "In vivo studies of amylose- and ethylcellulose-coated $\left[{ }^{13} \mathrm{C}\right]$ glucose microspheres as a model for drug delivery to the colon," Journal of Controlled Release, vol. 40, no. 1-2, pp. 123-131, 1996.

[16] Y. Changhong, L. Xiongwei, C. Xiaoli et al., "Anticancer gelatin microspheres with multiple functions," Biomaterials, vol. 12, no. 7, pp. 640-644, 1991.

[17] A. C. Hotel and A. Cordoba, "Health and nutritional properties of probiotics in food including powder milk with live lactic acid bacteria," Prevention, vol. 5, pp. 1-10, 2001. 
[18] A. Homayouni, A. Azizi, M. R. Ehsani, M. S. Yarmand, and S. H. Razavi, "Effect of microencapsulation and resistant starch on the probiotic survival and sensory properties of synbiotic ice cream," Food Chemistry, vol. 111, no. 1, pp. 50-55, 2008.

[19] C. L. Vernazza, B. A. Rabiu, and G. R. Gibson, "Human colonic microbiology and the role of dietary intervention: introduction to prebiotics," in Prebiotics: Development and application, pp. 1-28, John Wiley and Sons, Ltd, 2012.

[20] W. S. Cheow, T. Y. Kiew, and K. Hadinoto, "Effects of adding resistant and waxy starches on cell density and survival of encapsulated biofilm of Lactobacillus rhamnosus GG probiotics," Science and Technology, vol. 69, pp. 497-505, 2016.

[21] M. R. Corbo, A. Bevilacqua, M. Gallo, B. Speranza, and M. Sinigaglia, "Immobilization and microencapsulation of Lactobacillus plantarum: performances and in vivo applications," Innovative Food Science and Emerging Technologies, vol. 18, pp. 196-201, 2013.

[22] Z. Muhammad, R. Ramzan, G. C. Huo, H. Tian, and X. Bian, "Integration of polysaccharide-thermoprotectant formulations for microencapsulation of Lactobacillus plantarum, appraisal of survivability and physico-biochemical properties during storage of spray dried powders," Food Hydrocolloids, vol. 66, pp. 286-295, 2017.

[23] M. de Araújo Etchepare, G. C. Raddatz, A. J. Cichoski et al., "Effect of resistant starch (Hi-maize) on the survival of Lactobacillus acidophilus microencapsulated with sodium alginate," Journal of Functional Foods, vol. 21, pp. 321-329, 2016.

[24] D. Y. Ying, S. Schwander, R. Weerakkody, L. Sanguansri, C. Gantenbein-Demarchi, and M. A. Augustin, "Microencapsulated Lactobacillus rhamnosus GG in whey protein and resistant starch matrices: probiotic survival in fruit juice," Journal of Functional Foods, vol. 5, no. 1, pp. 98-105, 2013.

[25] V. D. Vilivalam, L. Illum, and K. Iqbal, "Starch capsules: an alternative system for oral drug delivery," Pharmaceutical Science \& Technology Today, vol. 3, no. 2, pp. 64-69, 2000.

[26] R. Pérez-Masiá, R. López-Nicolás, M. J. Periago, G. Ros, J. M. Lagaron, and A. López-Rubio, "Encapsulation of folic acid in food hydrocolloids through nanospray drying and electrospraying for nutraceutical applications," Food Chemistry, vol. 168, pp. 124-133, 2015.

[27] D. Li, B. Liu, F. Yang, X. Wang, H. Shen, and D. Wu, "Preparation of uniform starch microcapsules by premix membrane emulsion for controlled release of avermectin," Carbohydrate Polymers, vol. 136, pp. 341-349, 2016.

[28] X. Cai, X. Du, D. Cui, X. Wang, Z. Yang, and G. Zhu, "Improvement of stability of blueberry anthocyanins by carboxymethyl starch/xanthan gum combinations microencapsulation," Food Hydrocolloids, vol. 91, pp. 238-245, 2019.

[29] W. Tangsongcharoen, P. Punyamoonwongsa, and P. Chaiyasat, "High performance biocompatible cellulosebased microcapsules encapsulating gallic acid prepared by inverse microsuspension polymerization," Polymer International, vol. 68, no. 4, pp. 714-723, 2019.

[30] K. Chen, C. Xu, J. Zhou, R. Zhao, Q. Gao, and C. Wang, "Multifunctional fabric coatings with slow-releasing fragrance and UV resistant properties from ethyl cellulose/silica hybrid microcapsules," Carbohydrate Polymers, vol. 232, article 115821, 2020.

[31] M. G. Simões, P. Coimbra, A. S. Carreira, M. M. Figueiredo, M. H. Gil, and P. N. Simões, "Eugenol-loaded microspheres incorporated into textile substrates," Cellulose, vol. 27, no. 7, pp. 4109-4121, 2020.

[32] J. Hosokawa, M. Nishiyama, K. Yoshihara, and T. Kubo, "Biodegradable film derived from chitosan and homogenized cellulose," Industrial and Engineering Chemistry Research, vol. 29, no. 5, pp. 800-805, 1990.

[33] I. Tokura, S. Miura, Y. Uraki, Y. Watanabe, K. Saiki, and I. Azuma, "Biodegradable chitin derivative as various types of drug carriers," in Polymer Chemistry, Am Chem Soc Polym Prep. Div, 1990.

[34] M. Tachibana, A. Yaita, H. Taniura, K. Fukasawa, N. Nagasue, and T. Nakamura, "The use of chitin as a new absorbable suture material an experimental study," The Japanese Journal of Surgery, vol. 18, no. 5, pp. 533-539, 1988.

[35] J. Peña, I. Izquierdo-Barba, A. Martínez, and M. Vallet-Regí, "New method to obtain chitosan/apatite materials at room temperature," Solid State Sciences, vol. 8, no. 5, pp. 513-519, 2006.

[36] A. K. Singla and M. Chawla, "Chitosan: some pharmaceutical and biological aspects - an update," The Journal of Pharmacy and Pharmacology, vol. 53, pp. 1047-1067, 2010.

[37] K. De Yao, Y. J. Yin, M. X. Xu, and Y. F. Wang, "Investigation of $\mathrm{pH}$-sensitive drug delivery system of chitosan/gelatin hybrid polymer network," Polymer International, vol. 38, no. 1, pp. 77-82, 1995.

[38] F. Chellat, M. Tabrizian, S. Dumitriu, E. Chornet, C. H. Rivard, and L. Yahia, "Study of biodegradation behavior of chitosan-xanthan microspheres in simulated physiological media," Journal of Biomedical Materials Research, vol. 53, no. 5, pp. 592-599, 2000.

[39] M. A. Bayoni, "Influence of polymers weight ratio and $\mathrm{pH}$ of polymers solution on the characteristics of chitosan carboxymethyl cellulose microspheres containig theophyline," Bollettino Chimico Farmaceutico, vol. 142, pp. 336-342, 2003.

[40] T. Yamaguchi, Y. Ito, and N. Shibuya, "Oligosaccharide elicitors and their receptors for plant defense responses," Trends in Glycoscience and Glycotechnology, vol. 12, no. 64, pp. 113120, 2000.

[41] K. Struszczyk and H. Pospieszny, "Kotlinski, Some new applications of chitosan in agriculture," in Chitin and Chitosan Sources, Chemistry, Biochemistry, Physical Properties and Applications, G. S. Bræk, T. Anthonsen, and P. Sandford, Eds., pp. 733-742, Elsevier Appl. Sci., London, 1989.

[42] G. W. Gooday, "The Ecology of Chitin Degradation," in Advances in microbial ecology, pp. 387-430, Springer, Boston, MA, 1990.

[43] G. Galed, M. E. Fernández-Valle, A. Martínez, and A. Heras, "Application of MRI to monitor the process of ripening and decay in citrus treated with chitosan solutions," Magnetic Resonance Imaging, vol. 22, no. 1, pp. 127-137, 2004.

[44] W. C. Hsieh, C. P. Chang, and Y. L. Gao, "Controlled release properties of chitosan encapsulated volatile citronella oil microcapsules by thermal treatments," Colloids and Surfaces. B, Biointerfaces, vol. 53, no. 2, pp. 209-214, 2006.

[45] X. T. Mu, Y. Li, X. J. Ju et al., "Microfluidic fabrication of structure-controlled chitosan microcapsules via interfacial cross-linking of droplet templates," ACS Applied Materials \& Interfaces, vol. 12, no. 51, pp. 57514-57525, 2020.

[46] A. A. Badwan, A. Abumalooh, E. Sallam, A. Abukalaf, and O. Jawan, “A sustained release drug delivery system using 
calcium alginate beads," Drug Development and Industrial Pharmacy, vol. 11, no. 2-3, pp. 239-256, 1985.

[47] T. Yotsuyanagi, T. Ohkubo, T. Ohhashi, and K. Ikeda, "Calcium-induced gelation of alginic acid and $\mathrm{pH}$-sensitive reswelling of dried gels," Chemical \& Pharmaceutical Bulletin, vol. 35, no. 4, pp. 1555-1563, 1987.

[48] G. Pfister, M. Bahadir, and F. Korte, "Release characteristics of herbicides from $\mathrm{Ca}$ alginate gel formulations," Journal of Controlled Release, vol. 3, no. 1-4, pp. 229-233, 1986.

[49] E. G. Posillico, "Microencapsulation technology for largescale antibody production," Biotechnology, vol. 4, p. 114, 1986.

[50] G. A. King, A. J. Daugulis, P. Faulkner, and M. F. A. Goosen, "Alginate-polylysine microcapsules of controlled membrane molecular weight cutoff for mammalian cell culture engineering," Biotechnology Progress, vol. 3, no. 4, pp. 231-240, 1987.

[51] K. K. Kwok, M. J. Groves, and D. J. Burgess, "Production of 5$15 \mu \mathrm{m}$ diameter alginate-polylysine microcapsules by an airatomization technique," Pharmaceutical Research, vol. 8, no. 3, pp. 341-344, 1991.

[52] S. Wittaya-Areekul, J. Kruenate, and C. Prahsarn, "Preparation and in vitro evaluation of mucoadhesive properties of alginate/chitosan microparticles containing prednisolone," International Journal of Pharmaceutics, vol. 312, no. 1-2, pp. 113-118, 2006.

[53] S. Jurić, E. Đermić, S. Topolovec-Pintarić, M. Bedek, and M. Vinceković, "Physicochemical properties and release characteristics of calcium alginate microspheres loaded with Trichoderma viride spores," Journal of Integrative Agriculture, vol. 18, no. 11, pp. 2534-2548, 2019.

[54] N. T. T. Uyen, Z. A. A. Hamid, and A. Nurazreena, "Fabrication and characterization of alginate microspheres," Materials Today: Proceedings, vol. 17, pp. 792-797, 2019.

[55] A. Faidi, M. A. Lassoued, M. E. H. Becheikh, M. Touati, J. F. Stumbé, and F. Farhat, "Application of sodium alginate extracted from a Tunisian brown algae Padina pavonica for essential oil encapsulation: microspheres preparation, characterization and in vitro release study," International Journal of Biological Macromolecules, vol. 136, pp. 386-394, 2019.

[56] D. Dhamecha, R. Movsas, U. Sano, and J. U. Menon, “Applications of alginate microspheres in therapeutics delivery and cell culture: past, present and future," International Journal of Pharmaceutics, vol. 569, article 118627, 2019.

[57] L. Agüero, D. Zaldivar-Silva, L. Peña, and M. Dias, "Alginate microparticles as oral colon drug delivery device: a review," Carbohydrate Polymers, vol. 168, pp. 32-43, 2017.

[58] G. Klöck, H. Frank, R. Houben et al., "Production of purified alginates suitable for use in immunoisolated transplantation," Applied Microbiology and Biotechnology, vol. 40, no. 5, pp. 638-643, 1994.

[59] P. De Vos, B. J. De Haan, G. H. J. Wolters, J. H. Strubbe, and R. Van Schilfgaarde, "Improved biocompatibility but limited graft survival after purification of alginate for microencapsulation of pancreatic islets," Diabetologia, vol. 40, no. 3, pp. 262-270, 1997.

[60] E. A. Nunamaker, E. K. Purcell, and D. R. Kipke, "In vivo stability and biocompatibility of implanted calcium alginate disks," Journal of Biomedical Materials Research, vol. 83A, no. 4, pp. 1128-1137, 2007.

[61] U. Zimmermann, K. Federlin, K. Hannig et al., "Production of mitogen-contamination free alginates with variable ratios of mannuronic acid to guluronic acid by free flow electrophoresis," Methods, vol. 13, no. 1, pp. 269-274, 1992.

[62] S. K. Tam, J. Dusseault, S. Bilodeau, G. Langlois, J. P. Hallé, and L. Yahia, "Factors influencing alginate gel biocompatibility," Journal of Biomedical Materials Research Part A, vol. 98A, no. 1, pp. 40-52, 2011.

[63] H. K. Holme, K. Lindmo, A. Kristiansen, and O. Smidsrød, "Thermal depolymerization of alginate in the solid state," Carbohydrate Polymers, vol. 54, no. 4, pp. 431-438, 2003.

[64] P. Gacesa, "Enzymic degradation of alginates," The International Journal of Biochemistry, vol. 24, no. 4, pp. 545-552, 1992.

[65] K. Y. Lee and D. J. Mooney, "Alginate: properties and biomedical applications," Progress in Polymer Science, vol. 37, no. 1, pp. 106-126, 2012.

[66] G. Orive, E. Santos, D. Poncelet et al., "Cell encapsulation: technical and clinical advances," Trends in Pharmacological Sciences, vol. 36, no. 8, pp. 537-546, 2015.

[67] M. Zhao, F. Qu, Z. Wu, K. Nishinari, G. O. Phillips, and Y. Fang, "Protection mechanism of alginate microcapsules with different mechanical strength for Lactobacillus plantarum ST-III," Food Hydrocolloids, vol. 66, pp. 396-402, 2017.

[68] D. Diel, V. L. Lagranha, R. S. Schuh, F. Bruxel, U. Matte, and H. F. Teixeira, "Optimization of alginate microcapsules containing cells overexpressing $\alpha$-L-iduronidase using BoxBehnken design," European Journal of Pharmaceutical Sciences, vol. 111, pp. 29-37, 2018.

[69] X. Sun, R. G. Cameron, and J. Bai, "Microencapsulation and antimicrobial activity of carvacrol in a pectin-alginate matrix," Food Hydrocolloids, vol. 92, pp. 69-73, 2019.

[70] A. A. Eastoe and J. E. Leach, "The Science and Technology of Gelatin," in Sci. Technol. Gelatin, A. G. Ward and A. Courts, Eds., p. 73, Academic, New York, 1977.

[71] Y. Tabata and Y. Ikada, "Macrophage activation through phagocytosis of muramyl dipeptide encapsulated in gelatin microspheres," The Journal of Pharmacy and Pharmacology, vol. 39, pp. 698-704, 2011.

[72] Y. Tabata and Y. Ikada, "Synthesis of gelatin microspheres containing interferon," Pharmaceutical Research, vol. 6, no. 5, pp. 422-427, 1989.

[73] Y. Tabata, K. Uno, S. Muramatsu, and Y. Ikada, "In vivo effects of recombinant interferon alpha A/D incorporated in gelatin microspheres on murine tumor cell growth," Japanese Journal of Cancer Research, vol. 80, no. 4, pp. 387-393, 1989.

[74] Y. Tabata, K. Uno, Y. Ikada, and S. Muramatsu, "Suppressive effect of recombinant TNF-gelatin conjugate on murine tumour growth in-vivo," The Journal of Pharmacy and Pharmacology, vol. 45, no. 4, pp. 303-308, 1993.

[75] Y. Tabata, K. Uno, Y. Ikada, T. Kishida, and S. Muramatsu, "Potentiation ofin vivoAntitumor effects of recombinant interleukin- $1 \alpha$ by gelatin conjugation," Japanese Journal of Cancer Research, vol. 84, no. 6, pp. 681-688, 1993.

[76] T. Yasuhiko and I. Yoshito, “Targeting of muramyl dipeptide to macrophages by gelatin conjugation to enhance their in vivo antitumor activity," Journal of Controlled Release, vol. 27, no. 1, pp. 79-88, 1993.

[77] K. R. Kamath and K. Park, "Use of gamma irradiation for the preparation of hydrogels from natural polymers," in Proc. Int. Symp. Control. Release Bioact. Mater., vol. 19, pp. 42-43, Orlando, Florida, 1992. 
[78] G. R. Ziegler, "Microstructure of mixed gelatin-egg white gels: impact on rheology and application to microparticulation," Biotechnology Progress, vol. 7, no. 3, pp. 283-287, 1991.

[79] S. Shinde and B. G. Erhan, "Flexibilized gelatin film-based artificial skin model: II. Release kinetics of incorporated bioactive molecules," Bio-Medical Materials and Engineering, vol. 2, no. 3, pp. 127-131, 1992.

[80] B. G. Shinde, V. S. Nithianandam, K. Kaleem, and S. Erhan, "Flexibilized gelatin film-based artificial skin model: I. preparation and properties of the films," Bio-Medical Materials and Engineering, vol. 2, no. 3, pp. 123-126, 1992.

[81] K. A. Piez and J. Gross, "The amino acid composition of some fish collagens: the relation between composition and structure," The Journal of Biological Chemistry, vol. 235, no. 4, pp. 995-998, 1960.

[82] W. F. Harrington and N. V. Rao, "Pyrrolidine residues and the stability of collagen," in Conform. Biopolym., pp. 513531, Academic Press, 1967.

[83] B. H. Leuenberger, "Investigation of viscosity and gelation properties of different mammalian and fish gelatins," Topics in Catalysis, vol. 5, no. 4, pp. 353-361, 1991.

[84] A. I. Sarabia, M. C. Gómez-Guillén, and P. Montero, “The effect of added salts on the viscoelastic properties of fish skin gelatin," Food Chemistry, vol. 70, no. 1, pp. 71-76, 2000.

[85] I. J. Haug, K. I. Draget, and O. Smidsrød, "Physical and rheological properties of fish gelatin compared to mammalian gelatin," Food Hydrocolloids, vol. 18, no. 2, pp. 203-213, 2004.

[86] A. Gharsallaoui, G. Roudaut, O. Chambin, A. Voilley, and R. Saurel, "Applications of spray-drying in microencapsulation of food ingredients: an overview," Food Research International, vol. 40, no. 9, pp. 1107-1121, 2007.

[87] X. D. Liu, T. Atarashi, T. Furuta et al., "Microencapsulation of emulsified hydrophobic flavors by spray drying," Drying Technology, vol. 19, no. 7, pp. 1361-1374, 2001.

[88] B. Shu, W. Yu, Y. Zhao, and X. Liu, "Study on microencapsulation of lycopene by spray-drying," Journal of Food Engineering, vol. 76, no. 4, pp. 664-669, 2006.

[89] Ö. A. Bayram, M. Bayram, and A. R. Tekin, "Spray drying of sumac flavour using sodium chloride, sucrose, glucose and starch as carriers," Journal of Food Engineering, vol. 69, no. 2, pp. 253-260, 2005.

[90] A. M. Bakry, S. Abbas, B. Ali et al., "Microencapsulation of oils: a comprehensive review of benefits, techniques, and applications," Comprehensive Reviews in Food Science and Food Safety, vol. 15, no. 1, pp. 143-182, 2016.

[91] T. K. J. Bower, G. Chaundy, and F. K. Kilbride, "Process for incorporating a material in crosslinked gelatin, and product therform," 1992, US Patent 5-153177.

[92] S. Diguet, K. Feltes, N. Kleemann, B. Leuenberger, and J. Ulm, "Extrusion-stable poly-unsaturated fatty-acid compositions for food products," 2005, US Patent 13-113543.

[93] B. Leuenberger, J. C. Tritsch, and J. Ulm, "Process for preparing fat soluble beadlets," 2002, US Patent 6-444227.

[94] L. J. Scialpi, Process for Preparing Fat-Soluble Vitamin Active Beadlets, 1987, US Patent 4-670247.

[95] K. Iwanaga, T. Yabuta, M. Kakemi, K. Morimoto, Y. Tabata, and Y. Ikada, "Usefulness of microspheres composed of gelatin with various cross-linking density," Journal of Microencapsulation, vol. 20, no. 6, pp. 767-776, 2003.

[96] T. Morita, Y. Horikiri, T. Suzuki, and H. Yoshino, "Preparation of gelatin microparticles by co-lyophilization with poly (ethylene glycol): characterization and application to entrapment into biodegradable microspheres," International Journal of Pharmaceutics, vol. 219, no. 1-2, pp. 127-137, 2001.

[97] Y. Q. Kong, D. Li, L. J. Wang, and B. Adhikari, "Preparation of gelatin microparticles using water-in-water (w/w) emulsification technique," Journal of Food Engineering, vol. 103, no. 1, pp. 9-13, 2011.

[98] R. Dinarvand, S. Mahmoodi, E. Farboud, M. Salehi, and F. Atyabi, "Preparation of gelatin microspheres containing lactic acid-effect of cross-linking on drug release," Acta Pharmaceutica, vol. 55, no. 1, pp. 57-67, 2005.

[99] M. Yamamoto, Y. Ikada, and Y. Tabata, "Controlled release of growth factors based on biodegradation of gelatin hydrogel," Journal of Biomaterials Science Polymer Edition, vol. 12, no. 1, pp. 77-88, 2001.

[100] S. Magdassi and Y. Vinetsky, "Microencapsulation of o/w emulsions by formation of a protein-surfactant insoluble complex," Journal of Microencapsulation, vol. 12, no. 5, pp. 537-545, 1995.

[101] V. B. Junyaprasert, A. Mitrevej, N. Sinchaipanid, P. Boonme, and D. E. Wurster, "Effect of process variables on the microencapsulation of vitamin A palmitate by gelatin-acacia coacervation," Drug Development and Industrial Pharmacy, vol. 27, no. 6, pp. 561-566, 2001.

[102] D. F. Silva, C. S. Favaro-Trindade, G. A. Rocha, and M. Thomazini, "Microencapsulation of lycopene by gelatinpectin complex coacervation," Journal of Food Processing \& Preservation, vol. 36, no. 2, pp. 185-190, 2012.

[103] N. Yan, Encapsulation Agglomeration of Microcapsules and Method for the Preparation Thereof, 2010, United States patent US 8,968,872, 2015.

[104] K. Nakagawa and H. Nagao, "Microencapsulation of oil droplets using freezing-induced gelatin-acacia complex coacervation," Colloids and Surfaces A: Physicochemical and Engineering Aspects, vol. 411, pp. 129-139, 2012.

[105] A. Bhattacharyya and J. F. Argillier, "Microencapsulation by complex coacervation: effect of cationic surfactants," Journal of Surface Science and Technology, vol. 21, pp. 161-168, 2005.

[106] W. Li, G. Wu, H. Chen, and M. Wang, "Preparation and characterization of gelatin/SDS/NaCMC microcapsules with compact wall structure by complex coacervation," Colloids and Surfaces A: Physicochemical and Engineering Aspects, vol. 333, no. 1-3, pp. 133-137, 2009.

[107] S. T. Lim, G. P. Martin, D. J. Berry, and M. B. Brown, "Preparation and evaluation of the in vitro drug release properties and mucoadhesion of novel microspheres of hyaluronic acid and chitosan," Journal of Controlled Release, vol. 66, no. 2-3, pp. 281-292, 2000.

[108] Y. Yeo, E. Bellas, W. Firestone, R. Langer, and D. S. Kohane, "Complex coacervates for thermally sensitive controlled release of flavor compounds," Journal of Agricultural and Food Chemistry, vol. 53, no. 19, pp. 7518-7525, 2005.

[109] J. Liu, C. Liu, Y. Liu, M. Chen, Y. Hu, and Z. Yang, "Study on the grafting of chitosan-gelatin microcapsules onto cotton fabrics and its antibacterial effect," Colloids and Surfaces. B, Biointerfaces, vol. 109, pp. 103-108, 2013.

[110] J. Fraj, L. Petrović, L. Đekić, J. M. Budinčić, S. Bučko, and J. Katona, "Encapsulation and release of vitamin C in double $\mathrm{W} / \mathrm{O} / \mathrm{W}$ emulsions followed by complex coacervation in gelatin-sodium caseinate system," Journal of Food Engineering, vol. 292, article 110353, 2021. 
[111] J. S. F. de Araújo, E. L. de Souza, J. R. Oliveira et al., "Microencapsulation of sweet orange essential oil (Citrus aurantium var. dulcis) by liophylization using maltodextrin and maltodextrin/gelatin mixtures: preparation, characterization, antimicrobial and antioxidant activities," International Journal of Biological Macromolecules, vol. 143, pp. 991-999, 2020.

[112] A. B. Balaji, H. Pakalapati, M. Khalid, R. Walvekar, and $\mathrm{H}$. Siddiqui, Natural and synthetic biocompatible and biodegradable polymers, Elsevier Ltd, 2018.

[113] J. E. Potts, R. A. Clendinning, W. B. Ackart, and W. D. Niegisch, "The biodegradability of synthetic polymers," in Polymers and ecological problems, pp. 61-79, Springer, 1973.

[114] Y. Tokiwa and T. Suzuki, "Hydrolysis of copolyesters containing aromatic and aliphatic ester blocks by lipase," Journal of Applied Polymer Science, vol. 26, no. 2, pp. 441-448, 1981.

[115] X. Yu and J. Zhu, "A facile way to fabricate polyester microcapsules," Journal of Controlled Release, vol. 152, Supplement 1, pp. e128-e129, 2011.

[116] N. Q. Shi, J. Zhou, J. Walker et al., "Microencapsulation of luteinizing hormone-releasing hormone agonist in poly (lactic- co -glycolic acid) microspheres by spray-drying," Journal of Controlled Release, vol. 321, pp. 756-772, 2020.

[117] A. Middleton and J. Tipton, "Synthetic biodegradable polymers as medical devices," Medical Plastic and Biomaterials, vol. 5, pp. 30-39, 1998.

[118] W. C. Ko, C. K. Chang, H. J. Wang, S. J. Wang, and C. W. Hsieh, "Process optimization of microencapsulation of curcumin in $\gamma$-polyglutamic acid using response surface methodology," Food Chemistry, vol. 172, pp. 497-503, 2015.

[119] D. K. Gilding and A. M. Reed, "Biodegradable polymers for use in surgery-polyglycolic/poly (actic acid) homo- and copolymers: 1," Polymer, vol. 20, no. 12, pp. 1459-1464, 1979.

[120] R. K. Kulkarni, E. G. Moore, A. F. Hegyeli, and F. Leonard, "Biodegradable poly(lactic acid) polymers," Journal of Biomedical Materials Research, vol. 5, no. 3, pp. 169-181, 1971.

[121] E. J. Frazza and E. E. Schmitt, "A new absorbable suture," Journal of Biomedical Materials Research, vol. 5, no. 2, pp. 43-58, 1971.

[122] L. R. Beck, D. R. Cowsar, D. H. Lewis et al., "A new longacting injectable microcapsule system for the administration of progesterone," Fertility and Sterility, vol. 31, no. 5, pp. 545-551, 1979.

[123] K. Chronaki, D. M. Korres, C. D. Papaspyrides, and S. Vouyiouka, "Poly(lactic acid) microcapsules: tailoring properties via solid state polymerization," Polymer Degradation and Stability, vol. 179, article 109283, 2020.

[124] R. D. Fields, F. Rodriguez, and R. K. Finn, "Microbial degradation of polyesters: polycaprolactone degraded by P. pullulans," Journal of Applied Polymer Science, vol. 18, no. 12, pp. 3571-3579, 1974.

[125] C. V. Benedict, W. J. Cook, P. Jarrett, J. A. Cameron, S. J. Huang, and J. P. Bell, "Fungal degradation of polycaprolactones," Journal of Applied Polymer Science, vol. 28, no. 1, pp. 327-334, 1983.

[126] C. V. Benedict, J. A. Cameron, and S. J. Huang, "Polycaprolactone degradation by mixed and pure cultures of bacteria and a yeast," Journal of Applied Polymer Science, vol. 28, no. 1, pp. 335-342, 1983.

[127] W. J. Cook, J. A. Cameron, J. P. Bell, and S. J. Huang, "Scanning electron microscopic visualization of biodegradation of polycaprolactones by fungi," Journal of Polymer Science, Polymer Letters Edition, vol. 19, no. 4, pp. 159-165, 1981.

[128] H. Jaffe, P. A. Giang, D. K. Hayes, J. A. Miller, and B. H. Stroud, "Implantable systems for delivery of insect growth regulators to livestock. II," in Control. Release Pestic. Pharm, D. H. Lewis, Ed., pp. 303-310, Springer US, Boston, MA, 1981.

[129] C. G. Pitt, F. I. Chasalow, Y. M. Hibionada, D. M. Klimas, and A. Schindler, "Aliphatic polyesters. I. The degradation of poly( $\epsilon$-caprolactone)in vivo," Journal of Applied Polymer Science, vol. 26, no. 11, pp. 3779-3787, 1981.

[130] C. G. Pitt, M. M. Gratzl, A. R. Jeffcoat, R. Zweidinger, and A. Schindler, "Sustained drug delivery systems II: factors affecting release rates from poly ( $\varepsilon$-caprolactone) and related biodegradable polyesters," Journal of Pharmaceutical Sciences, vol. 68, no. 12, pp. 1534-1538, 1979.

[131] T. Tokiwa and Y. Suzuki, "Hydrolysis of polyesters by lipases," Nature, vol. 270, no. 5632, pp. 76-78, 1977.

[132] A. Kowalski, A. Duda, and S. Penczek, "Kinetics and mechanism of cyclic esters polymerization initiated with tin (II) octoate. 3. Polymerization of L, L-dilactide," Macromolecular Rapid Communications, vol. 19, pp. 567-572, 2000.

[133] M. Petitti, A. A. Barresi, and M. Vanni, "Controlled release of vancomycin from PCL microcapsules for an ophthalmic application," Chemical Engineering Research and Design, vol. 87, no. 6, pp. 859-866, 2009.

[134] A. Cesari, M. V. Loureiro, M. Vale, E. I. Yslas, M. Dardanelli, and A. C. Marques, "Polycaprolactone microcapsules containing citric acid and naringin for plant growth and sustainable agriculture: physico-chemical properties and release behavior," Science of the Total Environment, vol. 703, article 135548, 2020.

[135] M. Gilbert, "Aliphatic polyamides," in Brydson's Plast. Mater. Eighth Ed., vol. 5, pp. 487-511, Butterworth-Heinemann, 2017.

[136] S. Negoro, T. Taniguchi, M. Kanaoka, H. Kimura, and H. Okada, "Plasmid-determined enzymatic degradation of nylon oligomers," Journal of Bacteriology, vol. 155, no. 1, pp. 22-31, 1983.

[137] S. Kinoshita, T. Terada, T. Taniguchi et al., "Purification and characterization of 6-aminohexanoic-acid-oligomer hydrolase of flavobacterium sp. KI72," European Journal of Biochemistry, vol. 116, no. 3, pp. 547-551, 1981.

[138] S. Kinoshita, S. Kageyama, K. Iba, Y. Yamada, and H. Okada, "Utilization of a cyclic dimer and linear oligomers of $\varepsilon$-aminocaproic acid byAchrornobacter guttatusKI 72," Agricultural and Biological Chemistry, vol. 39, no. 6, pp. 12191223, 1975.

[139] S. Kinoshita, S. Negoro, M. Muramatsu, V. S. Bisaria, S. Sawada, and H. Okada, "6-Aminohexanoic acid cyclic dimer hydrolase. A new cyclic amide hydrolase produced by Acromobacter guttatus KI72," European Journal of Biochemistry, vol. 80, no. 2, pp. 489-495, 1977.

[140] T. Fukumura, "Two bacterial enzymes hydrolyzing oligomers of 6-aminocaproic acid," Journal of Biochemistry, vol. 59, no. 6, pp. 537-544, 1966.

[141] S. J. Huang, M. Bitritto, K. W. Leong, J. Pavlisko, M. Roby, and J. R. Knox, "The Effects of Some Structural Variations on the Biodegradability of Step-Growth Polymers," in Stab. Degrad. Polym, pp. 17-205, American Chemical Society, 1978. 
[142] H. C. Wang, J. M. Grolman, A. Rizvi, G. S. Hisao, C. M. Rienstra, and S. C. Zimmerman, "PH-triggered release from polyamide microcapsules prepared by interfacial polymerization of a simple diester monomer," ACS Macro Letters, vol. 6, no. 3, pp. 321-325, 2017.

[143] B. Tylkowski, M. Pregowska, E. Jamowska, R. Garcia-Valls, and M. Giamberini, "Preparation of a new lightly crosslinked liquid crystalline polyamide by interfacial polymerization. Application to the obtainment of microcapsules with photo-triggered release," European Polymer Journal, vol. 45, no. 5, pp. 1420-1432, 2009.

[144] M. Rogulska, A. Kultys, and W. Podkościelny, "Studies on thermoplastic polyurethanes based on new diphenylethanederivative diols. II. Synthesis and characterization of segmented polyurethanes from HDI and MDI," European Polymer Journal, vol. 43, no. 4, pp. 1402-1414, 2007.

[145] R. T. Darby and A. M. Kaplan, "Fungal susceptibility of polyurethanes," Applied Microbiology, vol. 16, no. 6, pp. 900-905, 1968.

[146] S. A. Guelcher, "Biodegradable polyurethanes: synthesis and applications in regenerative medicine," Tissue Engineering. Part B, Reviews, vol. 14, no. 1, pp. 3-17, 2008.

[147] S. J. Huang, C. Macri, M. Roby, C. Benedict, and J. A. Cameron, Biodegradation of Polyurethanes Derived from Polycaprolactonediols, American Chemical Society, 1981.

[148] P. Kardar, "Preparation of polyurethane microcapsules with different polyols component for encapsulation of isophorone diisocyanate healing agent," Progress in Organic Coatings, vol. 89, pp. 271-276, 2015.

[149] X. Liu, H. Zheng, G. Li et al., "Fabrication of polyurethane microcapsules with different shapes and their influence on cellular internalization," Colloids and Surfaces. B, Biointerfaces, vol. 158, pp. 675-681, 2017.

[150] Y. Ma, Z. Li, H. Wang, and H. Li, "Synthesis and optimization of polyurethane microcapsules containing [BMIm]PF6 ionic liquid lubricant," Journal of Colloid and Interface Science, vol. 534, pp. 469-479, 2019.

[151] R. He, J. Wang, X. Wang, W. Li, and X. Zhang, "Fabrication and characterization of core-shell novel PU microcapsule using TDI trimer for release system," Colloids and Surfaces A: Physicochemical and Engineering Aspects, vol. 550, pp. 138-144, 2018.

[152] A. D. Ribeiro, J. Marques, M. Forte et al., "Microencapsulation of citronella oil for solar-activated controlled release as an insect repellent," Applied Materials Today, vol. 5, pp. 9097, 2016.

[153] J. E. Bucher and W. C. Slade, "The anhydrides of isophthalic and terephthalic acids," Journal of the American Chemical Society, vol. 31, no. 12, pp. 1319-1321, 1909.

[154] R. Leong, K. Simonte, and V. Langer, "Synthesis of polyanhydrides: melt-polycondensation, dehydrochlorination, and dehydrative coupling," Macromolecules, vol. 20, no. 4, pp. 705-712, 1987.

[155] J. A. Tamada and R. Langer, "Erosion kinetics of hydrolytically degradable polymers," Proceedings of the National Academy of Sciences of the United States of America, vol. 90, no. 2, pp. 552-556, 1993.

[156] D. S. Katti, S. Lakshmi, R. Langer, and C. T. Laurencin, "Toxicity, biodegradation and elimination of polyanhydrides," Advanced Drug Delivery Reviews, vol. 54, no. 7, pp. 933961, 2002.
[157] R. Chasin, M. Lewis, and D. Langer, "Polyanhydrides for controlled drug delivery," Biopharm Manufact, vol. 1, pp. 33-46, 1988.

[158] N. Kumar, R. S. Langer, and A. J. Domb, "Polyanhydrides: an overview," Advanced Drug Delivery Reviews, vol. 54, no. 7, pp. 889-910, 2002.

[159] K. W. Leong, J. Kost, E. Mathiowitz, and R. Langer, "Polyanhydrides for controlled release of bioactive agents," Biomaterials, vol. 7, no. 5, pp. 364-371, 1986.

[160] J. Tamada and R. Langer, "The development of polyanhydrides for drug delivery applications," Journal of Biomaterials Science Polymer Edition, vol. 3, no. 4, pp. 315-353, 1992.

[161] J. G. Werner, S. Nawar, A. A. Solovev, and D. A. Weitz, "Hydrogel microcapsules with dynamic $\mathrm{pH}$-responsive properties from methacrylic anhydride," Macromolecules, vol. 51, no. 15, pp. 5798-5805, 2018.

[162] D. C. Casey and J. P. Manly, "No Title," in Proc. 3rd Int. Biodegrad. Symp. Appl. Sci. Publ, p. 819, New York, 1976.

[163] Y. Watanabe, M. Morita, N. Hamada, and Y. Tsujisaka, "Formation of hydrogen peroxide by a polyvinyl alcohol degrading enzyme," Agricultural and Biological Chemistry, vol. 39, no. 12, pp. 2447-2448, 1975.

[164] M. Morita and Y. Watanabe, "A secondary alcohol oxidase: a component of a polyvinyl alcohol degrading enzyme preparation," Agricultural and Biological Chemistry, vol. 41, no. 8, pp. 1535-1537, 1977.

[165] Y. Watanabe, N. Hamada, M. Morita, and Y. Tsujisaka, "Purification and properties of a polyvinyl alcoholdegrading enzyme produced by a strain of pseudomonas," Archives of Biochemistry and Biophysics, vol. 174, no. 2, pp. 575-581, 1976.

[166] K. Fukanage, F. Sumina, S. Neda, K. Takemasa, and T. Tachibana, jpn kokai, vol. 7, no. 794, p. 471, 1977.

[167] F. W. Harris and L. K. Post, "Synthesis of polymers containing pendent herbicide substituents," in Polym. Prepr. Div. Polym. Chem., pp. 622-627, Am. Chem. Soc, 1975.

[168] L. K. Harris and F. W. Post, "Synthesis and polymerization of the vinyl and acryloyloxyethyl esters of 2,4-dichlorophenoxyacetic acid and 2-(2,4,5-trichlorophenoxy) propionic acid," Journal of Polymer Science Part C: Polymer Letters, vol. 13, no. 4, pp. 225-229, 1975.

[169] A. Galbiati, B. M. Della Rocca, C. Tabolacci, S. Beninati, A. Desideri, and G. Paradossi, "PVA engineered microcapsules for targeted delivery of camptothecin to HeLa cells," Materials Science and Engineering: C, vol. 31, no. 8, pp. 1653-1659, 2011.

[170] J. Kozlowska and A. Kaczmarkiewicz, "Collagen matrices containing poly(vinyl alcohol) microcapsules with retinyl palmitate - structure, stability, mechanical and swelling properties," Polymer Degradation and Stability, vol. 161, pp. 108-113, 2019.

[171] E. Piacentini, F. Bazzarelli, T. Poerio et al., "Encapsulation of water-soluble drugs in poly (vinyl alcohol) (PVA)- microparticles via membrane emulsification: influence of process and formulation parameters on structural and functional properties," Materials Today Communications, vol. 24, article 100967, 2020.

[172] J. E. Potts, "Plastics, environmentally degradable," in KirkOthmer Encycl. Chem. Technol. Suppl. Vol., M. Grayson, Ed., p. 626, Wiley-Interscience, New York, 1985. 
[173] B. Baum and R. D. Deanin, "Controlled UV degradation in plastics," Polymer - Plastics Technology and Engineering, vol. 2, no. 1, pp. 1-28, 1973.

[174] A. F. Hegyeli, "Use of organ cultures to evaluate biodegradation of polymer implant materials," Journal of Biomedical Materials Research, vol. 7, no. 2, pp. 205-214, 1973.

[175] W. R. Vezin and A. T. Florence, "In vitro heterogeneous degradation of poly(n-alkyl ?-cyanoacrylates)," Journal of Biomedical Materials Research, vol. 14, no. 2, pp. 93-106, 1980.

[176] F. Leonard, R. K. Kulkarni, G. Brandes, J. Nelson, and J. J. Cameron, "Synthesis and degradation of poly (alkyl $\alpha$-cyanoacrylates)," Journal of Applied Polymer Science, vol. 10, no. 2, pp. 259-272, 1966.

[177] D. Wichterle and O. Lim, "Hydrophilic gels for biological use," Nature, vol. 185, no. 4706, pp. 117-118, 1960.

[178] J. Kopecek and L. Sprincl, "Relationship between the structure and biocompatibility of hydrophilic gels," Polimery $w$ Medycynie, vol. 4, no. 2, pp. 109-117, 1974.

[179] H. Bartulin, J. Przybylski, M. Ringsdorf, and H. Ritter, "Pharmacologically active polymers, 6," Macromolecular Chemistry and Physics, vol. 175, pp. 1007-1010, 1974.

[180] H. Batz, H. G. Ringsdorf, and H. Ritter, "Pharmacologically active polymers, 7," Makromolekulare Chemie, vol. 175, no. 8, pp. 2229-2239, 1974.

[181] Y. Liu, Y. Sun, L. Sun, and Y. Wang, "In vitro and in vivo study of sodium polyacrylate grafted alginate as microcapsule matrix for live probiotic delivery," Journal of Functional Foods, vol. 24, pp. 429-437, 2016.

[182] Y. Zhang, J. Song, and H. Chen, "Preparation of polyacrylate/ paraffin microcapsules and its application in prolonged release of fragrance," Journal of Applied Polymer Science, vol. 133 , no. 42 , pp. $1-7,2016$. 